\title{
Defects and Correction Theories of Electromagnetics
}

\author{
Kexin $\mathrm{Yao}^{1}$ \\ ${ }^{1}$ Instiute of Mechanical Engineering of Shaanxi Province, Xian Metering Institution, Xian, P.R.China \\ Correspondence: Kexin Yao, Instiute of Mechanical Engineering of Shaanxi Province, Xian Metering Institution, \\ Xian, P.R.China. Tel: 86-134-8462-6424. E-mail: yayydwpq@163.com
}

Received: April 27, 2016

Accepted: May 15, 2016

Online Published: July 29, 2016

doi:10.5539/apr.v8n3p154

URL: http://dx.doi.org/10.5539/apr.v8n3p154

\begin{abstract}
Experiments show that, there is the electrostatic field around the permanent magnet; since the electromagnetics can not explain this phenomenon, it can be concluded that there are some defects in electromagnetics. This paper makes an analysis of the defects of electromagnetics from fourteen aspects. It is noted that, the basic defect of electromagnetics is that there is no explanation of any inherent causes and physical processes of electromagnetic induction, displacement current, Lorentz force and other surface phenomena. Moreover, it may also lead us to make incorrect inferences in the theoretical analysis of electromagnetics, e.g. the same direction of action and reaction, infinitely high kinematic velocity of magnetic field, etc. It can be seen from analysis of all electromagnetic phenomena that, all the electromagnetic phenomena will be inevitably accompanied by an electron motion; and the electron motion is bound to take effect through an electric field; therefore, the analysis of motion in an electric field is the basis for analysis of all electromagnetic phenomena. This paper proposes seven rules for analysis of motion in an electric field, analyzes the uniform motion and variable motion in an electric field on the basis of such rules, clarifies the inherent causes of Lorentz force, theoretically qualitatively and quantitatively demonstrates why there exists the electrostatic field at some locations around the permanent magnet, and considers that all the magnetic phenomena are resulted from the contraction of electric field in the direction of motion, which is derived from the natural law of constant velocity of light, and therefore all the electromagnetic phenomena is rooted in the constant velocity of light. Through the analysis of variable motion in an electric field, this paper proves the law of displacement current and electromagnetic induction, clarifies the physical processes of such phenomena, deduces the law of total current, explains why electric field strength and magnetic field strength of electromagnetic wave must be in same phase and why a plane wave has the electric and magnetic fields only with a certain length but no source, and considers that neither fast nor slow propagation velocity of electromagnetic wave is good and that the actual velocity of light is the ideal velocity of light.
\end{abstract}

Keywords : electric field, magnetic field, permanent magnet, special relativity, electromagnetic induction, displacement current, Lorentz force, electromagnetic wave, constant speed of light

\section{Introduction}

For 200 years or more, we have considered the electromagnetics as a truth, regardless of its defects and errors. However, if we carefully analyze the essential principle of electromagnetics and some inferences in a larger range, we can find that there are some obvious defects in electromagnetics. First of all, we can see that, the basic principle of electromagnetics (e.g. electromagnetic induction, displacement current, Lorentz force and the like) shall be considered as the law concluded from the superficial phenomena; it neither further clarifies any inherent causes of electromagnetic phenomena, nor indicates the basic factors for phenomena, as if the moon waxing and waning law was summarized only in ancient times, but its causes of formation could not be explained. Today, we have already explained why the moon waxes and wanes. However, it has not yet explained about the causes of electromagnetic induction, displacement current, Lorentz force and other electromagnetic phenomena which obviously is a basic defect of electromagnetics. For another example, it is indicated in the Biot-Savart law of electromagnetics that, the moving electric charge (or current-carrying conductor) will generate a static magnetic field, while there is still an electron motion inside all the current-carrying objects in the universe, including the current-carrying conductor, magnet and other objects being in motion. The magnetic field generated by various electron motions shall be still 
expressed by the Biot-Savart law, i.e. such different moving electrons will generate a static magnetic field without (any) exception. That is to say, there is no a magnetic field being in motion in the universe. Obviously, this conclusion contradicts the judgment on electromagnetics that the magnetic field will be accompanied by the magnetic source in common motion. As another example, so far, the electromagnetics is unable to make a scientifically rational explanation for high-velocity moving charged particles deflecting in a magnetic field; any existing explanation is as follows: It is assumed that there is no time-to-time conversion, while there is mass conversion only; or, it is assumed that there is no mass conversion, while there is time-to-time conversion only. It is clear that such one-sided explanation is unscientific. More importantly, experiments show that, there exists the electrostatic field in some parts of uncharged permanent magnet, which proves that the Coulomb's law and Gauss theorem of electromagnetics shall not apply to all the areas. Also, it is demonstrated with certainty that, there are some defects in electromagnetics.

Also, it is affirmed that there are some defects in electromagnetics. Therefore, it is bound to look for ways to eliminate such defects, which requires an in-depth analysis of the inherent causes leading to all electromagnetic phenomena, the summary of the basic factors for all electromagnetic phenomena, the establishment of some new analysis methods to correct the defects of electromagnetics.

\section{Defects and Theoretical Fallacies of Electromagnetics}

In this paper, I'll first briefly introduce the experiment to determine whether there exists an electrostatic field around the permanent magnet in order to confirm the defects in electromagnetics. In 2004, I conducted an experiment to determine whether an electrostatic field existed around the permanent magnet. Basic principle: If there was an electrostatic field near a certain part of permanent magnet, the charged body would move under the action of electric field force. The first condition for this experiment was to eliminate the electrostatic induction of charged body on the permanent magnet. Or else, in any case, the charged body would be attracted by the permanent magnet. My determined permanent magnet was a cube NdFeB magnet (length of side: $20 \mathrm{~mm}$; surface magnetic induction intensity: $0.8 \mathrm{~T}$ or so). The determined results showed that, there was no electrostatic field near the center of $\mathrm{N} \& \mathrm{~S}$ surfaces and four sides of cube magnet; there was the negative electrostatic field near the four sides of N \& S-pole surfaces; and there was the positive electrostatic field near the four sides of permanent magnet. The detailed experimental methods and determined results should be shown in Section 4.3.2 below.

The above experimental results show that, in this case, the Coulomb's law of electromagnetics is not valid; and the electric flux of sealing surface surrounding this permanent magnet is not equivalent to zero, namely, the Gauss theorem is not valid too. In other words, the basic principle of electromagnetics shall not be consistent with the experimental results; so it can be confirmed that there are some defects in electromagnetics. This paper will make an explanation of principle defects and theoretically-deduced fallacies of electromagnetics from fourteen aspects below.

\subsection{Current-Generating Magnetic Field is an Unscientific Viewpoint of Action at a Distance.}

It is indicated in electromagnetics that, the moving electric charge or current-carrying conductor will generate a magnetic field around them and the specific magnetic field condition is calculated by the Biot-Savart law. According to the principle, there is no magnetic field at a certain point in space. When there is the moving electric charge or current-carrying conductor somewhere beyond this point, there will be the magnetic field; that is to say, the current can generate a magnetic field at any location across any distance. Obviously, this is a viewpoint of action at a distance. It is well known that, the viewpoint of action at a distance is an unscientific point of view which should be denied. The scientific point of view should indicate why the current will generate a magnetic field around it, and what are the inherent causes of current-generating magnetic field. Obviously, the electromagnetics has made no explanation for this.

\subsection{The Displacement Current Formula can not Illustrate the Specific Distribution of Induced Magnetic Field}

It is indicated in electromagnetics that the changing electric field will form the displacement current. Maxwell Definition: The displacement current density at a point of electric field shall be equivalent to the change rate of electric displacement vector with respect to time at the said point. Like the conduction current, the displacement current will also generate a magnetic field. In a vacuum, the relation between displacement current and its generating magnetic field can be expressed in the following formula: 


$$
\oint_{l} \mathrm{H} \cdot d l=\int_{s} \varepsilon_{0} \frac{d \mathrm{E}}{d t} \cdot d \mathrm{~S}
$$

As can be seen, the displacement current has not yet given an explanation for inherent causes of generating a magnetic field at a certain point in space and we can not calculate the specific conditions of magnetic field (magnitude and direction) at a certain point in space.

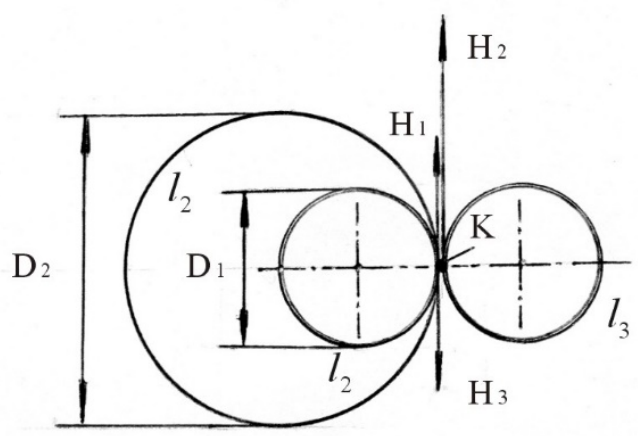

(a)

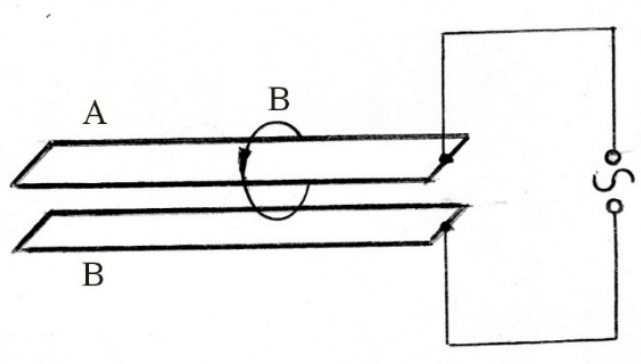

(b)

Figure 1.

There is a large uniformly-distributed changing electric field on the vertical principal plane (as shown in Figure 1 (a)). The changing electric field is the displacement current generating magnetic field in space. Based on the above-mentioned formula of the relation between the magnetic field and the displacement current, if we select a circular loop with diameter $\mathrm{D}$, and it is assumed that $\mathrm{H}$ is uniformly distributed along the circular loop, then $\pi D H=\pi D^{2} \varepsilon_{0} d E / d t$, i.e. $H=D \varepsilon_{0} d E / d t$, which can lead to the conclusion that the magnetic field strength is proportional to the diameter of our selected loop, like in the picture, if $D_{2}=2 D_{1}$, then $H_{2}=2 H_{1}$ and since $H$ at a certain point is unique, the calculated result $\left(H_{2}=2 H_{1}\right)$ is clearly wrong. If we also select a loop $l_{3}$ with diameter $D_{1}$, then there must be $H_{3}$ and $H_{1}$ being of the same magnitude and the opposite direction at the same point $\mathrm{K}$ (as shown in Figure 1 (a)). Since $H$ is unique, so the conclusion is clearly wrong that there may be $H$ in two opposite directions at the same point. The above analysis shows that, according to the formula of relation between the displacement current and the magnetic field, it is unlikely to directly estimate the conditions of magnetic field at a point in space like the Biot-Savart law. Can we deduce the conditions of magnetic field at a point in space through the overall changing electric field based on the displacement current? The analysis shows that, for the actual changing electric field, it is unlikely to deduce the conditions of magnetic field at a point in space based on the displacement current. As shown in Fig 1 (b), after the right terminals of two elongated flat plates $\mathrm{A} \& \mathrm{~B}$ are connected to the $\mathrm{AC}$ power via a connecting lead, a changing electric field will be generated between $\mathrm{A}$ and meanwhile a changing magnetic field will also be generated accordingly. Obviously, the power supply current is coming in and out from the right terminals of A and B. It is observed that the current of A and B will be inevitably reduced gradually from right to left. The current at the leftmost end will always be zero. According to the Biot-Savart law, it is observed that, the magnetic field strength between A and B will be inevitably reduced gradually from right to left. Obviously, it is impossible to accurately calculate the conditions of magnetic field between A and B. The above analysis shows that it is not aimed to deny the displacement current, but it points out that the electromagnetics has not clarified the inherent causes for generating the displacement current, which therefore leads to people's improper use of the concept of displacement current.

\subsection{The Electromagnetic Induction Law can not Explain how the Induced Electric Field is Generated}

The electromagnetic induction law of electromagnetics indicates that the electric field will be induced by the changing magnetic field. Why can the electric field be generated by the changing magnetic field? The electromagnetics has made no specific explanation for this. People can only think that this is the inevitable law, as if the ancient people thought that the moon waxing and waning law was an inexorable law. So, the law of electromagnetic induction has become very mysterious. In particular, it indicates that the induced electric field not 
only exists in the changing magnetic field, but also in the unchanging magnetic field. For example, after the transformer is connected to the AC power, the iron core of transformer will generate an alternating magnetic field, while there is basically no alternating magnetic field anywhere outside the iron core of transformer. However, there is an electric potential with the same magnitude on any closed loop surrounding the iron core of transformer. This means that there is the induced electric field in the unchanging magnetic field outside the iron core of transformer. In other words, the changing magnetic field may generate an electric field across any distance at any point in space. This is an unscientific viewpoint of action at a distance, for which the fundamental reason obviously is the electromagnetics did not clarify the inherent causes and physical processes of electromagnetic induction.

\subsection{Lorentz Force is not a Universally-Correct Formula}

It is noted in electromagnetics that, when a charged particle passes through the magnetic field, the particle will be subject to an applied force perpendicular to the velocity of particle motion (called the Lorentz force). Lorentz summarized some of the existing experimental results, and pointed out that the Lorentz force was proportional to the velocity of particle motion and the magnetic induction intensity. Obviously, Lorentz force is only a summary of the experimental phenomena without the explanation of inherent reasons of generating the force. His experimental results neither contain the motion of high-velocity charged particles in a magnetic field, nor include the motion of charged particles in a non-uniform magnetic field. Therefore, his experiments shall be considered as one-sided experiments. Since people can neither understand the inherent reasons of generating the force nor fully understand all the force-bearing conditions of charged particles, which makes the Lorentz force only a formula within a limited scope of application. For example, the application of Lorentz force cannot help to scientifically explain the deflection results of high-velocity moving charged particles in a magnetic field, nor reasonably judge the force bearing conditions of charged particles in a non-uniform magnetic field (see Section 2.6).

\subsection{Discussion of Electromagnetic Wave is Lack of the Physical Illustration}

For the analysis of electromagnetic wave, apart from some mathematical deductions, the electromagnetics has not basically clarified the physical processes and actual conditions of electromagnetic wave. In many books, the changing magnetic field is generated by the changing electric field, while the changing electric field is generated by the changing magnetic field, and so on, so that the electromagnetic wave is formed. It can be seen from the analysis that this explanation is certainly wrong, because the changing magnetic field is generated by the changing electric field, or the changing electric field is generated by the changing magnetic field; the result, of course, is that, there is a phase difference $\left(90^{\circ}\right)$ between the electric field intensity vector and magnetic field intensity vector of electromagnetic wave, but there is no a phase difference $\left(90^{\circ}\right)$ between the electric field intensity vector and magnetic field intensity vector of actual electromagnetic wave, both of which are always in phase. Apart from the mathematical derivation, the electromagnetics can not make an explanation for such a result from physical significance. For another example, if the directional transmission plane electromagnetic wave can be expressed by the electric line of force and the magnetic line of force, there is the electric line of force with a certain length (electric field intensity) in the longitudinal direction of electromagnetic wave, but there is the magnetic line of force with a certain length (magnetic field intensity) in the transverse direction of electromagnetic wave. As we all know, the electric line of force can only be a radial line (e.g. point-charged electric field) or a closed line (e.g. changing magnetic field-induced electric field), while there can not be only the electric line of force with a certain length; the magnetic line of force can only be a closed line, while there can not be only the magnetic line of force with a certain length. Obviously, the electromagnetics can not make a scientifically rational explanation for such an electric line of force and magnetic line of force in terms of physics.

\subsection{Both Action and Reaction May have the Same Direction but the Different Magnitude.}

As shown in Figure 2, the isolated negative charge $-Q$ (such as high-voltage discharge charge) is in motion along the central line KJ of current-carrying coil at velocity V. According to the Biot-Savart law, the direction of magnetic field generated by $-Q$ above the central line $\mathrm{KJ}$ is a direction of traversing through the principal plane (expressed by small $\odot$ in Figure 2); the direction of magnetic field generated by $-Q$ below the central line $\mathrm{KJ}$ is a direction of penetrating into the principal plane (expressed by small $\otimes$ ). According to the right-hand screw rule, the direction of magnetic field generated by current-carrying coil $\mathrm{ABCD}$ inside a coil is a direction of traversing through the principal plane (expressed by large $\odot$ ); the direction of magnetic field generated by current-carrying coil ABCD outside a coil is a direction of penetrating into the principal plane (expressed by large $\otimes$ ). Obviously, 
according to the Lorentz force formula, the direction $\boldsymbol{F}_{Q}$ of Lorentz force applied to $-Q$ is in the direction of $\boldsymbol{Y}_{o}$ (see Figure 2). $\boldsymbol{Y}_{o}$ represents the unit vector in the direction of $\boldsymbol{Y}$.

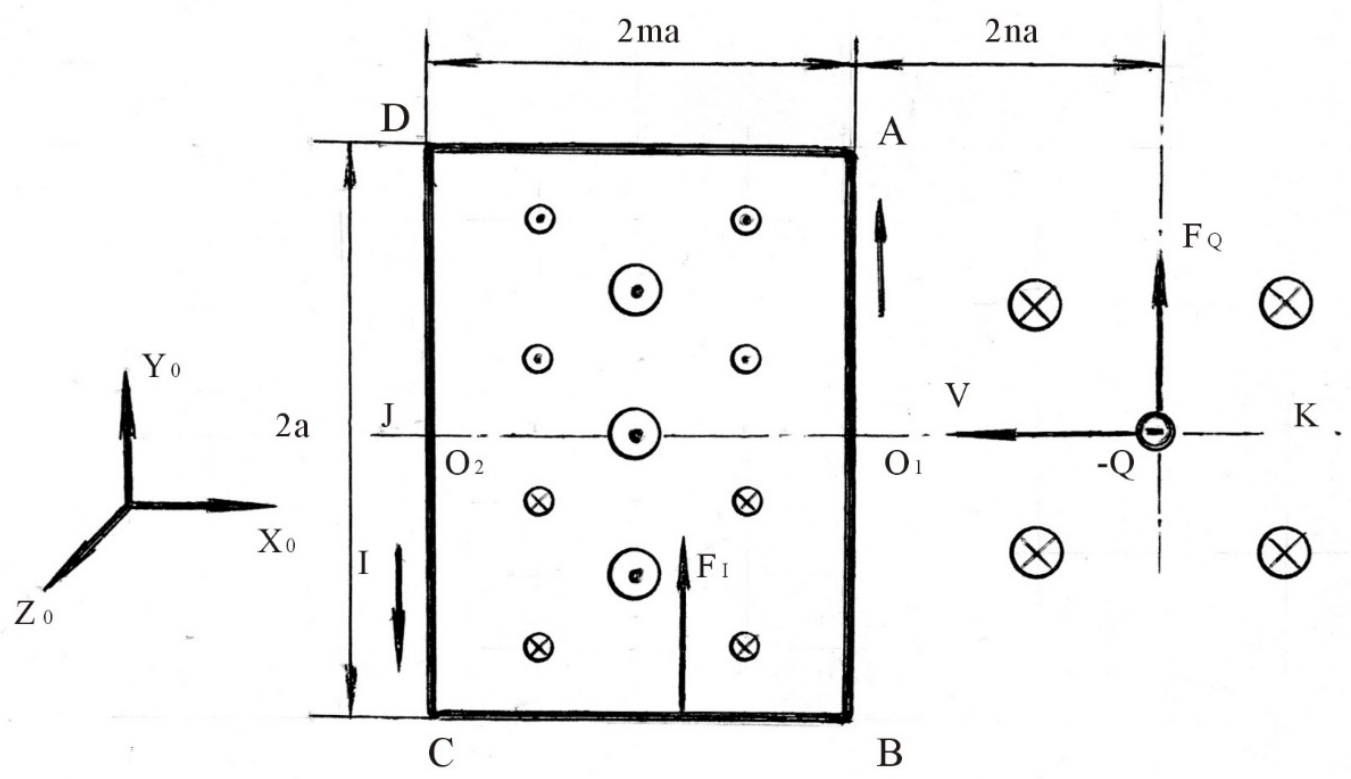

Figure 2. Motion of electric charge outside the coil

Let us analyze the force applied on the coil $\mathrm{ABCD}$ by the magnetic field generated by $-Q$. It can be seen from analysis that, the forces applied on the DA and BC sections of coil by the magnetic field generated by $-Q$ have the same magnitude and the same direction (DA and BC have the opposite direction of current and the opposite direction of magnetic field). The resultant force of both is expressed by $F_{1}$. The calculated value of $F_{1}$ shall be as follows (the derivation can be omitted):

$$
F_{1}=\frac{\mu_{O} I Q V}{\pi a}\left[\frac{m+n}{\sqrt{1+4(m+n)^{2}}}-\frac{n}{\sqrt{1+4 n^{2}}}\right]
$$

It can be seen from analysis that, as a result of any small section of current element on DA and BC current-carrying conductor, the interaction force between $-Q$ and $\mathrm{DA} \& \mathrm{BC}$ current-carrying conductor has the same magnitude and the opposite direction. Therefore, the total force applied on $-Q$ by $\mathrm{DA}$ and $\mathrm{BC}$ current-carrying conductor shall be bound to equal to $-F_{l}$, i.e. the interaction force between $-Q$ and $\mathrm{DA} \& \mathrm{BC}$ has the same magnitude and the opposite direction.

Let's analyze the force applied on $\mathrm{AB}$ and $\mathrm{BC}$ of coil by $-Q$ magnetic field. The force applied on $\mathrm{AB}$ by $-Q$ shall be shown in the Figure. $\mathrm{AO}_{1}$ (the upper half of $\mathrm{AB}$ ) and $\mathrm{O}_{1} \mathrm{~B}$ (the lower half of $\mathrm{AB}$ ) have the same length, the same current but the opposite direction of force applied by $-Q$ magnetic field. Therefore, the forces applied on $\mathrm{AO}_{1}$ and $\mathrm{O}_{1} \mathrm{~B}$ by $-Q$ magnetic field have the same magnitude and the opposite direction; the resultant force of zero. Likewise, the resultant force applied on $\mathrm{CD}$ by $-Q$ magnetic field must be zero. Therefore, our conclusion is that, the force applied on $\mathrm{ABCD}$ of coil by $-Q$ magnetic field can only include the resultant force applied on DA and $\mathrm{BC}$ by $-Q$ magnetic field (no force is applied on $\mathrm{AB}$ and $\mathrm{CD}$ ), i.e. $F_{I}$. Since $F_{I}$ is in the direction of $\boldsymbol{Y}_{0}$, and the Lorentz force $\boldsymbol{F}_{Q}$ applied on $-Q$ by current-carrying coil magnetic field is also in the direction of $\boldsymbol{Y}_{0}$, i.e. $F_{I}$ and $\boldsymbol{F}_{Q}$ have the same direction, thus we have drawn a strange conclusion, namely, the force applied on coil $\mathrm{ABCD}$ by $-Q$ magnetic field and the force applied on $-Q$ by coil $\mathrm{ABCD}$ magnetic field have the same direction, that is to say, both action and reaction have the same direction. 
The above analysis shows that, the force applied on current-carrying coil $\mathrm{AB}$ and $\mathrm{BC}$ by $-Q$ magnetic field shall be zero. The force applied on $-Q$ by $\mathrm{AB}$ and $\mathrm{CD}$ magnetic field is zero, isn't it? From the scoreboard derivation, the force applied on $-Q$ by $\mathrm{AB}$ magnetic field shall be as follows (the derivation can be omitted):

$$
\boldsymbol{F}_{Q}^{A B}=\frac{\mu_{o} \operatorname{IQV}}{2 \pi n a \sqrt{1+4 n^{2}}} \boldsymbol{Y}_{o}
$$

The force applied on $-Q$ by $C D$ magnetic field shall be as follows:

$$
F_{Q}^{c D}=\frac{\mu_{0} I Q V}{2 \pi(m+n) a \sqrt{1+4(m+n)^{2}}}\left(-Y_{\theta}\right)
$$

Obviously, $\boldsymbol{F}_{Q}^{A B}$ is much greater than $C$, i.e. the total force applied on $\operatorname{tg} \alpha$ by $\mathrm{AB}$ and $\mathrm{CD}$ magnetic field shall not be zero, that is to say, there is a different interaction between the $\mathrm{AB} \& \mathrm{CD}$ of current-carrying coil $\mathrm{ABCD}$ and $\alpha$.

Thus, our overall conclusion is that, the interaction force and reaction force between the current-carrying coil and the moving electric charge have the same direction but the different magnitude. Obviously, this conclusion violates the Newton's law of reaction, which should be a wrong conclusion.

\subsection{Disappearing Lorentz Force}

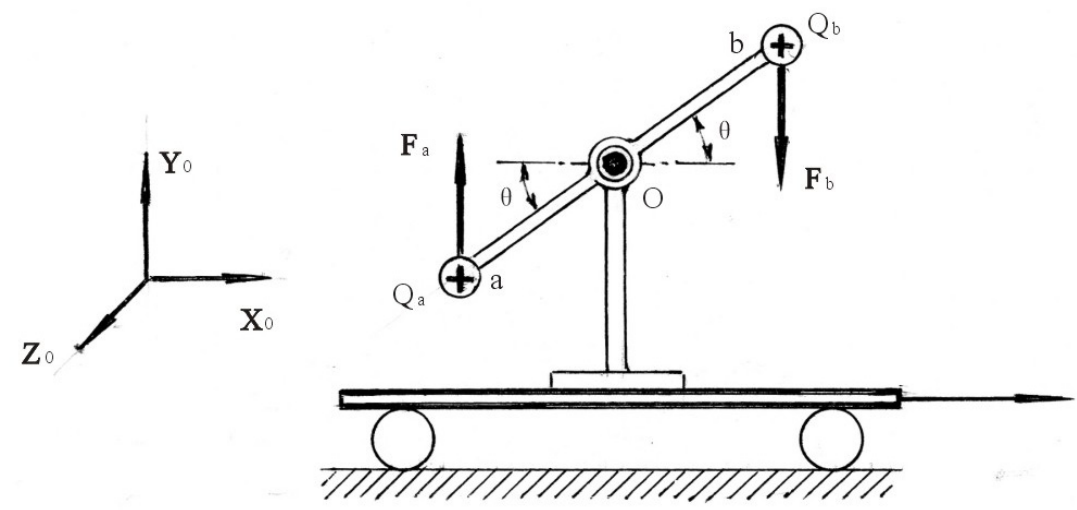

Figure 3. During the motion of trolley, A and B should be rotated

As shown in Figure 3, when a trolley is in motion at velocity $V$; an insulating $\operatorname{rod} a b$ with the length of $l$ can be rotated around the Point $\mathrm{O}$ of trolley; $a b$ have the positive charges with a certain quantity of electricity $Q_{a} \& Q_{b}$. The observer on the ground observes that, the positive charge $Q_{b}$ at $\mathrm{b}$ section is in motion at velocity $V$ together with the trolley, which is bound to generate a magnetic field. According to the Biot-Savart law, $Q_{b}$ at b section will generate the magnetic induction intensity at a section as follows:

$$
\boldsymbol{B}_{a}=\frac{\mu_{0}}{4 \pi} \frac{Q_{b} V}{l^{2}} \sin \theta\left(-Z_{0}\right) \quad Z_{0} \text { - Unit vector }
$$

Since $Q_{a}$ at a section is also in motion at velocity $V$, i.e. when $Q_{a}$ is passing through the magnetic field (magnetic induction intensity $B_{a}$ ) at velocity $V$, the observer necessarily deduces that the Lorentz force is applied on $Q_{a}$.

$$
F_{a}=V \times B_{a} Q_{a}=\frac{\mu_{0}}{4 \pi} \frac{Q_{a} Q_{b} V^{2}}{l^{2}} \sin \theta\left(Y_{0}\right)
$$

The direction of $F_{a}$ is a upward direction. Similarly, we can obtain the Lorentz force applied on $Q_{b}$ :

$$
F_{b}=\frac{\mu_{0}}{4 \pi} \frac{Q_{a} Q_{b} V^{2}}{l^{2}} \sin \theta\left(-Y_{0}\right)
$$

The direction of $F_{b}$ is a downward direction. Obviously, $F_{a}$ and $F_{b}$ have the same magnitude but the opposite direction, so as to form a couple. This couple will certainly allow $l$ to rotate around the Point O. However, relative to the trolley, $Q_{a}$ and $Q_{b}$ are static; for the trolley, $Q_{a}$ and $Q_{b}$ can not generate a magnetic field, on which the Lorentz force will not be applied; therefore, $l$ will not be rotated. Since $l$ of trolley is not rotated, the observer 
on the ground will certainly not see the rotation of $l$. In other words, the observer on the ground can deduce $Q_{a}$ and $Q_{b}$ based on the principle of electromagnetics; the Lorentz force shall be applied on $Q_{a}$ and $Q_{b}$, so that the result of the rotation of $l$ is not consistent with the facts, i.e. there are no Lorentz force $F_{a}$ and $F_{b}$ deduced by the observer.

\subsection{The Force and Magnetic Field will be Generated, and Changed with the Observer}

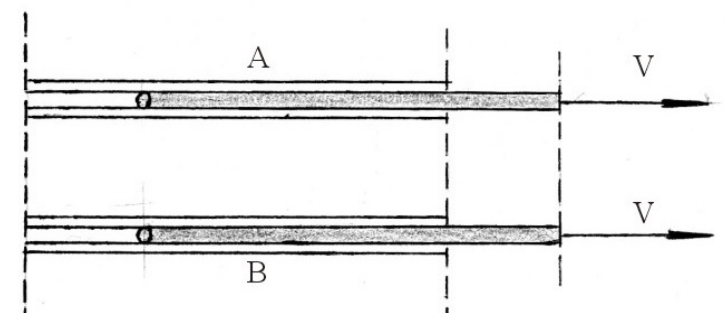

Figure 4. Changes of the force and magnetic field with the different observers

A and B can represent the two "infinitely long" parallel sections of current-carrying conductor (as shown in Figure 4). The current-carrying conductor shall be vividly considered as a pipeline in the middle of which the free electrons can flow; the pipe shell shall be considered as the protons which have the equivalent electric quantities as the free electrons. Based on the principle of electromagnetics, the person (who is static relative to the pipeline) considers that, the magnetic field will be generated by the free electrons moving at velocity $V$ in the pipeline $\mathrm{A}$ (or $\mathrm{B}$ ), while the Ampere's force will be applied on the free electrons moving at velocity $V$ in the pipeline $\mathrm{B}$ (or A) and passing through the magnetic field. Under the action of this force, the two pipelines will be attracted each other.

However, the person (who is static relative to the free electrons and moving at velocity $V$ relative to the pipeline) considers that, the magnetic field will be generated by the pipeline A (or B) containing the positively-charged protons and moving at velocity $-V$, while the Ampere's force will be applied on the pipeline B (or A) passing through the magnetic field at velocity $-V$. Under the action of this force being of the same magnitude as the above force, the two pipelines will be attracted each other.

Another person (who is moving at velocity $V / 2$ ) considers that, all the free electrons in the pipeline A and B are moving at velocity $V / 2$, generating the magnetic field, and acted upon by the Ampere's force. He also considers that, the proton pipelines $\mathrm{A}$ and $\mathrm{B}$ are moving at velocity $-V / 2$, generating the magnetic field, and acted upon by the Ampere's force. Obviously, it can be seen from the analysis by the person moving at velocity $V / 2$, the total magnetic field generated by $\mathrm{AB}$ current-carrying conductor has the same effect as the above two cases; the Ampere's force applied on the $\mathrm{AB}$ current-carrying conductor has the same effect as the above two cases.

As can be seen, although the total effects analyzed in the above three cases are identical, there are the completely-different opinions on the magnetic field generator and the acting point of force. We know that the truth should be unique. As shown in Figure 4, the magnetic field generator should be unique; the acting point of force should be unique; it is clearly unscientific that the different observers have their different opinions.

\subsection{The Biot-Savart Law Contradicts with the Principle of a Rotating Magnetic Field of Electromechanics}

It is noted in electromechanics that, after the stator of three-phase asynchronous motor is connected to the three-phase AC power source, a rotating magnetic field will be inevitably generated in the motor. This rotating magnetic field has exactly the same effect as the magnetic field generated when the stator with magnetic pole N\&S is rotated. The principle of rotating magnetic field is the theoretical basis for electromechanics. However, according to the Biot-Savart law of electromagnetics, after being connected to any type of power, all the magnet exciting coils of three-phase asynchronous motor stator can only generate the static magnetic field. For the AC power source, the static magnetic field is caused only by the changing intensity of the magnetic field definitely without the rotation at all.

All the practical results of electromagnetics have confirmed the existence of a rotating magnetic field. If we acknowledge the existence of a rotating magnetic field, it is necessary to deny the Biot-Savart law, isn't it? 


\subsection{We can not Determine Whether the Magnetic Field is Rotated.}

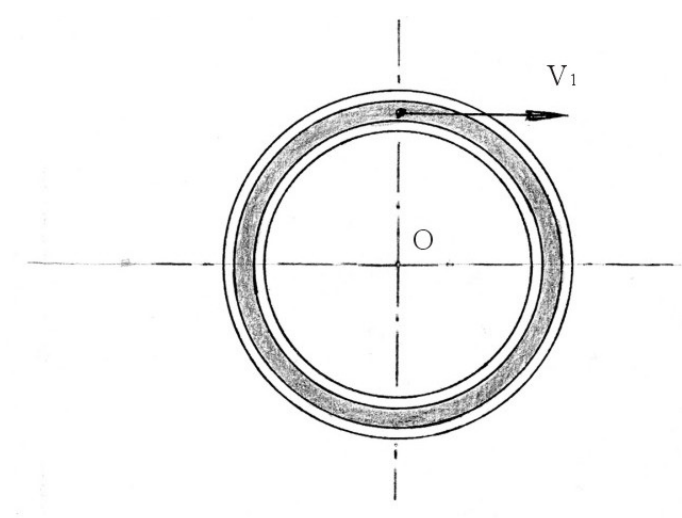

(a)

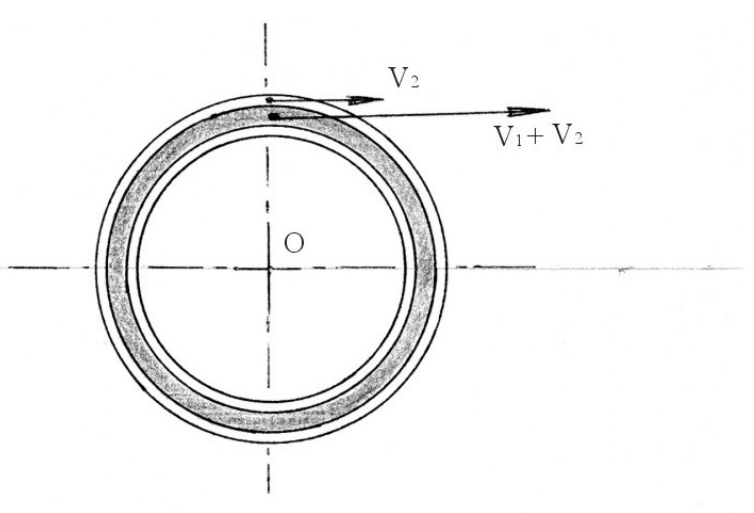

(b)

Figure 5. Circumferential current-carrying coil being rotated

For ease of explanation, the pipeline can still represent the current-carrying conductor. As shown in Figure 5a, after the static circumferential current-carrying coil is powered on, the free electrons in the pipeline will be in motion at velocity $V_{1}$. As shown in Figure 5b, after the current-carrying coil is rotated in the clockwise direction, the considerable coil protons (with the same electric quantities as free electrons) will be in motion at velocity $V_{2}$. Obviously, in this case, the velocity of motion of free electrons will become: $V_{1}+V_{2}$.

As can be seen, the magnetic field generated by the static current-carrying coil will be entirely generated by free electrons moving at velocity $V_{1}$, while the magnetic field generated by the current-carrying coil being rotated at linear velocity $V_{2}$ will be generated by protons moving at velocity $V_{2}$ and free electrons moving at velocity $V_{1}+V_{2}$. Since the direction of magnetic field generated by protons being in motion is opposite to the direction of magnetic field generated by electrons being in motion, and the magnetic field intensity is proportional to the velocity of electron or proton motion, the magnetic field generated by protons moving at velocity $V_{2}$ will inevitably offset the magnetic field generated by free electrons moving at velocity $V_{2}$ (as shown in $5 \mathrm{~b}$ ); the total effect of magnetic field generated by protons moving at velocity $V_{2}$ is identical to the total effect of magnetic field generated by free electrons moving at velocity $V_{1}$. Thus, we can draw the following conclusions: Regardless of whether the circumferential current-carrying coil is rotated or not, the magnetic field conditions are identical.

From another point of view, relative to the current-carrying coil, i.e. protons, the magnetic field generated by the circumferential current-carrying coil should be static. Thus, if the current-carrying coil is rotated, the magnetic field generated by the current-carrying coil will be inevitably rotated.

From the above analysis, we have drawn two conclusions: When the circumferential current-carrying coil is rotated, the magnetic field generated by the circumferential current-carrying coil will not be rotated; or the magnetic field generated by the circumferential current-carrying coil will be rotated together with the coil. Both analysis results are reasonable. There's no reason for us to absolutely affirm or negate them in the practice.

\subsection{The Magnetic Field Being in Motion Should be Static}

As shown in Figure 6, if the current-carrying coil abcd is in motion at velocity $V$, people intuitively think inevitably that, the magnetic field will be generated by the current in the coil abcd; when the coil is in motion, the magnetic field generated by the coil motion will naturally be in motion accordingly. Therefore, a magnetic field moving at velocity $V$ will inevitably be generated by the current-carrying coil.However, according to the Biot-Savart law, from the analysis, there are the different results. On account of the constant motion velocity of free electrons on wire segments, there is the same magnitude of motion velocity $V_{1}, V_{2}, V_{3} \& V_{4}$ of free electrons on wire segments ab, bc, cd \& da. Since all the free electrons in the coil are in motion at velocity $V$ along the coil, the actual resultant velocity of free electrons on segment ab is bound to $V_{a b}=V+V_{1}$; similarly, the actual resultant velocity of free electrons on segment bc, cd \& da is bound to $V_{b c}, V_{c d} \& V_{d a}$ (see the Figure). Clearly, $V_{a b}, V_{b c}, V_{c d}$ and $V_{d a}$ shall be 
the constant velocity motion. The magnetic field generated by the constant velocity motion of free electrons shall be inevitably consistent with the Biot-Savart law, i.e. it is bound to generate a static magnetic field. Therefore, the magnetic field generated by free electrons in the coil abcd will be inevitably a static magnetic field. Since the protons in the coil abcd (with the same electric quantities as free electrons) are in motion at constant velocity V, it is also bound to generate a static magnetic field according to the Biot-Savart law. Thus, we can draw the following conclusions: A static magnetic field will be generated by all the free electrons and protons in the coil abcd; the resultant total magnetic field must be a static magnetic field as well.

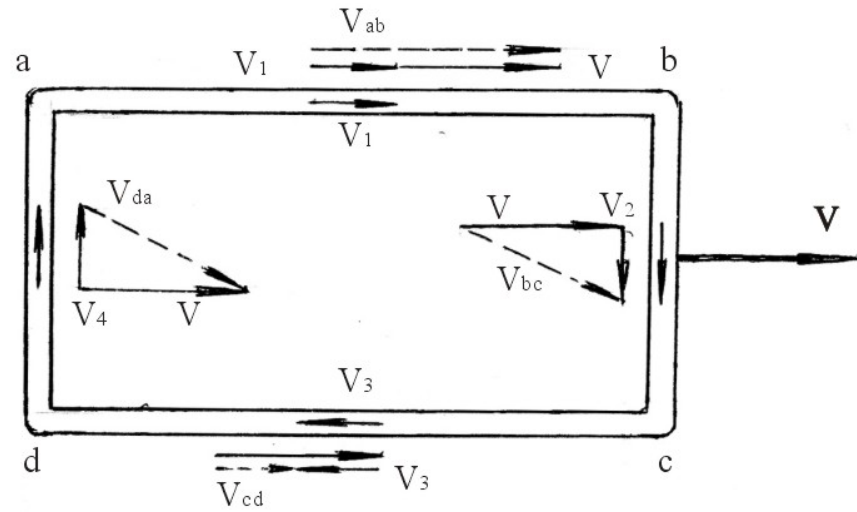

Figure 6. Static magnetic field generated by the current-carrying coil being in motion

\subsection{We can not Explain the Deflection Results of High-Velocity Charged Particles in a Magnetic Field}

It is noted in electromagnetics that, as a result of the action of Lorentz force, the charged particles will be inevitably deflected when passing through a magnetic field. The charged particles (the rest mass is set as $m$ ) will pass through a magnetic field at velocity $V$ along the direction $x$ within a period of time $t$. If $F$ represents the Lorentz force applied on charged particles, the deflection distance of charged particles in the direction y shall be as follows:

$$
Y=\frac{1}{2} a t^{2}=\frac{F}{2 m} t^{2}
$$

The Formula (a) can be derived under the constant conditions of direction $F$. Although there are some changes in velocity $V$ and direction $F(F \perp V)$ when the particle passes through the magnetic field, because of minimum time $t$, there are very small changes in $F$ and $V$, so that the error of $Y$ can be ignored.

Experiments show that, the Formula (a) holds good only if $V$ is very small. When $V$ is very large, the measured deflection distance of $\boldsymbol{Y}$ shall be as follows:

$$
Y=\frac{F}{2 m} t^{2} \sqrt{1-V^{2} / C^{2}}
$$

Where, $\mathrm{C}$ represents the beam of light. Formula (b) is a simplified formula. In the actual complete formula, the Lorentz force $B Q V$ ( $Q$ - charge carried by particle) must be replaced by $F$ in Formula (6), namely:

$$
Y=\frac{B Q V}{2 m} t^{2} \sqrt{1-V^{2} / C^{2}}
$$

Formula (c) is a formula applied to the deflection experiment. In fact, we don't know $m$ and $Q$ of particles in the experiment. Experiment is designed to obtain the charge-to-mass ratio $Q / m$ of particles. Obviously, Formula (c) is not consistent with Formula (a) $\left(Y=B Q V t^{2} / 2 m\right)$. In order to obtain the true Formula (c), the mass conversion equation of special relativity $m^{\prime}=m / \sqrt{1-V^{2} / C^{2}}$ is generally applied in electromagnetics; $m^{\prime}$ can replace $m$, and substituted into Formula (a), so as to obtain Formula (c).

Moreover, the deflection $\mathrm{Y}$ can be obtained by means of momentum change of charged particles, rather than the method for analysis of Lorentz force. In this method, the migration velocity of particles in the direction $Y$ shall be set as $u$ ). The mass of a particle can be expressed by the mass conversion equation of special 
relativity $m^{\prime}=m / \sqrt{1-V^{2} / C^{2}}$. The momentum of particles in the direction $Y$ can be obtained as $\left(P=m^{\prime} u=m u / \sqrt{1-V^{2} / C^{2}}\right)$ thus:

Since $_{F} \sqrt{1-V^{2} / C^{2}} / m$ is constant, thus:

$$
\begin{gathered}
\frac{d p}{d t}=\frac{m}{\sqrt{1-V^{2} / C^{2}}} \cdot \frac{d u}{d t}=F \\
\frac{d u}{d t}=\frac{F \sqrt{1-V^{2} / C^{2}}}{m}
\end{gathered}
$$

$$
Y=\int_{0}^{t} d t \int_{0}^{t} \frac{d u}{d t}=\frac{F}{2 m} t^{2} \sqrt{1-V^{2} / C^{2}}
$$

This deflection result is consistent with Formula (b).

It is noted that, the only basic reason for deflection of charged particles moving in a magnetic field is the action of Lorentz force. The basic method of physics is: To analyze the deflection of charged particles from the fundamental cause, i.e. Lorentz force. In the above method, the momentum changes of the particles is analyzed to obtain the acting force $d p / d t$, so as to further obtain the deflection $Y$. As can be seen, in this analysis method, there are no grounds for determining $(d p / d t)=B Q V$ and in the actual deflection experiment, the known quantity is $Q / m$ rather than $m$. Therefore, the deflection formula based on momentum changes of charged particles has no practical value owing to the unknown $m$.

The Berkeley physics describes a method for application of time-to-time conversion to deflection derivation. This method prescribes that, $\Delta t$ represents the particle displacement time; the particle mass is set as the constant rest mass $m ; \Delta Y$ represents the particle displacement in the time $\Delta t$ in the direction $Y$; thus the momentum $P=m \Delta Y / \Delta t$. ccording to the special relativity, the observer considers that, if $\Delta \tau=\Delta t \sqrt{1-V^{2} / C^{2}}$, then the momentu $P=m \Delta Y / \Delta \tau=m \Delta Y / \Delta t \sqrt{1-V^{2} / C^{2}}$. If the displacement velocity of particle in the $Y$ direction $u=\Delta Y / \Delta t$ is substituted into this formula, then $P=m u / \sqrt{1-V^{2} / C^{2}}$; this result is identical to $P$ in the analysis of change in momentum. According to the same derivation steps, we can naturally obtain the Formula (b). Obviously, this analysis method is an unscientific method, because it is used to firstly analyze the change of momentum and then the Lorentz force. Besides, this method also prescribes that the mass of particle is the constant rest mass, which is obviously wrong, because the mass change is an inexorable law of nature. Why can it prescribe that the mass isn't converted?

Let's look at the derivation of Formula (c): This formula is derived only through the mass conversion rather than time-to-time conversion, actually it specifies time isn't converted, which is obviously wrong. According to the Special relativity, the mass, time and length of moving object shall be converted at the same time. For the deflection of charged particles moving in a magnetic field, the length is not directly related, and thus the mass and time shall be converted simultaneously. If the mass conversion $m^{\prime}=m / \sqrt{1-V^{2} / C^{2}}$ and the time-to-time conversion $t^{\prime}=t / \sqrt{1-V^{2} / C^{2}}$ are substituted into Formula (a), then:

$$
Y=\frac{a}{2} t^{2}=\frac{B Q V}{2 m^{\prime}} t^{\prime 2}=\frac{B Q V}{2 m}\left(1-V^{2} / C^{2}\right)^{3 / 2}
$$

Formula (d), the result of the application of the Special relativity. is apparently inconsistent with the true deflection result of Formula (c), which shows that, under the conditions of mass conversion and time-to-time conversion, the electromagnetics can not explain the deflection of high-velocity charged particles in a magnetic field.

\subsection{Fundamental Difference between the Magnetic Field and the Real Substance}

It is noted in electromagnetics that, the magnetic field is one kind of substance, and generated by the electric current; that is to say, the magnetic field substance is generated by the electric current; there is a fundamental difference between the generated substance and the inherent real substance. For example, there is a charged body on a moving train; the people on the train observe that the charged body is static, and that there only exists an electrostatic field around the charged body. However, the people on the ground observe that the charged body is moving together with the train. The moving charged body is the electric current. According to the Biot-Savart law, 
a magnetic field will be inevitably generated by the electric current. This fact means that, about whether there exists a magnetic field around the same charged body, the different observers have different observation results. In other words, the existence of magnetic field substance is related to the observers. As we all know, the existence of all real substances (e.g. animals, plants, minerals, electrostatic field, gravitational field, etc.) will not be related to the observers, while the existence of magnetic field substance will be related to the observers, which means that the magnetic field is fundamentally different from the real substance.

2.14 The Velocity of Motion of Magnetic Field may be Infinite.
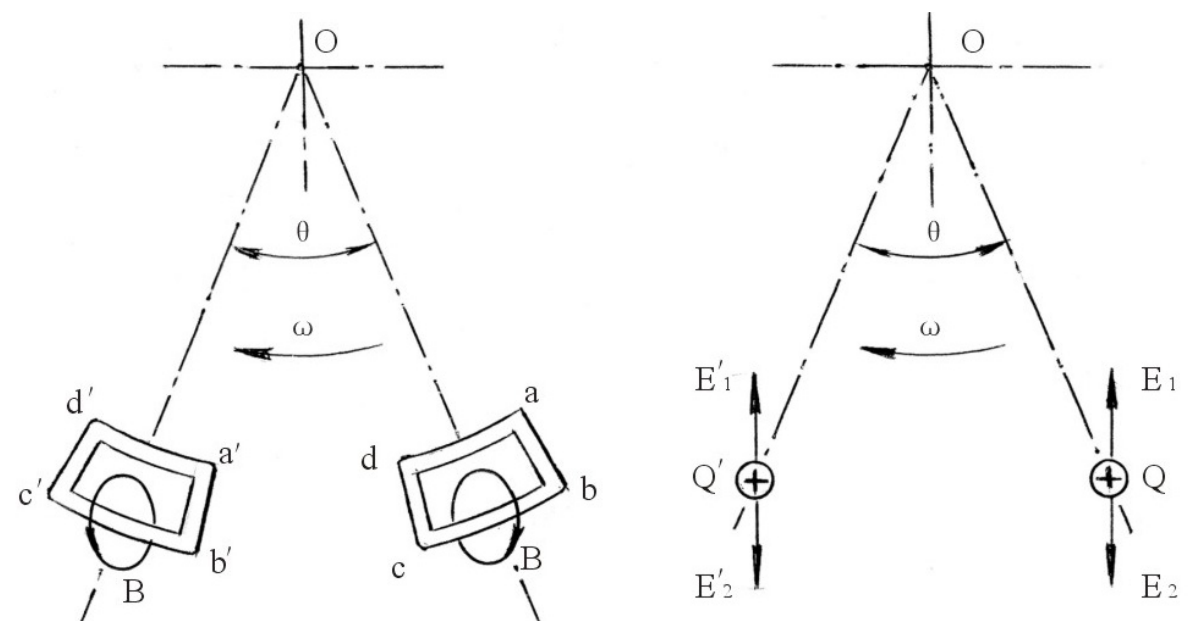

Figure 7. Fundamental difference between the moving magnetic field and the electric field

The magnetic field has been generated inside or outside the current-carrying coil abcd (as shown in Figure 7 (a)). The circular ring B represents a magnetic line of force of magnetic field. According to the principle of electromagnetics, the magnetic field generated by the coil $a b c d$ may extend to infinity. If the coil $a b c d$ is rotated around Point $\mathrm{O}$ at angular velocity $\omega$ in the clockwise direction, after $\theta / \omega$ seconds, the coil $a b c d$ will be rotated to the position $a^{\prime} b^{\prime} c^{\prime} d^{\prime}$ at the angle $\theta$. The magnetic line of force $\mathrm{B}$ will be also rotated to the position $a^{\prime} b^{\prime} c^{\prime} d^{\prime}$ together with $a b c d$. Obviously, in this case, the magnetic field of abcd is a rotating magnetic field (angular velocity $\omega$ ), in which the velocity of motion of magnetic field substance (distance $r$ between the magnetic field and the point O) shall be $V=\omega r$. If $r=C / \omega$ (c- the velocity of light), then $V=\omega c / \omega=c$. If $r>c / \omega$, then $V>C$. If $r \rightarrow \infty$, then $V \rightarrow \infty$. In other words, the velocity of motion of magnetic field may not only be greater than the velocity of light, but also can be infinite.

According to the principle of constancy of light velocity, it is noted that, the velocity of light is an extreme velocity in nature; the velocity of motion of any real substance can not be faster than the velocity of light. The example shows (Figure 7 (a)) that, the velocity of motion of magnetic field may not only be greater than the velocity of light, but also can be infinite, which shows with certainty that, the magnetic field can't be a kind of real substance.

Thus, it is natural to ask whether the velocity of motion of electric field around the charged body may also be greater than the velocity of light and even can be infinite when a charged body is being rotated according to the above reasoning. From the analysis, this possibility can not exist, because the electric field of electron or proton is an active field. When the electron or proton is rotated, the electric field around it can only move not be rotated (see Figure 7 (b)). When the electric charge $Q$ is rotated to the position $Q^{\prime}$ at angular velocity $\omega$, the electric line of force of $Q\left(E_{1} \& E_{2}\right)$ will become $E_{1}^{\prime}$ and $E_{2}^{\prime}$; in spite of the different position, the direction has not been changed. When the large electric charged body (consisting of a number of electrons) is rotated, the electric field of each electron or proton on the charged body can only move not be rotated, so that the entire electric field of charged body can only move not be rotated.

The above analysis shows that, some basic principles of electromagnetics can be considered as the summary of superficial phenomena (as it were), without further clarification regarding the root of such electromagnetic phenomena. It is noted that, there are some theoretical analysis errors of electromagnetics, e.g. both action and 
reaction may have the same direction, etc. It has not made a reasonable explanation of deflection results of charged particles in a magnetic field. It hasn't a definite answer about whether the magnetic field can be in motion. It has not made a clear and reasonable explanation for actual conditions of electromagnetic wave. Moreover, the analysis results (i.e. the velocity of motion of magnetic field can be greater than the velocity of light) have demonstrated that, the conclusion is wrong, that the magnetic field is a kind of real substance according to electromagnetics. The experimental results (existence of electrostatic field around the permanent magnet) show with certainty that, some of the basic principles of electromagnetics are not true universally. These facts are enough to prove that there are some defects in electromagnetics. Therefore, it is necessary to explore the inherent causes of all electromagnetic phenomena and add some new principles to eliminate the defects in electromagnetics.

\section{Basic Rule of Motion of Electric Field}

The previous part points out the basic defect of the is that it does not clarify the inherent reason and the physical process of the electromagnetic phenomena, therefore, in order to eliminate the defects in the electromagnetics, it is the first step to explore the reason of the electromagnetic phenomena and analyze the points in common of electromagnetic phenomena, and then identify the basic factors leading to all the electromagnetic phenomena. Since the basic factors are determined, the research filed can be also determined.

The analysis indicates that the one sharing point in all electromagnetic phenomena is that they are all accompanied with electronic motions of all forms inevitably; therefore, it can be known that the electronic motion is the source of all electromagnetic phenomena; and then through what should the electrons interact? The electron could affect another electron through the peripheral electric field obviously, which means the motion of the electric field shall be the basic factor of forming all electromagnetic phenomena; therefore, our direction of research is to study the law of motion of the electric field and analyze all forms of motion of all electric fields by using the laws determined.

The seven rules below can be summarized by analyzing the characteristics of the motion of the electric fields in all electromagnetic phenomena combining with some fundamentals in physics.

\subsection{Principle of Equivalent Electric Field}

According to the electromagnetics, the total intensity of the combined electric field at some point of two charges of identical or different polarities shall be the combination of the individual intensity of the electric field of each of two charges at this point, which is called superposition principle. The practices proved that the superposition principle should also be correct although the distance among the two charges is very small. This superposition principle indicates that the existence of the electric field around any charge should not be affected by the surrounding charges; therefore, it can be inferred that the negative electric field of the electron and the positive electric field of the proton should still exist in all materials, and the distribution condition of their electric fields should be the same as those of the electric field when all electrons and protons exist individually, that is to say, the electric field around the material should be equivalent to the one when all electrons and protons exist in the matter individually. This deduction shall be called equivalent electric field principle, from which it can be known that the electric fields around the free electrons in the matter shall exist forever with the free electrons, therefore, if the free electrons in the material are in macroscopic motion, the electric fields generated by the free electrons shall surely be in the same macroscopic motion (no kinetic electric field would be generated during the thermal motion of the electron in the matter and the rotation around the nucleus, see 3.6) with the free electrons. Thus, the electric field around the free electrons forming the electric current would implement the identical motion with the free electron inevitably when the electric current exists in the lead. This kinetic electric field should be called the magnetic field. Moreover, the magnetic induction intensity of any current element Idl ( $I$-electric current; $d l$-length of micro lead) on the lead at the place $r$ shall be as follows according to the Biot-Savart law:

$$
\left.d B=\frac{\mu_{0}}{4 \pi} \cdot \frac{d l}{r^{2}} I \times r_{0} \text { (Unit vector in direction of } r_{0}-\mathrm{r}\right)
$$

The linear charge density indicated by using $-\tau$ of the free electron on the lead shall be $I d l=-\tau(-V) d l=d Q V$ because the $I$ is formed by the free electrons in the lead actually during motion at speed of $-V$ (opposite to the direction of $I)$; and because $\mu_{0} \varepsilon_{0}=1 / C^{2}$ and $K=1 / 4 \pi \varepsilon_{0},\left(\mu_{0} / 4 \pi\right)=K / C^{2}$; therefore, $d \boldsymbol{B}=\frac{K}{C^{2}} \frac{d Q}{r^{2}} V \times r_{0}$ 
Because the electric field intensity of $d Q$ at the place $\mathrm{r}$ is $d \boldsymbol{E}=k \frac{d Q}{r^{2}} r_{0}$,

The above-mentioned formula can be transformed as $d \boldsymbol{B}=\frac{1}{C^{2}} V \times d E$,

or

$$
\boldsymbol{B}=\frac{1}{C^{2}} V \times E=\mu_{0} \varepsilon_{0} V \times E \ldots \ldots \ldots
$$

From the Formula (1), the magnetic field can be defined preliminarily: the magnetic field is the kinetic electric field. From this definition, it can be known that when there is the electric current in the lead or the moving electric charges in the space, the electric field generated by the free electrons or the moving electric charges will form the kinetic electric field, namely, the magnetic field.

\subsection{Contraction of Electric Field in Direction of Motion}

The special relativity pointed out that the length $l$ of the object in the direction $V$ would be contracted as $l^{\prime}=l \sqrt{1-\mathrm{V}^{2} / \mathrm{C}^{2}}$ when moving at the speed $V$. The length of the electric field in the direction $V$ would be contracted at the proportion $\sqrt{1-\mathrm{V}^{2} / \mathrm{C}^{2}}$ inevitably when moving at speed $V$ because the electric field is formed by substances.

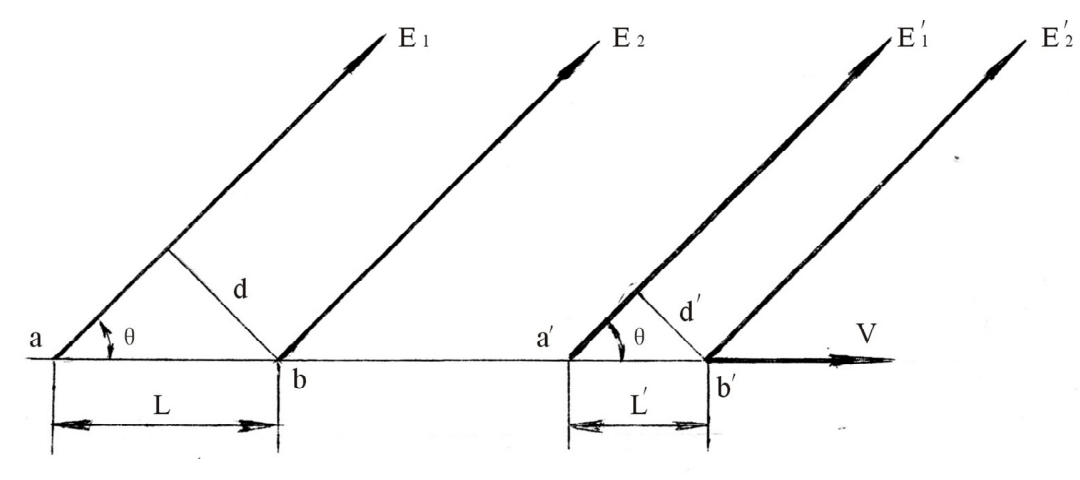

(a)

(b)

Figure 8. Increasing field intensity as a result of motion and contraction of the electric field

Figure 8 (a) indicates the static electric field; E1 and E2 are two power lines thereof, wherein the included angle $\theta$ is formed by the power lines and the axis $\mathrm{X} ; l$ indicates the distances from the intersection point a and the intersection point $\mathrm{b}$ of $\mathrm{E} 1$ and $\mathrm{E} 2$ and the axis $\mathrm{X}$; the distance between the power line $\mathrm{E} 1$ and the power line $\mathrm{E} 2$ is $d=l \sin \theta$ obviously. Figure 8 (b) indicates the $l^{\prime}=l \sqrt{1-\mathrm{V}^{2} / \mathrm{C}^{2}}$ can be formed after contraction of the (a) electric field moving to the direction $\mathrm{X}$ at speed $V$. The distance between the two power lines is contracted as $d^{\prime}=l^{\prime} \sin \theta=l \sin \theta \sqrt{1-V^{2} / C^{2}}=d \sqrt{1-V^{2} / C^{2}}$ which indicates that regardless of the moving direction of the electric field, the distance of the two power lines of the electric field before and after contraction: $d^{\prime}=d \sqrt{1-V^{2} / C^{2}}$.

Because the electric field intensity is in inverse proportion to the distance between the power lines thereof, thus

$$
E^{\prime}=E d / d^{\prime}=E \sqrt{1-V^{2} / C^{2}} \ldots \ldots
$$

From the Formula (2), the electric field intensity E shall be increased as $E / \sqrt{1-V^{2} / C^{2}}$ when the electric field moves at speed $V$. Moreover, we can further define the magnetic field as the electric field in motion and contraction.

The known electric field shall have the energy which may be increased inevitably when the motion intensity of the electric field is increased. The principle of equivalent electric field pointed out that the electric fields of the electrons and the protons around the substance shall still exist. Why could not their electric fields exist? Obviously it is because the electric field direction of the electron is opposite to that of the proton, which leads to the offset of the electric fields with each other. 
The electric field energy of the electron of one unit volume can be indicated by using $\omega_{+}=\varepsilon E^{2} / 2$ in the space without the motion electric field; and the electric field energy of the proton with identical power with the electron can be indicated by using $\omega_{-}=-\varepsilon E_{0}^{2} / 2$, in the formulas $\omega_{+}$and $\omega_{-}$, the absolute value of $E$ is equal to that of $E 0$. Moreover, when the electric field moving at speed $V$ is available in the space, the $\mathrm{E}$ shall be changed as $E^{\prime}=E / \sqrt{1-V^{2} / C^{2}}$ and the electric field energy of the electron can be changed as

$$
\omega_{+}=\frac{\varepsilon_{0}}{2}\left(\frac{E}{\sqrt{1-V^{2} / C^{2}}}\right)^{2}=\frac{\varepsilon_{0}}{2} \frac{E^{2}}{\left(1-V^{2} / C^{2}\right)}
$$

Because $1 /\left(1-V^{2} / C^{2}\right)$ can be expanded as $1+V^{2} / C^{2}+V^{4} / C^{4}+\ldots \ldots$

According to series, and the moving speed $\mathrm{V}$ of the common electric field is smaller than the speed of light, the $V^{4} / C^{4}$ and the items afterwards of the expansion equation can be negligible, and the above-mentioned formula can be:

$$
\omega_{+}^{\prime}=\frac{\varepsilon_{0}}{2} E^{2}\left(1+\frac{V^{2}}{C^{2}}\right)=\omega_{+}+\frac{\varepsilon_{0} E^{2} V^{2}}{2 C^{2}}
$$

The Formula (1) can be written as $B=\mu_{0} \varepsilon_{0} V E$, that is $V E=B / \mu_{0} \varepsilon_{0}$ when $V$ is vertical to $E$. Further because $\mu_{0} \varepsilon_{0}=1 / C^{2}$ and $B=\mu_{0} H$, the above-mentioned formula can be transformed as:

$$
\omega_{+}^{\prime}=\omega_{+} \frac{\varepsilon_{0}^{2} \mu_{0}\left(\mu_{0}^{2} H^{2}\right)}{2 \mu_{0}^{2} \varepsilon_{0}^{2}}=\omega_{+}+\frac{\mu_{0} H^{2}}{2}
$$

And the total energy of the unit volume in the space is:

$$
\omega=\omega_{+}+\omega_{-}=\omega_{+}+\frac{\mu_{0} H^{2}}{2}+\omega_{-}=\frac{\mu_{0} H^{2}}{2}
$$

Formula (3) indicates that the energy must be available when the motion electric field exists in the space, and the energy of the unit volume thereof shall be $\mu_{0} H^{2} / 2$; and this energy shall be the one to be increased during motion and contraction of the electric field. Moreover, this energy shall also be the so-called magnetic field energy in electromagnetics.

\subsection{Formula for Force Transformation}

The force of the stressed charged body in motion shall be moved jointly with the charged body. If it is known about the length, the mass and the time of the charged body in motion shall be transformed; would the force in motion thereof be transformed? The generally accepted common sense for force balancing should be explained before answering this question, and this is the force in balanced state will not be changed due to different observers. For example:

One object of $1 \mathrm{~kg}$ is placed on the spring scale, and the pointer of the spring scale is located at the scale of $1 \mathrm{~kg}$ not only for the people who is static with respective to spring scale but for any other people in motion. This fact indicates that the observers of any other reference systems could observe that one group of forces balanced in one reference system are in the balanced state. Or this group of forces balanced will not be unbalanced due to different reference systems which the observers belong to; this is the generally acceptance fact in daily lives of people, and I call this fact as the force-balancing immutable axiom. Moreover, we can deduce the formula of force transformation according to the force-balancing immutable axiom.

Figure 9a indicates the three forces $F_{A}, F_{B}$ and $F_{C}$ are in the balanced state, wherein the three forces can be the force applied to the electric field, or universal gravitation or spring force, etc. and in the diagram, the lengths of OA, OB and OC indicate $F_{A}, F_{B}$ and $F_{C}$ respectively; $F_{C}$ is parallel with the Line $\mathrm{X} ; F_{A}$ is equal to $F_{B}$, and the included angles $\theta$ (positive $\theta$ and negative $\theta$ are not taken into account) are formed with the Line X. $F_{A} \cos \theta+F_{B} \cos \theta=F_{C}$ because the three forces are balanced. Because $F_{A}$ is equal to $F_{B}$ and in order to have the simple deduction, we set $F_{A}=F_{B}=F$, thus $2 F \cos \theta=F_{C}$.

Figure $9 \mathrm{~b}$ indicates movements of the three forces parallel with the Line $\mathrm{X}$ in Figure $9 \mathrm{a}$ at speed $V$. The length of the direction $V$ (direction $\mathrm{X}$ ) shall be shortened according to special relativity; the $F_{c}$ of the Figure 9a shall be shortened as $F_{C}^{\prime}$; the $F_{A}$ and the $F_{B}$ shall be shortened as the $F_{A}^{\prime}$ and the $F_{B}^{\prime}$; the $F_{C}^{\prime}$, the $F_{A}^{\prime}$ and the $F_{B}^{\prime}$ are 
balanced according to the force-balancing immutable axiom; $F_{A}^{\prime}$ is equal to the $F_{B}^{\prime}$ certainly. Moreover, $2 F^{\prime} \cos \theta^{\prime}=F_{C}^{\prime}$ as well if $F^{\prime}=F_{A}^{\prime}=F_{B}^{\prime}$.

The relationship between $F_{c}$ and $F_{c}^{\prime}$ can be deduced according to the changes of the forces applied to the electric field during movement.

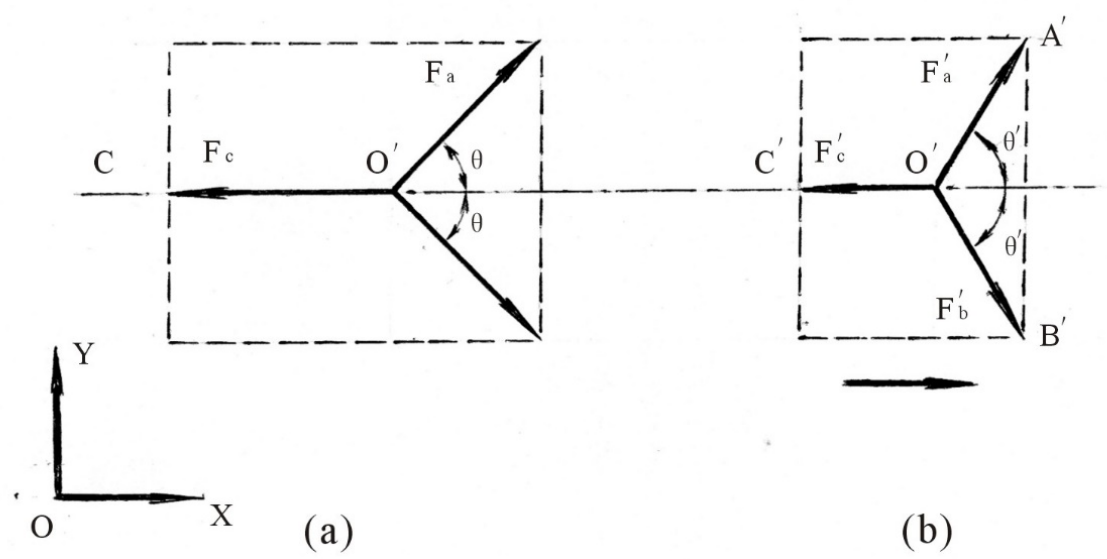

Figure 9. Changes of force during movement

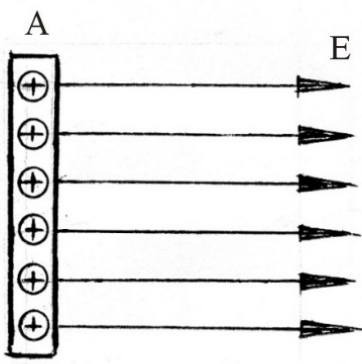

B

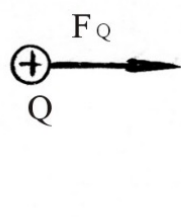

(a)

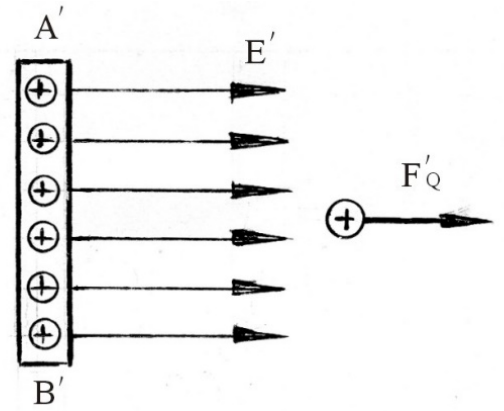

(b)

Figure 10. Electric field for movement of intensity line E of parallel electric field

Figure 10a indicates that the charge $Q$ is positioned in the infinite uniform electric field generated by the charged panel, wherein $\mathrm{E}$ indicates the electric field intensity; $A B$ indicates one part of cross section of the charged panel; and obviously the force applied to $Q$ is: $F_{Q}^{\prime}=E Q$. Figure $10 \mathrm{~b}$ indicates $A B$ becomes $A^{\prime} B^{\prime}$ after movement of $A B$ parallel with $E$ at speed $V$; and the length in the direction vertical to $V$ after movement will not be changed according to the special relativity, thus $A^{\prime} B^{\prime}=A B$, and the electric field intensity of $A^{\prime} B^{\prime}$ is: $E^{\prime}=E$. Therefore, the force applied to $Q$ in the $A^{\prime} B^{\prime}$ electric field is: $F_{Q}^{\prime}=E_{Q}^{\prime}=E Q=F_{Q}$. Seen from the fact, the force $F_{Q}$ will not be changed $\left(F_{Q}^{\prime}=F_{Q}\right)$ after movement in the direction parallel with $F_{Q}$. As for the force in Figure 10, it is inevitable that $F_{c}^{\prime}=F_{c}$. Moreover, it is inevitable that $F^{\prime} \cos \theta^{\prime}=F \cos \theta$ because $F_{c}^{\prime}=2 F^{\prime} \cos \theta^{\prime}$ and $F_{C}=2 F \cos \theta$.

$$
F^{\prime}=F \frac{\cos \theta}{\cos \theta^{\prime}}
$$

From the Figure 1

$$
\cos \theta=\frac{O D}{\sqrt{O D^{2}+A D}} \quad \cos \theta^{\prime}=\frac{O^{\prime} D^{\prime}}{\sqrt{O^{\prime} D^{\prime 2}+A^{\prime} D^{\prime}}}
$$


$A^{\prime} D^{\prime}=A D$ because the length in the direction vertical to the direction $\mathrm{V}$ will not be changed; while the length in the direction $\mathrm{V}$ is $O^{\prime} D^{\prime}$, and $O^{\prime} D^{\prime}=O D \sqrt{1-V^{2} / C^{2}}$ according to the transformation formula of the length of special relativity; therefore, $\cos \theta^{\prime}$ can be transformed as

$$
\begin{gathered}
\cos \theta^{\prime}=\frac{O D \sqrt{1-V^{2} / C^{2}}}{\sqrt{O D\left(1-V^{2} / C^{2}\right)+A D^{2}}} \\
F^{\prime}=F \frac{\cos \theta}{\cos \theta^{\prime}}=F \frac{O D}{\sqrt{O D^{2}+A D^{2}}} \cdot \frac{\sqrt{O D^{2}\left(1-V^{2} / C^{2}\right)+A D^{2}}}{O D \sqrt{1-V^{2} / C^{2}}} \\
=F \sqrt{\frac{\left(O D^{2}+A D^{2}\right)-O D^{2} V^{2} / C^{2}}{\left(O D^{2}+A D^{2}\right)\left(1-V^{2} / C^{2}\right)}}
\end{gathered}
$$

According to the equations of $A^{\prime} D^{\prime}$ and $O^{\prime} D^{\prime}$.

$$
\text { The } F^{\prime}=F \sqrt{\frac{1-\cos ^{2} \theta V^{2} / C^{2}}{1-V^{2} / C^{2}}}
$$

can be acquired by putting $\cos \theta^{\prime}=O D / \sqrt{O D^{2}+A D^{2}}$ to the above-mentioned formula.

The Formula (4) is the transformation formula of force; it is inevitable that $F=E Q$, that is, the force is in direct proportion to the electric field intensity as for the electric field force; therefore, $F^{\prime}=E^{\prime} Q$, that is, $E^{\prime}=E F^{\prime} / F$

$$
E^{\prime}=E \sqrt{\frac{1-\cos 2 \theta V^{2} / C^{2}}{1-V^{2} / C^{2}}}
$$

Then the relationship between $\cos \theta^{\prime}$ and $\cos \theta$ :

$$
\cos \theta^{\prime}=\cos \theta \sqrt{\frac{1-V^{2} / C^{2}}{1-\cos ^{2} \theta V^{2} C^{2}}}
$$

because $F^{\prime}=F \cos \theta / \cos \theta^{\prime}$ and $\cos \theta^{\prime}=F \cos \theta / F^{\prime}$

That the gravitational mass is not equal to the inertial mass could be proved easily by using the force-balancing immutable axiom because the sun and its planets are in the status in which the universal gravitation is balanced with the centrifugal force; therefore, one observer moving at high speed opposite to the sun could see this balanced status inevitably. If the gravitational mass is equal to the inertial mass, the universal gravitation of the sun to the planets would be increased according to $\left(1 / \sqrt{1-V^{2} / C^{2}}\right)^{2}$ while the centrifugal force of the planets to the sun would be increased according to $1 / \sqrt{1-V^{2} / C^{2}}$ when the observer moves opposite to the sun at the speed $\mathrm{V}$, and all planets may inevitably disappear completely by being adsorbed by the sun because the universal gravitation is greater than the centrifugal force, which is not in line with the force-balancing immutable axiom; so the gravitational mass cannot be equal to the inertial mass.

That the gravitational mass of the object has nothing to do with the velocity of movement of the object can also be proved easily by the force-balancing immutable axiom because one observer moving opposite to them at speed $\mathrm{V}$ can also see they are in the balanced state if two objects have identical negative charges, and the mutual universal gravitation among them is just equal to the mutual electrostatic repulsion. The two objects can be in the balanced state only when the gravitational mass has nothing to do with the velocity of movement of the object because the charge of the object is has nothing to do with the velocity of movement of the object. Moreover, it could be concluded that the gravitational mass, like the charge, is the constant which has nothing to do with the movement.

\subsection{Law of Relative Velocity}

Two positive charges Q1 and Q2 with velocities of movement of $V 1$ and $V 2$ are displayed in the In Figure 11 (a). Are the acting force and the reactive force between them are equal and opposite to each other? The magnetic induction intensity of Q2 generated at the place of Q1 is $B_{1}=\mu_{0} Q_{2} V_{2} \sin 90^{\circ} / 4 \pi r^{2}$ according to analysis on the basis of electromagnetic principle, and the Lorentz force of $Q_{1}$ is $F_{1}=\mu_{0} Q_{1} Q_{2} V_{1} V_{2} / 4 \pi r^{2}$ (the electrostatic 
repulsion between $Q 1$ and $Q 2$ is not discussed); and the magnetic induction intensity of Q1 generated at the place of $Q 2$ is $B_{2}=\mu_{0} Q_{1} V_{1} \sin 0 / 4 \pi r^{2}=0$, the Lorentz force of $Q 2: F_{2}=0$ which indicates that $F 1$ is not equal to $F 2$ obviously, and the directions thereof are not opposite to each other. Moreover, any movement condition of other $Q 1$ and $Q 2$ can be proved except the condition that $V 1$ is parallel with $V 2$; the interaction forces between the two are not equal and not opposite to each other, that is, both of them are not in line with the law of reaction. Moreover, we shall not discuss the general condition because the examples of Figure 11 (a) can illustrate the problem.

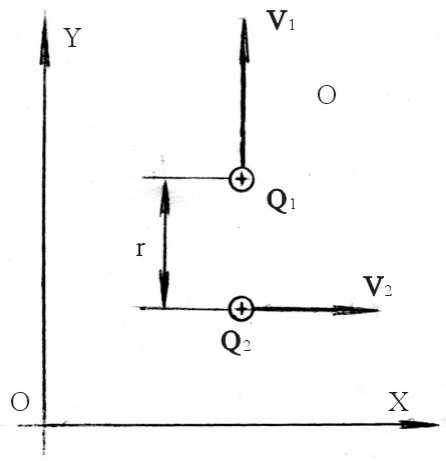

(a)

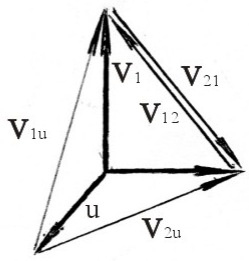

(b)

Figure 11. Analysis of relative velocity

The general results acquired by analyzing the Lorentz forces between two moving charges according to the principle of electromagnetics on the basis of the above-mentioned analysis were that the acting force and the reactive force were not equal and opposite to each other. Why did such an error analysis exist? From further analysis, the observers in different reference systems had the analytic results that were different from each other, which is not in line with the law of the reaction; for example, the other observer of the Figure 11 (b) moves opposite to us at speed $u$, and $V_{1}$ was changed as $V_{1 u}$, and $V_{2}$ was changed as $V_{2 u}$ in his view. Moreover, the analytic result of $B_{2 u} \neq 0$ analyzed by him was different from the above-mentioned result obviously, but was still not in line with the law of reaction.

The inevitable equal and opposite Lorentz forces between $Q 1$ and $Q 2$ should be taken as the balanced state in other form which is definitely not related to the reference system. And what of $Q 1$ and $Q 2$ was not related to the reference system? Seen from the analysis, the charges of $Q 1$ and $Q 2$ should be irrelevant to the reference system; and the relative velocity of the two should also be irrelevant to the reference system, see Figure 11 (b). Further, the velocity of movement of $\mathrm{Q} 1$ observed by other observer moving opposite to us at speed $u$ should be $\mathrm{V}_{\text {lu }}$, and the velocity of movement of $Q_{2}$ should be $V_{2 u}$, wherein the synthetic velocity of $V_{1 u}$ and $V_{2 u}$ should be $V_{12}$ (or $V_{21}$ ) and should be the synthetic velocity of $\mathrm{V}_{1}$ and $\mathrm{V}_{2}$; therefore, the relative velocity $\mathrm{V}_{12}$ or $\mathrm{V}_{21}$ of between Q1 and Q2 should be the objective velocity which is irrelevant to the reference system to which the observer belongs. To conclude on the basis of above-mentioned analysis, the relative velocity of the two charged objects should be taken as the sole standard velocity in the analysis of the interaction force of any two charged objects, wherein this rule should be called the law of relative velocity. Moreover, $\sin \theta$ of B1 and B2 in Figure 11 (a) should be the same inevitably ( $\theta$ indicates the included angle formed by the field intensity and the synthetic velocity) according to the law of relative velocity on the basis of analysis; therefore, it should be inevitable that $F_{1}=-F_{2}$.

\subsection{Transference Theorem of Force}

In Figure 12, the constant force $F$ is exerted on the sliding block $A$ which pushes the sliding block $A$ to move with distance $S$ within time $t$ when moving on the surface of the object $m$ at speed $V$, wherein the work done by the force $F$ is: $W_{1}=F S$. The practices prove that the kinetic energy acquired by the object $m$ is: $W_{2}=m(F t / m)^{2} / 2=F^{2} t^{2} / 2 m$; and $S=F t^{2} / 2 m$ because $W_{1}=W_{2}$; the movement distance of $m$ indicates that the force $F$ exerted to $m$ is identical to the static force of the sliding block. 


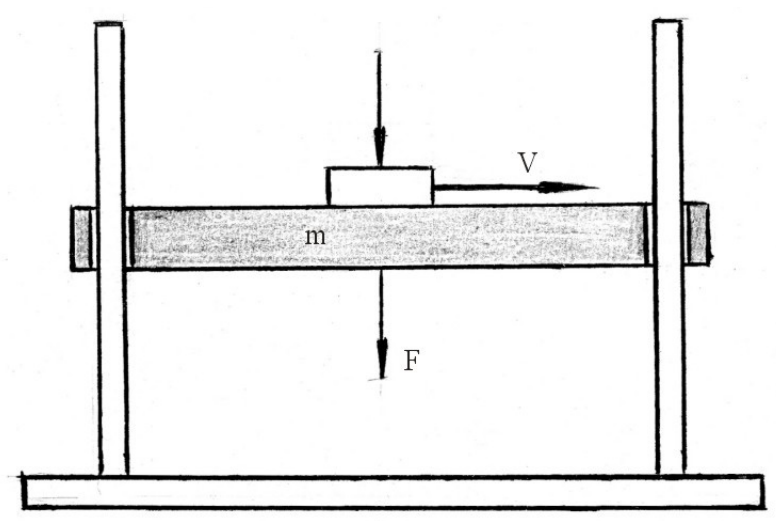

Figure 12. The force will not be changed after transferring of the force

3.3 Formula for Force Transformation indicates that the force $F$ moving at speed $V$ should be transformed as $F^{\prime}=F / \sqrt{1-V^{2} / C^{2}}(F \perp V)$, that is, the force we saw exerted on the sliding block should be the force $F^{\prime}$ in motion, wherein $F^{\prime}$ should be static $F$ compared to the sliding block rather than be $F^{\prime}$ when shifted on the static $\mathrm{m}$. And it indicated that when the force that was static with respect to A would not be changed when transferred as the force which was static with respect to B. Moreover, the power which was static with respect to the object should be taken as the original force, and that the original force shall not be changed when transferring is called the Original Force Transference Theorem.

\subsection{The States of Motion of the two Electric Fields having Identical Direction and Opposite Directions of Motion would be Offset}

The above-mentioned 3.1 indicated that the electric fields around the electrons and the protons in all substances still exist; and 3.2 indicated that the lengths thereof in the movement direction should be subjected to contraction inevitably during movement of the electric field, and the field intensities thereof should be increased inevitably. The velocity of the electrons in all substances during the movement of the electrons around the nucleus should be very great, and the thermal motions of the electrons in the objects should have certain velocity now that such an inexorable law was available, and why were the electric fields around these electrons in motion not subjected to contraction and increased? Why does the negative electrostatic field not appear around all substances? How to explain this fact?

The practices has proved that the electric field is the real substance and the length contraction of the substance in the movement direction is the correct inference of the special relativity. That the negative electrostatic field did not exist around the substance should also be affirmed when the two conclusions were affirmed, that is, the electric field of the electron in motion did not exist, and that the rotation and thermal motion of the electron around the nucleus should be affirmed; then the negative electric field in motion did not exist around the substance only possibly when the motion state of the two electric fields with the same direction of field and the opposite movement direction is offset with each other. If this becomes true, because the probability of all the electrons in the substance moving in all directions is the same and if there is electrons moving in some direction, there is bound to be electrons moving in opposite direction, their moving electric fields are inevitably be offset with each other to become the motionless electric fields. Therefore, the motion electric fields of all electrons should be offset inevitably, and the total effect should be inevitably that the electric fields in motion would not exist around the substance.

On the basis of the analysis above, as for the interaction effect of the moving electric fields, we shall conclude that the moving state of two electric fields with the same direction and the opposite movement direction shall be offset with each other. Seen from analysis of one section of current-carrying conductor folded together according to the above deduction, the two leads folded had opposite current directions, and the directions of motion of the electric fields of the free electrons thereof were opposite although each of the leads had the electric field moving along with the free electrons; moreover, the states of motion of the electric fields positioned in the identical direction and moving in the opposite directions should be offset inevitably and changed as the motionless electric fields. 
3.7 The Variable Velocity Motion of the Electron shall be Propagated in the Electric Field where the Electron Generates at the Light Speed during variable Motion of the Electron.

The propagation of the variable velocity motion of the electron in the electric field could be explained vividly by using the variation of the power line. Supposing that the electron implements the simple harmonic oscillation in the direction $\mathrm{Y}$, every point of all power lines of the electron shall implement the identical simple harmonic oscillation sequentially taking the electron as the beginning; and the sequential propagation velocity shall refer to the light speed. For example, one sine (or cosine) curve starting from the electron shall be formed by all points of the power line in the direction $\mathrm{X}$ doing the simple harmonic oscillation one after another from the electron. Moreover, this curve is similar to the ripple on the water whose points shall move vertically but not horizontally.

\section{Isokinetic motion of electric field}

\subsection{Isokinetic Motion of Point Charge}

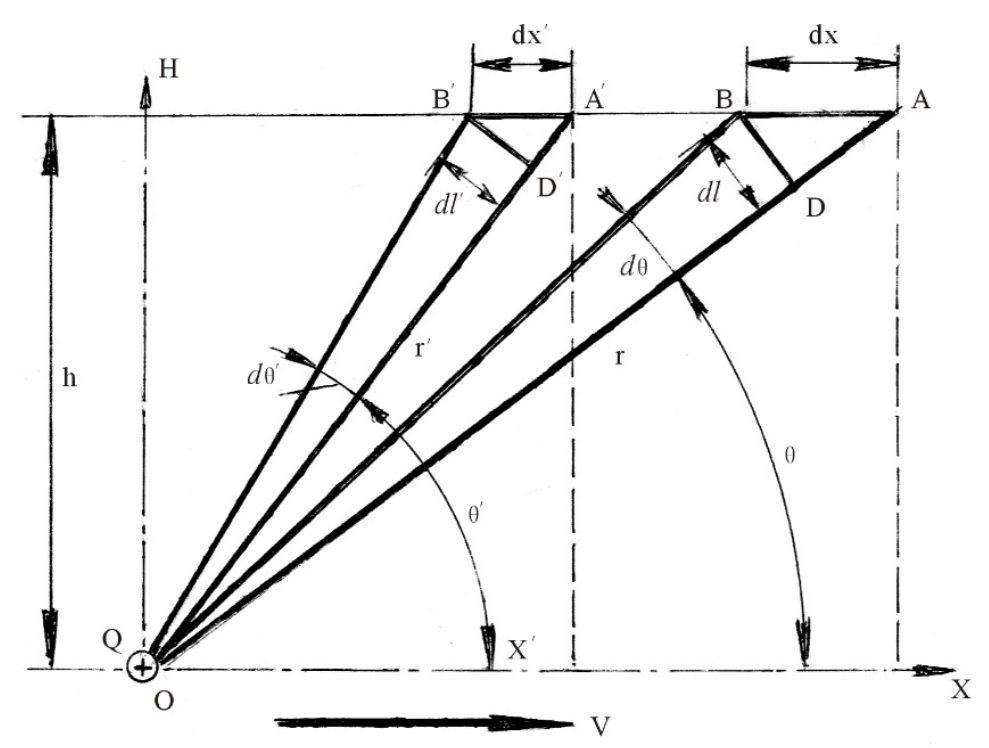

Figure 13. Contraction relationship of electric field when the point charges move

The point charge $\mathrm{Q}$ moves along the direction $\mathrm{OX}$ at the speed $\mathrm{V}$, as shown in Figure 13; and $O X A H$ indicates that the charge passes through the section of one regional electric field of the charge $\mathrm{Q}$ when not moving. The charge moves at the speed $\mathrm{V}$ now and the electric field contracts in the direction of motion in accordance with 3.2, wherein ox shall be contracted to ox' ( $\mathrm{x}$ indicates ox, $\mathrm{x}^{\prime}$ indicates ox' below); $\mathrm{dx}$ shall be contracted to $\mathrm{dx}$; ; therefore, the relationship of length transform according to the special theory of relativity can be expressed as $x^{\prime}=x \sqrt{1-V^{2} / C^{2}}$, $d x^{\prime}=d x \sqrt{1-V^{2} / C^{2}}$. Moreover, the section OXAH of the regional electric field shall be contracted as $\mathrm{O}^{\prime} \mathrm{X}^{\prime} \mathrm{A}^{\prime} \mathrm{H}^{\prime}$. In the figure, $d l$ indicates $B D, d l^{\prime}$ indicates $B^{\prime} D^{\prime} ; \quad$ in $\triangle B O D$ and $\triangle B^{\prime} O^{\prime} D^{\prime}, \quad O A=O B=r$ and $O A^{\prime}=O B^{\prime}=r^{\prime}$ because $d x, d x^{\prime}, d \theta$ and $d \theta^{\prime}$ are dimensionless; therefore $d l=r \cdot d \theta$ and $d l^{\prime}=r^{\prime} \cdot d \theta^{\prime}$

$$
\frac{d \theta}{d \theta^{\prime}}=\frac{d l \cdot r^{\prime}}{d l^{\prime} \cdot r}
$$

From the right angle $\triangle A B D$ and right angle $\triangle A^{\prime} B^{\prime} D^{\prime}$

$$
\begin{gathered}
d l=d x \cdot \sin \theta=d x^{\prime} \cdot \sin \theta / \sqrt{1-V^{2} / C^{2}} \\
d l^{\prime}=d x^{\prime} \cdot \sin \theta^{\prime}
\end{gathered}
$$

The relation $\frac{d \theta}{d \theta^{\prime}}=\frac{d x^{\prime} \cdot \sin \theta / \sqrt{1-V^{2} / C^{2}} \cdot r^{\prime}}{d x^{\prime} \cdot \sin \theta^{\prime} \cdot r}=\frac{\sin \theta \cdot r^{\prime}}{\sin \theta^{\prime} \cdot r \sqrt{1-V^{2} / C^{2}}}$ can be acquired when $d l$ and $d l^{\prime}$ are substituted into $d \theta / d \theta^{\prime}$.

From the figure, 


$$
\begin{gathered}
\sin \theta=\frac{h}{\sqrt{x^{2}+h^{2}}}=\frac{h}{\sqrt{h^{2}+x^{\prime 2} /\left(1-V^{2} / C^{2}\right)}}=h \sqrt{\frac{1-V^{2} / C^{2}}{x^{\prime 2}+h^{2}-V^{2} h^{2} / C^{2}}} \\
\sin \theta^{\prime}=h / \sqrt{x^{\prime 2}+h^{2}} \\
r=\sqrt{x^{2}+h^{2}}=\sqrt{\frac{x^{\prime 2}+h^{2}-V^{2} h^{2} / C^{2}}{1-V^{2} / C^{2}}} \\
r^{\prime}=\sqrt{x^{\prime 2}+h^{2}}
\end{gathered}
$$

The equation $\frac{d \theta}{d \theta^{\prime}}=\frac{\sqrt{1-V^{2} / C^{2}}}{1-V^{2} \sin ^{2} \theta^{\prime} / C^{2}}$ can be acquired when $\sin \theta, \sin \theta^{\prime}, r$ and $r^{\prime}$ are substituted into $d \theta / d \theta^{\prime}$.

The solid angle of $d \theta$ after surrounding one cycle of $O X$ shall be:

$$
d \Omega=d \theta \cdot \frac{2 \pi h}{r}=\frac{2 \pi h d \theta}{\sqrt{x^{2}+h^{2}}}=\frac{2 \pi h \sqrt{1-V^{2} / C^{2}}}{\sqrt{x^{\prime 2}+h^{2}-V^{2} h^{2} / C^{2}}} \cdot d \theta
$$

The solid angle of after surrounding one cycle of $O X$ shall be:

$$
d \Omega^{\prime}=d \theta^{\prime} \cdot \frac{2 \pi h}{r^{\prime}}=\frac{2 \pi h}{\sqrt{x^{\prime 2}+h^{2}}} \cdot d \theta^{\prime}
$$

The area of $\mathrm{E}$ at the place $\mathrm{r}$ shall be $r^{2} d \Omega$, and the area of $\mathrm{E}^{\prime}$ at $\mathrm{r}^{\prime}$ shall be $r^{\prime 2} d \Omega^{\prime}$ because the electric flux of $d \Omega$ is identical with that of $d \Omega^{\prime}$ and it is known that the field strength at the place $\mathrm{r}$ in $d \Omega$ is $\mathrm{E}, d \Omega$ is contracted as $d \Omega^{\prime}, \mathrm{r}$ is changed as $\mathrm{r}^{\prime} ; E^{\prime}$ indicates the change of the field strength. Moreover, the field strength is inversely proportional to the area on the promise that the electric flux is not changed; therefore

$$
\frac{E^{\prime}}{E}=\frac{d \Omega r^{2}}{d \Omega^{\prime} r^{\prime}}
$$

The formula below can be acquired when $d \Omega, d \Omega^{\prime}$ and $\sin \theta^{\prime}=h / \sqrt{x^{\prime 2}+h^{2}}$ are substituted into the last formula:

$$
\begin{gathered}
\frac{E^{\prime}}{E}=\frac{\sqrt{1-V^{2} / C^{2}}}{\sqrt{1-V^{2} / C^{2}\left[h^{2} /\left(x^{\prime 2}+h^{2}\right)\right]} \cdot \frac{d \theta}{d \theta^{\prime}} \cdot \frac{r^{2}}{r^{\prime 2}}} \\
=\frac{r^{2}}{r^{\prime 2}} \cdot \sqrt{\frac{1-V^{2} / C^{2}}{1-V^{2} \sin ^{2} \theta^{\prime} / C^{2}}} \cdot \frac{\sqrt{1-V^{2} / C^{2}}}{1-V^{2} \sin ^{2} \theta^{\prime} / C^{2}} \cdot \frac{r^{2}}{r^{\prime 2}} \\
E^{\prime}=\frac{1-V^{2} / C^{2}}{\left(1-V^{2} \sin ^{2} \theta^{\prime} / C^{2}\right)^{3 / 2}} \cdot \frac{r^{2}}{r^{\prime 2}} \cdot E
\end{gathered}
$$

Because $E=k Q / r^{2}$,

$$
E^{\prime}=K \frac{1-V^{2} / C^{2}}{\left(1-V^{2} \sin ^{2} \theta^{\prime} / C^{2}\right)^{3 / 2}} \cdot \frac{Q}{r^{\prime 2}}
$$

The Formula (7) is the distribution formula of electric field strength of the moving electron. Obviously, this formula is identical with the deduced result of electrodynamics.

The previous 3.3 indicates that the relation between the field strength $E^{\prime}$ after contraction when the charge moves and the field strength $E$ when the charge is static shall be expressed as using the Formula (5) 
$E^{\prime}=E \sqrt{1-\cos ^{2} \theta V^{2} / C^{2}} / \sqrt{1-V^{2} / C^{2}}$ which is different from the Formula (6) greatly in terms of form. Are the calculations of the two formulas the same? The analysis showed that the Formula (5) and the Formula (6) are the same formula. The Formula (7) can be acquired after simplification if the formula $\quad r^{\prime}=\sqrt{x^{\prime 2}+h^{2}}=\sqrt{r^{2} \cos ^{2} \theta\left(1-V^{2} / C^{2}\right)+r^{2} \sin ^{2} \theta}=r \sqrt{1-\cos ^{2} \theta V^{2} / C^{2}}$ and $\sin \theta^{\prime}=\sin \theta r / r^{\prime} \quad$ are substituted into the Formula (5) and the member and the denominator of the Formula (5) are multiplied by $\left(1-\cos ^{2} \theta V^{2} / C^{2}\right)\left(1-V^{2} / C^{2}\right)$. Moreover, the scopes of application of the Formula (5) and the Formula (7) are different; and the Formula (5) shall be applied to calculation of the $E^{\prime}$ contracted after moving of the charge if the included angle $\theta$ of $E$ and $V$ is known only when the charge is static; and the Formula (7) shall be taken to acquire the electric field distribution formula of the moving charge.

The Formula (7) has the another characteristics, that is, $E^{\prime}<E$ if $\theta<54.7^{\circ}$ and $E^{\prime}>E$ if $\theta>54.7^{\circ}$ on the premise that the moving speed of the charge is not great and $V<0.1 C$, namely $54.7^{\circ}$ is the field strength after moving of the charge and shall be taken as the critical angle of increasing and reduction.

4.1.1 Interaction Force among two Moving Charges

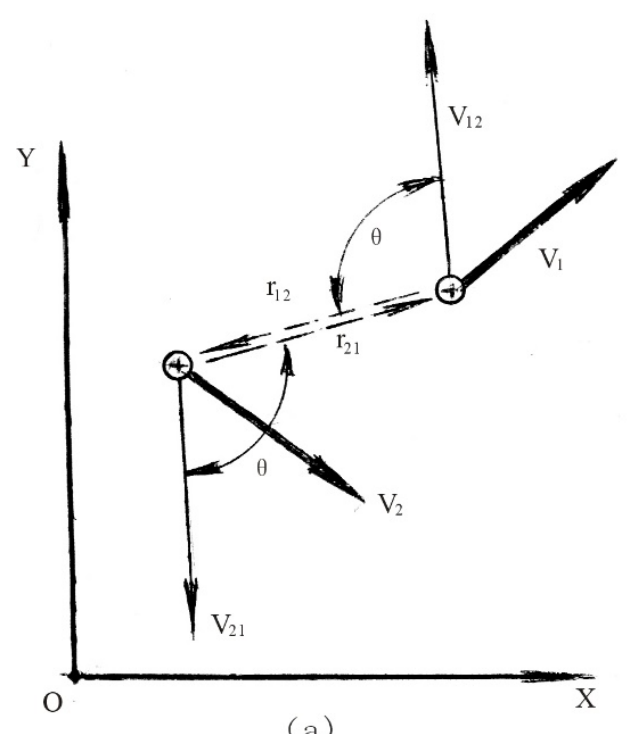

(a)

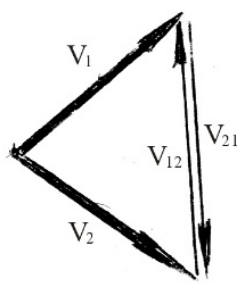

(b)

Figure 14. Interaction force of two moving charges

Two positive charges $Q 1$ and $Q 2$ moves at speed $V 1$ and $V 2$ respectively, and the $V 1$ and $V 2$ are located in the plane XOY, as shown in Figure 14 (a); the relative speed of the two charged bodies shall be taken as the exclusive analysis speed when analyzing the interaction force among the two moving bodies charged; while the total speed chart of $V_{1}$ and $V_{2}$ is shown in the Figure 14 (b). The relative speeds of $V_{12}$ and $V_{21}$ of the two bodies are equal and opposite in direction obviously. For $Q 2$, the electric field generated by $Q 1$ to $Q 2$ shall be contracted in accordance with the proportion of $\sqrt{1-V^{2} / C^{2}}$ in the direction $V_{12} ; \theta$ indicates the included angle formed by the $V_{12}$ and the distances $\mathrm{r}_{12}$ (which are also the direction of the electric field $Q 1$ at the place of $Q 2$ ) among $Q 1$ and $Q 2$. Moreover, the field strength of $Q 1$ at the place $Q 2$ shall be as follows according to the Formula (7) in 4.1:

$$
\boldsymbol{E}_{12}=K \frac{1-V_{12}{ }^{2} / C^{2}}{\left(1-V_{12}^{2} \sin ^{2} \theta^{\prime} / C^{2}\right)^{3 / 2}} \cdot \frac{Q}{r_{12}^{2}} \cdot \boldsymbol{r}_{12}{ }^{\circ} \quad \text { (The unit vector in the direction } \boldsymbol{r}_{12}^{\circ}-r_{12} \text { ) }
$$

Similarly, the field strength of $Q 2$ at the place $Q 1$ shall be

$$
\boldsymbol{E}_{21}=K \frac{1-V_{21}{ }^{2} / C^{2}}{\left(1-V_{21}^{2} \sin ^{2} \theta^{\prime} / C^{2}\right)^{3 / 2}} \cdot \frac{Q}{r_{21}{ }^{2}} \cdot \boldsymbol{r}_{21} \text { (The unit vector in the direction } \boldsymbol{r}_{21}^{\circ}-r_{21} \text { ) }
$$

It can be seen that the field stress $\boldsymbol{F}_{2}=\boldsymbol{E}_{12} Q_{2}$ exerted to $Q 1$ and the field stress $\boldsymbol{F}_{1}=\boldsymbol{E}_{21} Q_{1}$ exerted to $Q 2$ are equal and opposite in direction, that is, $\boldsymbol{F}_{2}=-\boldsymbol{F}_{1}$ 
On the basis of the above-mentioned analysis and conclusion, the previous 2.6 can be explained easily, specifically the interaction force of the moving charge and the current-carrying coil. The acting forces and the reacting forces among the moving charge and all free electrons of the current-carrying coil and among corresponding protons shall be equal and opposite in direction according to the conclusion that the acting forces and the reacting forces among two charged bodies are inevitably equal and opposite in direction. Therefore, the overall effect shall be inevitably in line with the law of reaction.

As for the Figure 3, whether A and B rotate when the trolley moves can be explained more easily. Moreover, the electrostatic force exists among A and B only, and no Lorentz force exists because A and B are relatively static; therefore, no rotation will occur naturally.

\subsection{Isokinetic motion of line electron beam}

The electric field around the free electrons in the wire with current still exists and moved along with the free electrons according to the theory of equivalent electric field in the previous 3.1 ; therefore, the free electrons in the long and straight wire with current are the most common movement of electron beam. If $\tau$ indicates the line electron density of the straight charged body within the unit length according to the electrostatics, the electric field strength at the place $r$ outside the charged body shall be:

$$
\left.\boldsymbol{E}=K \frac{2 \tau}{r} \boldsymbol{r}_{0} \text { (The unit vector in the direction } \boldsymbol{r}_{0}-r\right)
$$

The field strength after contraction shall exist inevitably when the charged body moves along the direction of the axis thereof at the speed $V$ according to the condition that the last electric field shall be contracted in the direction of motion at the proportion of $\sqrt{1-V^{2} / C^{2}}$ :

$$
\boldsymbol{E}^{\prime}=\boldsymbol{E} / \sqrt{1-V^{2} / C^{2}}=K 2 \tau \boldsymbol{r}_{0} / r \sqrt{1-V^{2} / C^{2}}
$$

4.2.1 Interaction force of two parallel wires with currents

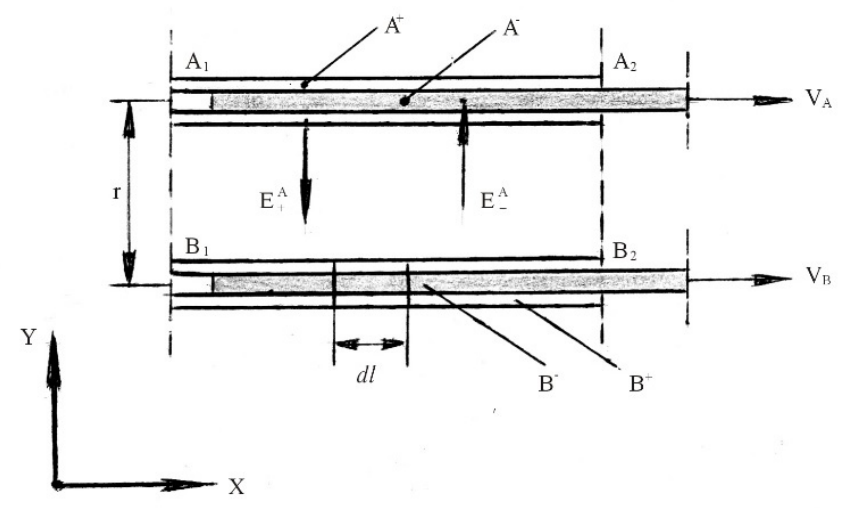

Figure 15. Interaction force of parallel wires with currents

$A_{1} A_{2}$ and $B_{1} B_{2}$ indicate one section of two parallel infinite straight leads respectively, as shown in Figure 15. The wire with current is described by using the pipeline and the flux in order to clarify clearly, wherein the flux indicates the free electron beam (current) of macro motion in the wire with current; and the pipeline indicates the proton having the electricity equivalent to that of the free electrons.

In order to facilitate analysis, suppose that the velocities of movement of the free electrons of the pipeline $\mathrm{A}$ are $V_{A}$, the velocities of movement of the free electrons of the pipeline $\mathrm{B}$ are $V_{B}$, the linear charge density of the free electrons of the pipeline A are $\tau_{A}^{-}$and the linear charge density of the free electrons of the proton pipeline are $\tau_{A}^{+}$, $\tau_{A}^{-}=-\tau_{A}^{+}$obviously; and as for the pipeline $\mathrm{B}, \tau_{B}^{-}=-\tau_{B}^{+}$similarly.

The positive electricity $d Q_{+}=\tau_{B}^{+} d l$ and the negative electricity $d Q_{-}=\tau_{B}^{-} d l$ in $d l$ after analyzing the acting force of one section of micro lead $d l$ on the lead A on the lead B which has the distance far from the lead A. It can be seen from $d Q^{+}$in $d l$, the field strength of the electrostatic field generated by $A^{+}$shall be $\boldsymbol{E}_{Q_{+}^{+}}^{A_{+}}=K 2 \tau_{A}^{+}\left(-\boldsymbol{Y}_{0}\right) / r$ (the 
unit vector in the direction $\boldsymbol{Y}_{0}-r$ ); and seen from $d Q_{+}$, one electric field moving at the speed $V_{A}$ is generated by the free electron $A^{-}$, and the field strength should be $\left(\tau_{A}^{-}=-\tau_{A}^{+}\right)$according to the Formula (8)

$$
\boldsymbol{E}_{Q^{+}}^{A^{-}}=K 2 \frac{\left(-\tau_{A}^{+}\right)}{r} \cdot \frac{-\boldsymbol{Y}_{0}}{\sqrt{1-V^{2} / C^{2}}}=K \frac{2 \tau_{A}^{+}}{r} \cdot \frac{\boldsymbol{Y}_{0}}{\sqrt{1-V_{A}^{2} / C^{2}}}
$$

Therefore, the total field strength felt by $d Q_{+}$should be

$$
\boldsymbol{E}_{Q+}=\boldsymbol{E}_{\varrho+}^{A+}+\boldsymbol{E}_{\varrho+}^{A-}=2 K \tau_{A}^{+}\left(\frac{1}{\sqrt{1-V_{A}^{2} / C^{2}}}-1\right) \frac{\boldsymbol{Y}_{0}}{r}
$$

$d Q_{+}$shall be attracted by the lead A if $\boldsymbol{E}_{Q_{+}}$was positioned in the direction $\boldsymbol{Y}_{0}$ obviously. $V_{A}^{4} / C^{4} . V_{A}^{6} / C^{6} \ldots$ can be ignored because $d Q_{+}$can be expanded according to the above-mentioned series as $1+V_{A}^{2} / 2 C^{2}+3 V_{A}^{4} / 8 C^{4}+5 V_{A}^{6} / 16 C^{6}+\ldots, V_{A} / C$ which is minimal because of $1 / \sqrt{1-V_{A}^{2} / C^{2}}$; therefore, the last formula could be transformed into

$$
\boldsymbol{E}_{Q^{+}}=2 K \tau_{A}^{+}\left(1+V_{A}^{2} / 2 C^{2}-1\right) \boldsymbol{Y}_{0} / r=K \tau_{A}^{+} V_{A}^{2} \boldsymbol{Y}_{0} / C^{2} r
$$

And the force exerted to $d Q_{+}$shall be

$$
d \boldsymbol{F}_{Q^{+}}=\boldsymbol{E} d Q_{+}=\boldsymbol{E} \tau_{B}^{+} d l=K \tau_{A}^{+} \tau_{B}^{+} d l V_{A}^{2} \boldsymbol{Y}_{0} / C^{2} r
$$

Then seen from the internal part of $d l$, the field stress $d F_{Q_{-}}$from A is exerted to the free electron $d Q_{-}$; and as for $d Q_{-}, A^{+}$moves at the speed $V_{B}$; therefore, the field strength generated by $A^{+}$to $d Q_{-}$shall be

$$
\boldsymbol{E}_{Q^{-}}^{A+}=2 K \tau_{A}^{+}\left(-\boldsymbol{Y}_{0}\right) / r \sqrt{1-V_{B}^{2} / C^{2}}
$$

The formula can be simplified as $\boldsymbol{E}_{Q-}^{A+}=2 K \tau_{A}^{+}\left(-\boldsymbol{Y}_{0}\right)\left(1+V_{B}^{2} / 2 C^{2}\right) / r$ after being expanded according to the above-mentioned series.

Then seen from the field generated by $A^{-}$to $d Q_{-}$, the interaction of $A^{-}$and $d Q_{-}$must be analyzed in accordance with the relative speeds thereof on the basis of explanation in previous 3.4, and the velocity of movement of $A^{-}$opposite to $d Q_{-}$is $V_{A}-V_{B}$; therefore, the field strength generated by $A^{-}$opposite to $d Q_{-}$ shall be

$$
\begin{aligned}
\boldsymbol{E}_{Q^{--}}^{A-} & =2 K\left(-\tau_{A}^{+}\right)\left(-\boldsymbol{Y}_{0}\right) / r \sqrt{1-\left(V_{A}-V_{B}\right)^{2} / C^{2}} \\
& =2 K \tau_{A}^{+} \boldsymbol{Y}_{0}\left[1+\left(V_{A}^{2}-V_{B}^{2}\right) / 2 C^{2}\right] / r
\end{aligned}
$$

The total field strength of A exerted to $d Q_{\text {. }}$ shall be

$$
\begin{aligned}
\boldsymbol{E}_{Q-} & =\boldsymbol{E}_{Q-}^{A+}+\boldsymbol{E}_{Q-}^{A-}=2 K \tau_{A}^{+} \boldsymbol{Y}_{0}\left(V_{A}^{2}-2 V_{A} V_{B}+V_{B}^{2}-V_{B}^{2}\right) / 2 C^{2} r \\
& =K \tau_{A}^{+} \boldsymbol{Y}_{0}\left(V_{A}^{2}-2 V_{A} V_{B}\right) / C^{2} r
\end{aligned}
$$

The acting force exerted to $d Q_{-}$shall be

$$
\begin{aligned}
d \boldsymbol{F}_{Q-} & =\boldsymbol{E}_{Q-} d Q_{-}=K \tau_{A}^{+}\left(-\tau_{B}^{+}\right) d l \boldsymbol{Y}_{0}\left(V_{A}^{2}-2 V_{A} V_{B}\right) / C^{2} r \\
& =-K \tau_{A}^{+} \tau_{B}^{+} d l \boldsymbol{Y}_{0}\left(V_{A}^{2}-2 V_{A} V_{B}\right) / C^{2} r
\end{aligned}
$$

What should be explained is that the acting force $d \boldsymbol{F}_{Q_{-}}$acting on $d Q_{-}$moves along $d Q_{-}$at the speed $V_{B}$, and the force of motion is required to transform; therefore, $d \boldsymbol{F}_{Q_{-}}$shall be transformed as $d F_{Q_{-}}^{\prime}$. However, the force to be transformed shall act on the immobile lead (on the pipeline wall as shown in the figure) and was 
required to transform into the former force $d F_{Q_{-}}$according to the transference theorem of former force in 3.5.

The total force exerted to $d Q_{+}$and $d Q_{-}$in $d l$ from A shall be

$$
\begin{aligned}
d \boldsymbol{F} & =d F_{Q^{+}}+d F_{Q-}=K \tau_{A}^{+} \tau_{B}^{+} d l \boldsymbol{Y}_{0}\left[V_{A}^{2}-\left(V_{A}^{2}-2 V_{A} V_{B}\right)\right] / C^{2} r \\
& =2 K \tau_{A}^{+} \tau_{B}^{+} V_{A} V_{B} d l \boldsymbol{Y}_{0} / C^{2} r
\end{aligned}
$$

Because $\tau_{A}^{+} V_{A}=I_{A}, \tau_{B}^{+} V_{B}=I_{B}$ and $K=1 / 4 \pi \varepsilon_{0}, \varepsilon_{0} \mu_{0}=1 / C^{2}\left(K / C^{2}\right)=\mu_{0} / 4 \pi$, the last formula can be transformed into

$$
d \boldsymbol{F}=\frac{\mu_{0}}{4 \pi} \cdot \frac{2 I_{A} I_{B} d l}{r} \boldsymbol{Y}_{0}
$$

And the force $\mathrm{Fl}$ exerted to the lead with length of 1 long of the Bwire with current shall be

$$
\boldsymbol{F}_{l}=\int_{0}^{l} d \boldsymbol{F}=\frac{\mu_{0}}{4 \pi} \cdot \frac{2 I_{A} I_{B} l}{r} \boldsymbol{Y}_{0}
$$

The Formula (9) indicates that $\boldsymbol{F}_{l}$ is located in the direction $\boldsymbol{Y}_{0}$, that is, the lead B is attracted and guided by A if the directions of the currents $I_{A}$ and $I_{B}$ are the same; $\boldsymbol{F}_{l}$ is located in the direction $-\boldsymbol{Y}_{0}$ and the lead B shall be repelled by $\mathrm{A}$ if the directions of the currents $I_{A}$ and $I_{B}$ are opposite, [the previous $\left(V_{A}-V_{B}\right)$ shall be changed as $V_{A}+V_{B}$ and $\boldsymbol{F}_{l}$ is changed as the negative value]. Moreover, this formula is in line with the actual condition indeed obviously.

The above-mentioned $V_{A}$ and $V_{B}$ are the average velocities of movement of the free electron in the leads A and $\mathrm{B}$; the formula (9) shall be still valid if the analysis is implemented according to the actual motion situation of electron, as explained below:

Suppose that $I_{A}$ is composed of $\mathrm{m}$ groups of micro current beams $\Delta I_{A 1}, \Delta I_{A 2} \ldots \Delta I_{A m}$ having identical free

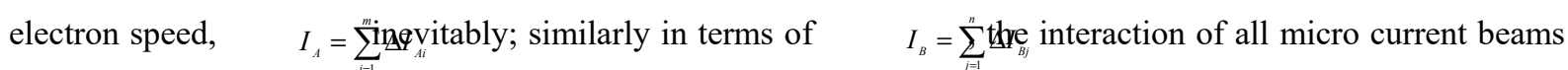
in $I_{A}$ and $I_{B}$ shall be in line with the Formula (9) because the interaction of any two micro current beams $\Delta I_{A i}$ and $\Delta I_{B j}$ in $I_{A}$ and $I_{B}$ are in line with the Formula (9).

$\boldsymbol{F}_{l}=\frac{\mu_{0} 2 l}{4 \pi r} \boldsymbol{Y}_{0} \sum_{i=1}^{m} \sum_{j=1}^{n} \Delta I_{i i} \Delta I_{B j} \quad$ shall be valid inevitably; while $\sum_{i=1}^{m} \sum_{j=1}^{n} \Delta I_{t i} \Delta I_{B j}=I_{A} I_{B}$, that is, the Formula (9) shall also be valid, namely, the uneven distribution of the velocities of the free electrons will not affect the Formula (9).

Seen from the derivation on the basis of the above-mentioned analysis, the relative speeds thereof shall be taken as the basis when we analyze the acting forces of all free electrons and protons. The previous 3.4 explains that this analysis result shall have nothing to do with the frame of reference where the observer is located. Therefore, magnetic field and force generated in two parallel wires with current and defects related to the observer in previous 2.8 shall not exist in our analysis above.

\subsection{Isokinetic motion of surface charge}

One infinite panel which is charged uniformly shall generate the electric field that is distributed uniformly; and the previous 3.2 points out the field strength $\mathrm{E}$ of the uniform electric field shall be increased as $E^{\prime}=E / \sqrt{1-V^{2} / C^{2}}$ inevitably when the uniform electric field moves at the speed $\mathrm{V}$ in any direction. In the reality, the usual rectangle cut-off coil and the rectangle permanent magnet we see can be taken as the surface charges moving along one fixed direction in the plane. As for the rectangle current-carrying coil, the four sides of the rectangle coil can be taken as the current panels; suppose that the rectangle coil is composed of $\mathrm{n}$ turns of lead, each panel shall have the current density of $n I$ (I indicates the current strength of each lead); as for the rectangle permanent magnet, the four surfaces thereof can be taken as four current panels, that is, be similar to the rectangle current-carrying coil. Moreover, the Lorentz force and the permanent magnet shall be analyzed according to the explanation of the above-mentioned current panel. 


\subsubsection{Analysis of Lorentz force}

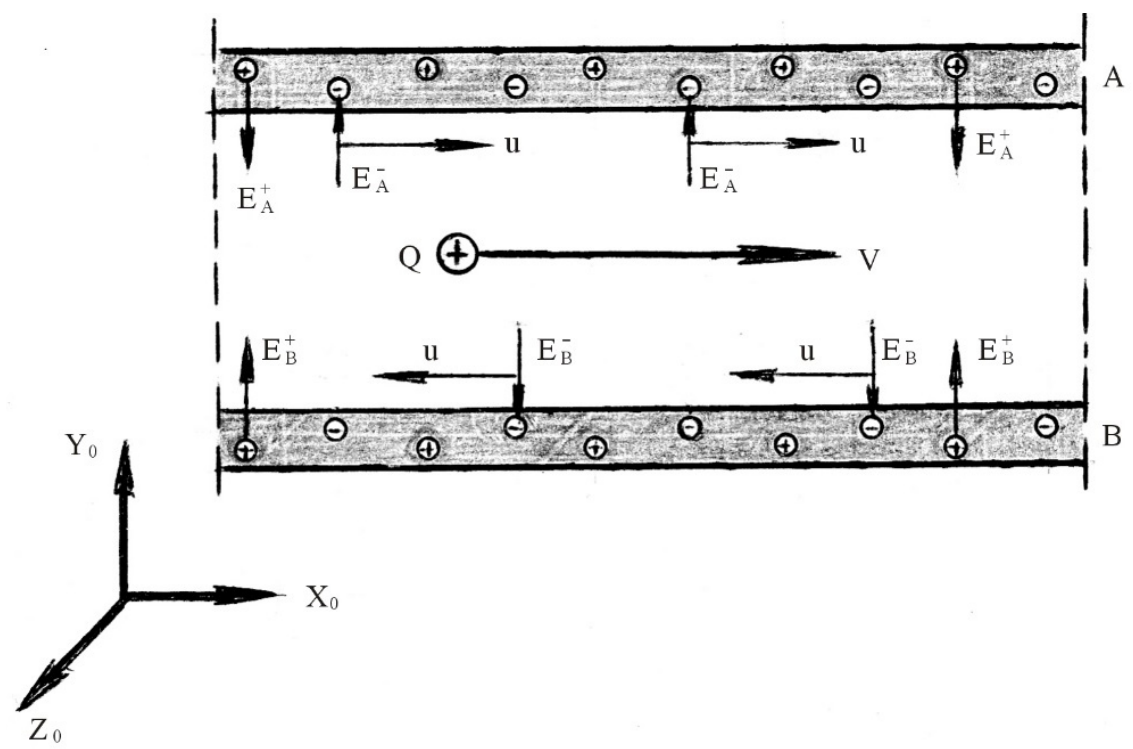

Figure 16.

A and B indicate two current surfaces composed of the infinite wires with currents; the two current surfaces of A and B shall be composed of the common wire with current, as shown in Figure 16, wherein the currents of A and $\mathrm{B}$ are equal and opposite in direction. $\boldsymbol{E}_{A}^{-}$and $\boldsymbol{E}_{B}^{-}$in the figure indicate the field intensities generated by the negative moving electrons in the current surface, respectively; while $\boldsymbol{E}_{A}^{+}$and $\boldsymbol{E}_{B}^{+}$indicate the field intensities generated by the positive static electrons having electricities equal to that of the negative electron, respectively.

The magnetic induction strength among two current surfaces of $\mathrm{A}$ and $\mathrm{B}$ should be acquired first; from the Formula (1) $\boldsymbol{B}=\mu_{0} \varepsilon_{0} \boldsymbol{V} \times \boldsymbol{E}, \mathrm{B}$ is from the moving electric field; motionlessness of $\boldsymbol{E}_{A}^{+}$and $\boldsymbol{E}_{B}^{+}$is related to B, and the $\boldsymbol{E}_{A}^{-}$electric field moving at the speed $\boldsymbol{u}$ and the $\boldsymbol{E}_{B}^{-}$electric field moving at the speed $-\boldsymbol{u}$ shall be analyzed. Moreover, $\boldsymbol{E}_{A}^{-}$and $\boldsymbol{E}_{B}^{-}$are equal and opposite in direction obviously, that is, $\boldsymbol{E}_{B}^{-}=-\boldsymbol{E}_{A}^{-}$, wherein E indicate the absolute values of $\boldsymbol{E}_{A}^{-}$and $\boldsymbol{E}_{B}^{-}$.

The magnetic induction strength $\boldsymbol{B}_{A}$ generated by $\boldsymbol{E}_{A}^{-}$shall be calculated; and from the Formula (1),

$$
\boldsymbol{B}_{A}=\mu_{0} \varepsilon_{0} \boldsymbol{u} \times \boldsymbol{E}_{A}^{-}=\mu_{0} \varepsilon_{0} u E \boldsymbol{Z}_{0}\left(\boldsymbol{Z}_{0} \text { refers to the unit vector in the direction } Z\right)
$$

The feeling strength $\boldsymbol{B}_{B}$ generated by $\boldsymbol{E}_{B}^{-}$shall be

$$
\boldsymbol{B}_{B}=\mu_{0} \varepsilon_{0}(-\boldsymbol{u}) \times \boldsymbol{E}_{B}^{-}=-\mu_{0} \varepsilon_{0} \boldsymbol{u}\left(-\boldsymbol{E}_{A}^{-}\right)=\mu_{0} \varepsilon_{0} u E \boldsymbol{Z}_{0}
$$

The total feeling strength generated by two current surfaces of A and B shall be:

$$
\boldsymbol{B}=\boldsymbol{B}_{A}+\boldsymbol{B}_{B}=2 \mu_{0} \varepsilon_{0} u E \boldsymbol{Z}_{0}
$$

$Q$ indicates that the particle having positive charge moves parallel with the two current surfaces at speed $V$, as shown in Figure 1. Moreover, the Lorentz force exerted to $Q$ according to the formula of Lorentz force in the field of electromagnetism should be:

$$
\boldsymbol{F}=Q \boldsymbol{V} \times \boldsymbol{B}=2 \mu_{0} \varepsilon_{0} V u E Q\left(-\boldsymbol{Y}_{0}\right) \quad\left(\boldsymbol{Y}_{0} \text { refers to the unit vector in the direction } Y\right)(10)
$$

The Formula (10) shall be the expression of electromagnetism for the Lorentz force in the presence of Figure 16. It should be started from the source of stress on $Q$ when we analyze the source of Lorentz force. The stress on $Q$ is exclusively because of action of the electric field unquestionably. Four electric fields including $\boldsymbol{E}_{A}^{+}, \boldsymbol{E}_{B}^{+}, \boldsymbol{E}_{A}^{-}$ and $\boldsymbol{E}_{B}^{-}$exist among two current surfaces of $\mathrm{A}$ and $\mathrm{B}$, but the acting forces of $\boldsymbol{E}_{A}^{+}$and $\boldsymbol{E}_{B}^{+}$to $Q$ are constant equal and opposite in direction; the effects thereof to $Q$ are not required to taken into account because the acting 
forces offset each other. Moreover, $\boldsymbol{E}_{A}^{-}$and $\boldsymbol{E}_{B}^{-}$shall be the only sources of the stress on $Q$ when $\boldsymbol{E}_{A}^{+}$and $\boldsymbol{E}_{B}^{+}$are excluded.

The relative speeds of $\boldsymbol{E}_{A}^{-}, \boldsymbol{E}_{B}^{-}$and $Q$ shall be confirmed before analyzing the interaction of $\boldsymbol{E}_{A}^{-} . \boldsymbol{E}_{B}^{-}$and $Q$ in accordance with the rule of relative velocity; and it can be seen that the relative speeds of $\boldsymbol{E}_{A}^{-}$and $Q$ are $V-u$ usually, and the relative speeds of $\boldsymbol{E}_{B}^{-}$and $Q$ are $V+u$. However, this kind of velocity addition method shall not be applicable when the velocity of motion of $Q$ is great; and the theory of relativity pointed out that the composition of velocities of the theory of relativity should be taken for composition of velocities of the objects moving at high speed. Our analysis conclusion should be applicable both for the object moving at low speed and the object moving at high speed in view of the principle of being applicable comprehensively. Therefore, we should calculate the relative velocity by using the composition of velocities of the theory of relativity.

The composition of velocities of the theory of relativity shall include the composition of velocities in directions $X, Y$ and $Z$. As for Figure 16, the speed in direction $Z$ does not exist; the extremely small migration velocity exists in the direction $Y$ and can be ignored. Therefore, the composition of velocities in the direction $X$ shall be analyzed, wherein the formula for composition of velocities in the direction $X$ shall be:

$$
V_{x}=\frac{u_{x}+\omega}{1+u_{x} \omega / C^{2}}
$$

In the formula, $\omega$ refers to the inertial system $Z$ which is static with respect to the object $M$, and by which the velocity of motion of another inertial system $Z^{\prime}$ can be observed; $u_{x}$ refers to the velocity of motion of object $N$ with respect to $Z$ '; $V x$ refers to the relative speeds of $M$ and $N$. As for the current surface of A in the Figure 16, the force exerted to $Q$ (object $M$ ) shall be acquired; $Q$ belongs to the inertial system $Z$, and A belongs to the inertial system $Z^{\prime}$; and the velocity of motion of the $Z^{\prime}$ (A and B) is $-V$ from $Z$, that is, $\omega=-V$ in the composition formula; the velocity of motion of $\boldsymbol{E}_{A}^{-}$(object $\mathrm{N}$ ) is $u$ from $Z^{\prime}$, that is, $u_{x}=u$ in the composition formula. Therefore, the velocity of motion of $Q$ with respect to $\boldsymbol{E}_{A}^{-}$should be:

$$
V_{A}=\frac{u-V}{1+u V / C^{2}}
$$

Similarly, the velocity of motion of $Q$ with respect to $\boldsymbol{E}_{B}^{-}$should be:

$$
V_{B}=\frac{-u-V}{1+u V / C^{2}}
$$

Een from $Q, \boldsymbol{E}_{\mathrm{A}}^{-}$moving at speed $\mathrm{V}$ should be contracted and increased as

$$
\begin{aligned}
& \boldsymbol{E}_{A}^{-^{\prime}}=\boldsymbol{E}_{A}^{-} / \sqrt{1-V_{A}^{2} / C^{2}} \\
& =\frac{\boldsymbol{E}_{A}^{-}}{\sqrt{1-\left(\frac{u-V}{1-u V / C^{2}}\right)^{2} / C^{2}}}=\frac{\boldsymbol{E}_{A}^{-}}{\sqrt{1-\frac{C^{4}(u-V)^{2}}{\left(C^{2}-u V\right)^{2}}} / C^{2}} \\
& =\frac{\left(C^{2}-u V\right) \boldsymbol{E}_{A}^{-}}{\sqrt{\left(C^{2}-u V\right)^{2} C^{2}(u-V)^{2}}}=\frac{\left(C^{2}-u V\right) \boldsymbol{E}_{A}^{-}}{\sqrt{C^{4}+u^{2} V^{2}-C^{2} u^{2}-C^{2} V^{2}}} \\
& =\frac{\left(C^{2}-u V\right) \boldsymbol{E}_{A}^{-}}{\sqrt{\left(C^{2}-V^{2}\right)\left(C^{2}-u^{2}\right)}}=\frac{\left(C^{2}-u V\right) \boldsymbol{E}_{A}^{-}}{C^{2} \sqrt{\left(1-V^{2} / C^{2}\right)\left(1-u^{2} / C^{2}\right)}}
\end{aligned}
$$


Because the velocities $u$ of the negative electrons of the two current surfaces of $A B$ are far smaller than $C$ (the revolving speed of the electron of magnetic domain is also far smaller than $\mathrm{C}$ ), $u^{2} / C^{2}$ is very small, and it can be considered that $1-u^{2} / C^{2} \approx 1$, the formula above can be simplified as:

$$
\boldsymbol{E}_{A}^{-}=\frac{\left(C^{2}-u V\right) \boldsymbol{E}_{A}^{-}}{C^{2} \sqrt{1-V^{2} / C^{2}}}
$$

$\boldsymbol{E}_{B}^{-}$moving at speed $V$ should be contracted and increased as: $\boldsymbol{E}_{B}^{-}=\boldsymbol{E}_{B}^{-} / \sqrt{1-V_{B}^{2} / C^{2}}$; and when the previous $V_{B}$ is substituted, the formula below can be acquired:

$$
\begin{aligned}
\boldsymbol{E}_{B}^{-}= & \frac{\boldsymbol{E}_{B}^{-}}{\sqrt{1-\left(\frac{u+V}{1+u V / C^{2}}\right)^{2} / C^{2}}}=\frac{\boldsymbol{E}_{B}^{-}}{\sqrt{1-\frac{C^{4}(u+V)^{2}}{\left(C^{2}+u V\right)^{2}}} / C^{2}} \\
& =\frac{\left(C^{2}+u V\right) \boldsymbol{E}_{B}^{-}}{\sqrt{\left(C^{2}+u V\right)^{2}-C^{2}(u+V)^{2}}}=\frac{\left(C^{2}+u V\right) \boldsymbol{E}_{B}^{-}}{\sqrt{C^{4}+u^{2} V^{2}-C^{2} u^{2}-C^{2} V^{2}}} \\
& =\frac{\left(C^{2}+u V\right) \boldsymbol{E}_{B}^{-}}{\sqrt{\left(C^{2}-V^{2}\right)\left(C^{2}-u^{2}\right)}}=\frac{\left(C^{2}+u V\right) \boldsymbol{E}_{B}^{-}}{C^{2} \sqrt{1-V^{2} / C^{2}}}
\end{aligned}
$$

The total field strength felt by $Q$ should be

$$
\boldsymbol{E}^{\prime}=\boldsymbol{E}_{B}^{\prime}+\boldsymbol{E}_{A}^{\prime}=\frac{\left(C^{2}+u V\right) \boldsymbol{E}_{\mathrm{B}}^{\prime}}{C^{2} \sqrt{1-V^{2} / C^{2}}}+\frac{\left(C^{2}-u V\right) \boldsymbol{E}_{A}^{-}}{C^{2} \sqrt{1-V^{2} / C^{2}}}
$$

Because $\boldsymbol{E}_{A}^{--}=-\boldsymbol{E}_{B}^{-}$, the formula:

$$
\boldsymbol{E}^{\prime}=\frac{2 u V \boldsymbol{E}_{B}^{-}}{C^{2} \sqrt{1-V^{2} / C^{2}}}=\frac{2 \mu_{0} \varepsilon_{0} u V E}{\sqrt{1-V^{2} / C^{2}}}\left(-\boldsymbol{Y}_{0}\right) \quad \text { (11) can be obtained upon }
$$

simplification after substituting $\boldsymbol{E}_{A}^{\prime-}=-\boldsymbol{E}_{B}^{-}$into the formula above.

The force exerted to $Q$ when moving at high speed in the magnetic field shall be indicated by using $\boldsymbol{F}_{V}$ in order to distinguish the acting force $\boldsymbol{F}$, that is, Lorentz force defined electromagnetically of $Q$ when moving at low speed in the magnetic field, and the formula below can be acquired from the Formula (11):

$$
\boldsymbol{F}_{V}=\boldsymbol{E}^{\prime} Q=\frac{2 \mu_{0} \varepsilon_{0} u V E Q}{\sqrt{1-V^{2} / C^{2}}}\left(-\boldsymbol{Y}_{0}\right)
$$

It can be seen after comparing this formula with the Formula (10) of Lorentz force that:

$$
\boldsymbol{F}_{V}=\frac{\boldsymbol{F}}{\sqrt{1-V^{2} / C^{2}}}
$$

$\sqrt{1-V^{2} / C^{2}}=1$ and $\boldsymbol{F}_{V}=\boldsymbol{F}$ shall be available obviously when the velocity of motion $V$ of $Q$ is small, that is, $\boldsymbol{F}_{V}$ is Lorentz force when $Q$ moves at low speed, that is, $\boldsymbol{F}_{V}$ can be taken as the modifier formula of Lorentz force.

The deflection result of the charged particle moving at high speed in the magnetic field can be deduced by using the Formula (13). Moreover, the deflection distance of the charged particle in the magnetic field should be as follows according to the test when the velocity of motion of the charged particle is not great:

$$
Y=\frac{1}{2} a t^{2}=\frac{F_{V}}{2 m} t^{2}=\frac{F}{2 m} t^{2}
$$


$m$ should be transformed as $m^{\prime}=m / \sqrt{1-V^{2} / C^{2}}, t$ should be transformed as $t^{\prime}=t \sqrt{1-V^{2} / C^{2}}$ according to the special relativity when the velocity of motion of the charged particle is great. The force $\boldsymbol{F}_{V}$ shall move along with $Q$, and $F_{V}^{\prime}=F_{V} / \sqrt{1-V^{2} / C^{2}}\left(\cos 90^{\circ}=0\right)$ shall be available according to the Formula (4) of force transformation in 3.3. Moreover, the Formula (14) below can be acquired in presence of $F_{V}^{\prime}=F /\left(1-V^{2} / C^{2}\right)$ after substituting $m^{\prime}, t^{\prime}$ and $F_{V}^{\prime}$ which must be transformed to the formula above because $F_{V}=F / \sqrt{1-V^{2} / C^{2}}[$ Formula (13)] when $V$ is very great.

$$
Y=\frac{F_{V}^{\prime} t^{\prime 2}}{2 m^{\prime}} t^{2}=\frac{F /\left(1-V^{2} / C^{2}\right)}{2 m / \sqrt{1-V^{2} / C^{2}}} t^{2}\left(1-V^{2} / C^{2}\right)=\frac{F t^{2}}{2 m} \sqrt{1-V^{2} / C^{2}}
$$

In the formula, $F=B Q V$ is taken as the Lorentz force; and the Formula (14) is in line with the test result obviously. However, it is not fully proved only by using the Formula (14) because the Formula (14) is the analysis result obtained by the observer who is static with respect to the magnetic field (two current surfaces in Figure 16); while the observer moving with $Q$ at the identical speed shall have the identical analysis result according to the relativity principle; therefore, we should analyze the deflection of the charged particle as the observer moving at the speed $V$.

Seen from the observer moving at the speed $V, Q$ does not move in the direction $X$, and moves slightly in the direction $Y$; therefore, the mass $m$ of $Q$ is not transformed. $Q$ is subjected to the acting force $F_{V}$ when the two current surfaces pass through $Q$ at the speed $-V$, wherein the $F_{V}$ shall be static and not transformed with respect to the $Q$. Moreover, the observer deduces that displacement of $Q$ in the direction $Y$ must be

$Y=\frac{1}{2} \cdot \frac{F_{V}}{m} t_{V}^{2}$ after measuring the time $t_{V}$ during which $Q$ passed through the magnetic field.

What was the relationship between $t_{V}$ and the $t$ measured by the observer who was static with respect to the magnetic field? It was known that the measuring speeds of the two observers were equal, but the widths of the magnetic field that the two observers saw were different, wherein the width of the static magnetic field saw by the former was $L$, and the time during which the particle passed through $L$ was $t=L / V$; while the later saw the magnetic field moved at the speed $-V$, therefore the width of the magnetic field was $L^{\prime}=L \sqrt{1-V^{2} / C^{2}}$, and time was $t_{r}=L^{\prime} / V=t \sqrt{1-V^{2} / C^{2}}$, and $Y=\frac{F t^{2}}{2 m} \sqrt{1-V^{2} / C^{2}}$ shall be available when $F_{V}=F / \sqrt{1-V^{2} / C^{2}}$ are $t_{V}=t \sqrt{1-V^{2} / C^{2}}$ substituted into the formula above.

This result is identical with the Formula (14) obviously. Thus, the deflection result of the high-speed charged particle has been proved fully at this point.

What should be pointed out is that $F=B Q V$ of Lorentz force shall be applicable for the object moving at low speed in the uniform magnetic filed, and the $F_{V}$ modified of Lorentz force shall be applicable for the uniform magnetic field as well. Neither $F$ nor $F_{V}$ shall be applicable for the inhomogeneous magnetic field. Moreover, neither $F$ nor $F_{V}$ can solve the problems that the acting force and the reacting force as shown in Figure 2 in previous 2.6 are unequal but the same in direction.

\subsubsection{Electric Field around Permanent Magnet}

It is pointed out in the section about interaction of two parallel wires with current in 4.2.1 that the total field strength of the lead A and exerted to the static $d Q_{+}$shall be $\boldsymbol{E}_{O_{+}}=K \tau_{A}^{+} V_{A}^{2} \boldsymbol{Y}_{0} / C^{2} r$, that is, the static negative electric field ( $\boldsymbol{Y}_{0}$ points to the lead A) exists around the static wire A with current. Can this electric field be detected by using the test? It is proved by calculation that this electric field is extremely weak and namely cannot be measured; taking the copper wire with section area of $100 \mathrm{~mm}^{2}$ as an example, the negative field strength at the place $10 \mathrm{~mm}$ far from the lead shall be $7.387 \times 10^{-6} \mathrm{~V} / \mathrm{m}$ only when the lead is charged with the extremely great current of $1000 \mathrm{~A}$ on the basis that each copper atom should have one free electron, which is equivalent to the field strength among two plates when the two infinite metal plates with distance of $200 \mathrm{~km}$ are connected with one $1.5 \mathrm{~V}$ battery and cannot be measured naturally (the force of the wire $\mathrm{B}$ with current is obvious because the amounts of electricity of the free electron and the proton in B are great).

It is found by analyzing the above-mentioned $E_{Q+}$ that $\boldsymbol{E}_{Q_{+}}=K I_{A} V_{A} Y_{0} / C^{2} r_{0}$ can be acquired after substituting $V_{A} \tau_{A}^{+}=I_{A}$ into this formula; thus, the $E_{Q_{+}}$may be measured if $V_{A}$ is great on the premise that $I_{A}$ is not changed. $V_{A}$ should not be great in the lead certainly; but the strong $E$ may exist certainly because the velocity 
of automatic rotation of the electron is great and is about $10^{6} \mathrm{~m} / \mathrm{s}$ usually in the magnetic domain of permanent magnet.

I implemented measurement taking the cube $\mathrm{NdFeB}$ permanent magnet with the surface feeling strength about $0.8 \mathrm{~T}$ and side of $20 \mathrm{~mm}$ long as the test sample in 2004 . We can take one turn of rectangle current-carrying coil composed of the square surfaces with uniform surface current and side of $20 \mathrm{~mm}$ long on each surface as the analysis model because the magnetic field generated by this test sample was similar to the magnetic field having current strength of 29209A and generated by one turn of rectangle coil.

It is learnt from the analysis by taking each surface of the above-mentioned model as one section of wire with current in presence with the method of analyzing the moving electric field of the wire with current in 4.2.1 that the field strength components of the moving electric fields generated by motion (current) of negative electrons on four surfaces of the model are equal in the direction of axis of NS pole; therefore, the field strength on the axis of NS pole is the sum of the negative field intensities on four surfaces, and the negative field strength increased by resultant motion is greater than the positive field intensities of the motionless protons on the four sides obviously, and the effect of negative electric field shall be shown due to composition thereof. However, the electric field cannot be measured at the place near the axis of NS pole after more than one hundred times of tests and measurements.

Thus, the deduction is incorrect obviously. The writer did not find the reason for a long time, and acquired by subsequent analysis that the states of motion of two electric fields which have identical direction and move oppositely shall be offset according to the rule in previous 3.6. It is learnt from the rule that the negative moving electric field generated on any current surface of the permanent magnet must have the component which is equal and opposite to that of the negative moving electric field on the opposite current surface, the states of motion thereof must be offset each other. Therefore, the electric field cannot be measured at the place near the NS axis.

The moving electric field shall not be offset completely when not located in the other space outside the N.S axis according to the analysis; and the great total field strength may exist in certain space. Moreover, the condition of respective moving electric fields shall be analyzed specifically in the direction parallel with the $\mathrm{N} \cdot \mathrm{S}$ axis (the direction $\mathrm{B}$ of feeling strength), the direction vertical to the $\mathrm{N} \cdot \mathrm{S}$ axis and the direction vertical to the center of the current surface.

4.3.2.1 Calculation of Positive Field Strength and Negative Field Strength in N·S Direction in Front of the Coil

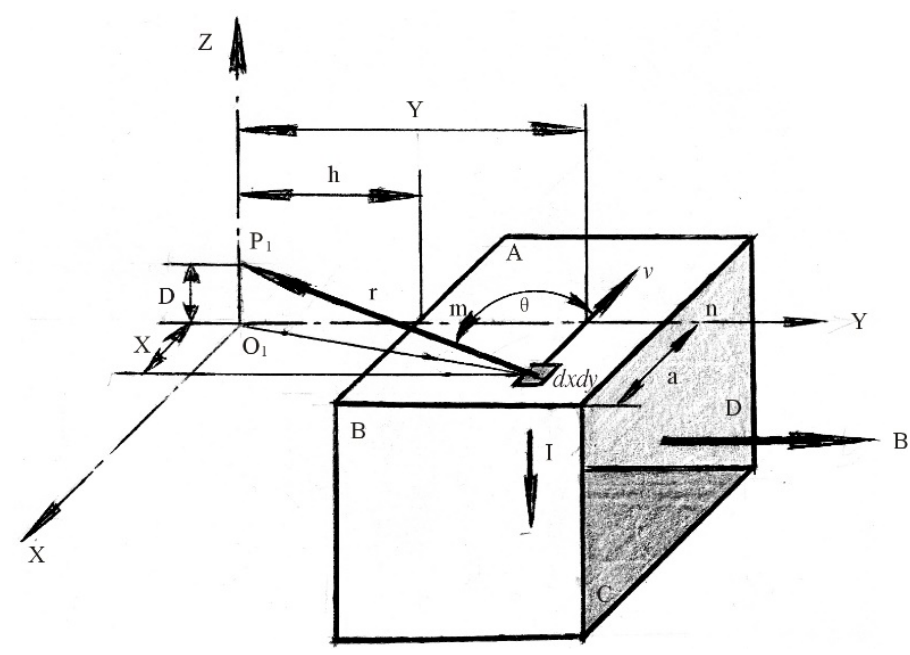

Figure 17. Total field strength on $\mathrm{N} \cdot \mathrm{S}$ direction in front of coil

$\mathrm{A}, \mathrm{B}, \mathrm{C}$ and $\mathrm{D}$ indicate the analysis model of square current surfaces having sides of $2 \alpha$ long, as shown in Figure 17. One turn of rectangle coil is formed by the four current surfaces and shall be identical with the permanent magnet having identical shape and surface feeling strength of $0.8 \mathrm{~T}$ when the current of the coil is 29209A, 
wherein the direction of the current of the coil in the figure is vertical to $y$, the direction of the magnetic field at the center of the coil is parallel with $\mathrm{y}$; y passes through the central line $\mathrm{mn}$ of the surface $\mathrm{A} ; \mathrm{h}$ indicates $\mathrm{O}_{1} \mathrm{~m}$, and $\mathrm{h}_{1}=5 \times 10^{-3} \mathrm{~m}$ and $\mathrm{h}_{2}=10^{-2} \mathrm{~m}$ shall be taken during test; $\mathrm{D}$ indicates $\mathrm{O}_{1} \mathrm{p}$, and $\mathrm{D}=2 \times 10^{-3} \mathrm{~m}$ shall be taken during test. And now, the total field strength of the coil, that is, the electrons and the protons on four surfaces A, B, C and $\mathrm{D}$, in the direction $\mathrm{y}$ of point $\mathrm{P}_{1}$ shall be acquired.

First see the surface B and surface D. The field intensities of the moving electric fields generated during motion of the electrons on the surface $\mathrm{B}$ and the surface $\mathrm{D}$ are equal and identical in direction in the direction $\mathrm{y}$ of the point $\mathrm{P}_{1}$, while the directions of motion thereof are opposite (the directions of two currents are opposite), the states of motion are offset each other, and the negative electric fields of the two are equivalent to the static and negative electric field. Moreover, the surface B and the surface D shall not be taken into account, and we shall analyze the total field intensities of the surface $A$ and the surface $C$ respectively because the total field strength of the negative electric field and the positive electric field (generated by the proton having electricity equal to that of the moving electron) is 0 in the direction y of the point $P_{1}$.

$\sigma_{+}$and $\sigma_{-}$indicate the surface charge density of the moving electrons on all surfaces of the coil and the surface charge density of the proton having electricity equal to that of the moving electrons respectively; and $\sigma_{-}=-\sigma_{+}$obviously. If $v$ indicates the velocity of motion of the electron, $\sigma_{-}=-I / 2 a v$. Moreover, the field intensities of the protons having electricity equal to that of the moving electrons on the surface $\mathrm{A}$ shall be analyzed in the direction $y$ of the point $\mathrm{P}_{1}$ first.

Electricity of the protons having the electricity equal to that of the moving electrons in the micro surface current element $\mathrm{dxdy}$ of point $\mathrm{j}$ shall be $d Q_{+}=\sigma_{+} d x d y$, as shown in the figure, and the field strength of $d Q_{+}$in the direction $\mathrm{y}$ of the point $\mathrm{P}_{1}$ first shall be:

$$
d E_{A 1}^{+}=K \frac{d Q}{r^{2}} \frac{y}{r}=\frac{k \sigma_{+} y d x d y}{r^{3}}
$$

From the figure, $r=\sqrt{x^{2}+y^{2}+D^{2}}$

$$
\begin{aligned}
E_{A 1}^{+} & =\iint d E_{A 1}^{+}=K \sigma_{+} \int_{h}^{h+2 a} y a y \int_{-a}^{+a} \frac{d x}{\left(x^{2}+y^{2}+D^{2}\right)^{3 / 2}} \\
& =2 K \sigma_{+} \int_{h}^{h+2 a} \frac{a y d y}{\left(D^{2}+y^{2}\right) \sqrt{D^{2}+y^{2}+a^{2}}} \\
& =2 K \sigma_{+}\left[\ln \frac{\sqrt{D^{2}+y^{2}}}{a+\sqrt{D^{2}+y^{2}+a^{2}}}\right]_{h}^{h+2 a}
\end{aligned}
$$

It is known that $\alpha=10^{-2} \mathrm{~m}$ and $\mathrm{D}=2 \times 10^{-3} \mathrm{~m}$, and $E_{A 1}^{+}=2 \mathrm{k} \sigma_{+} \times 0.988917594 \mathrm{~V} / \mathrm{m} \quad$ and $E_{A 2}^{+}=2 k \sigma_{+} \times 0.540825494 \mathrm{~V} / \mathrm{m}$ shall be available when calculating the $\mathrm{h}=5 \times 10^{-3} \mathrm{~m}$ and $\mathrm{h}=10^{-2} \mathrm{~m}$ according the Formula (15).

As for point $\mathrm{P}_{1}$, D of the surface A shall be increased as D+2a. The Formula shall be also applicable for $E_{C}^{+}$to calculate the surface $\mathrm{C}$, but, $\mathrm{D}$ should be replaced by D+2a. Moreover, $E_{\mathrm{Cl}}^{+}=2 \mathrm{~K} \sigma_{+} \times 0.133932912 \mathrm{~V} / \mathrm{m}$ and $E_{C 2}^{+}=2 K \sigma_{+} \times 0.137154744 \mathrm{~V} / \mathrm{m}$ shall be available during calculating and acquiring $\mathrm{h}=5 \times 10^{-3} \mathrm{~m}$ and $\mathrm{h}=10^{-2} \mathrm{~m}$ by substituting D+2a into the Formula (15).

Then the field intensities of the moving electric fields generated during motion of the electrons of the surface A and the surface $\mathrm{C}$ in the direction y of point $\mathrm{P}_{1}$ shall be analyzed, and the field strength $E_{A 1}^{-}$generated on the surface A should be analyzed first. Moreover, the previous 1 indicates that the relationship between the field strength $\mathrm{E}$ at the place $\mathrm{r}$ outside $\mathrm{Q}$ and the field strength $E^{\prime}$ of $\mathrm{Q}$ at the place $\mathrm{r}$ after moving at the speed $v$ shall be:

$E^{\prime}=K \frac{Q}{r^{2}}\left(1-v^{2} / C^{2}\right) /\left(1-\sin ^{2} \theta v^{2} / C^{2}\right)^{3 / 2}$. Therefore, $d E_{A 1}^{-}$of the field strength generated by motion of the 
electrons at the point $\mathrm{x}$ and $\mathrm{y}$ in the Figure 3 shall be:

$$
d E_{A 1}^{-}=\frac{k \sigma_{-} y d x d y}{r^{3}} \frac{\left(1-v^{2} / C^{2}\right)}{\left(1-\sin ^{2} \theta v^{2} / C^{2}\right)^{3 / 2}}
$$

In the formula, $\theta$ indicates the included angle formed by $v$ and $\mathrm{r}$; from the figure, $\sin \theta=\sqrt{D^{2}+y^{2}} / r$; the formula below can be acquired after simplification if the relation is substituted into the formula $d E_{A 1}^{-}$and order $n=\sqrt{1-v^{2} / c^{2}}$ :

$$
\begin{gathered}
d E_{A 1}^{-}=\frac{k \sigma_{-} n^{2} y d y d x}{\left[x^{2}+\left(D^{2}+y^{2}\right) n^{2}\right]^{3 / 2}} \\
E_{A 1}^{-}=\iint d E_{A 1}^{-}=2 K \sigma_{-}\left[\ln \frac{\sqrt{D^{2}+y^{2}}}{\frac{a}{n}+\sqrt{\left(D^{2}+y^{2}\right)+\frac{a^{2}}{n^{2}}}}\right]_{n}^{n+2 a}
\end{gathered}
$$

The relation of $E_{C I}^{-}$is similar to the relation (16) of $E_{A 1}^{-}$; and the $D^{2}$ in the (16) is replaced by $(D+2 \alpha)^{2}$ only.

The total field strength of the surface A at the point $P_{1}$ refers to the composition of the negative moving electric field $E_{A 1}^{-}$and the electric field $E_{A 1}^{+}$of the positive proton (having electricity equal to the electron), the direction of $E_{A 1}^{-}$is opposite to that of $E_{A 1}^{+}$, and the total field strength shall be $E_{A 1}=E_{A 1}^{-}-E_{A 1}^{+}$. In order to facilitate understanding, the values of this formula and the formula below for analyzing the field strength $E$ shall be the absolute values of the field strength $E$. Similarly, the total field strength of $E_{C 1}^{-}$and $E_{C 1}^{+}$on the surface $C$ shall be $E_{C 1}=E_{C 1}^{-}-E_{C 1}^{+} . E_{A 1}$ and $E_{C 1}$ are the moving electric fields, but the directions of motion are opposite; one part of moving electric field of $E_{A 1}$ shall be offset by $E_{C 1}$. Moreover, the total field strength of the two shall be $E_{1}=E_{A 1}-E_{C 1}$; and the formula $E_{1}=\left(E_{A 1}^{-}-E_{A 1}^{+}\right)-\left(E_{C 1}^{-}-E_{C 1}^{+}\right)=\left(E_{A 1}^{-}-E_{C 1}^{-}\right)-\left(E_{A 1}^{+}-E_{C 1}^{+}\right)$(17) can be acquired after substituting $E_{A 1}=E_{A 1}^{-}-E_{A 1}^{+}$and $E_{C 1}=E_{C 1}^{-}-E_{C 1}^{+}$into this formula.

Order E2 indicates $\mathrm{h}$ in the Formula (15) and Formula (16); and the total field strength shall be $E_{2}=\left(E_{A 2}^{-}-E_{C 2}^{-}\right)-\left(E_{A 2}^{+}-E_{C 2}^{+}\right)$when the $h_{1}=5 \times 10^{-3} \mathrm{~m}$ is changed as $h_{2}=10^{-2} \mathrm{~m}$.

\subsubsection{Total Field Strength on Side of the Coil Vertical to $N \cdot S$ Direction}

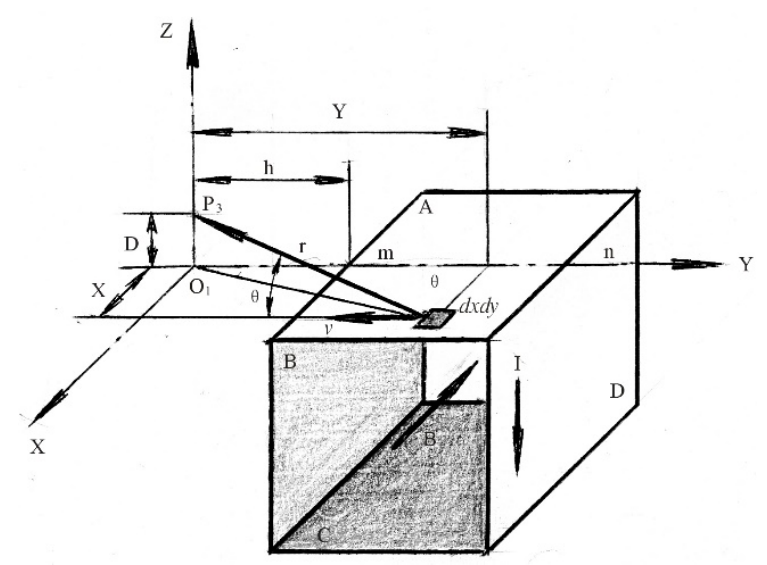

Figure 18. Total field strength on side of the coil vertical to $N \cdot S$ direction

The direction of motion of the electron on surface A of the coil is parallel with $O Y$, as shown in Figure 18; therefore, the included angle $\theta$ formed by the direction of motion of the electron and the direction (the direction $-r$ ) of field strength of the electron at the place $P_{3}$ is small usually; the previous 4.1 points out that $E^{\prime}<E$ when $\theta<54.7^{\circ}$. Therefore, $E_{A 3}^{-}<E_{A 3}^{+}$and $E_{c 3}^{-}<E_{c 3}^{+}$shall be available under this condition. As for $P_{3}$, 
the complete offset effect will not occur in the surface B and the surface D, which is similar to the surface A and the surface $\mathrm{C}$ after analyzing the surface $\mathrm{B}$ and the surface $\mathrm{D}$ of the Figure 18 ; and $E_{B 3}^{-}<E_{B 3}^{+}$and $E_{D 3}^{-}<E_{D 3}^{+}$ shall be available because the included angle formed by the speeds of all points and the field strength on the surface $\mathrm{B}$ and the surface $\mathrm{D}$ is also relatively small. The total field intensities of the surface $\mathrm{A}$ and the surface $\mathrm{C}$ should be $E_{3}^{A C}=\left(E_{A 3}^{+}-E_{C 3}^{+}\right)-\left(E_{A 3}^{-}-E_{C 3}^{-}\right)$, and the total field intensities of the surface B and the surface D should be $E_{3}^{B D}=\left(E_{B 3}^{+}-E_{D 3}^{+}\right)-\left(E_{B 3}^{-}-E_{D 3}^{-}\right)$after referring to the Formula (17) and the Formula (18). Moreover, the total field intensities of the four surfaces $\mathrm{A}, \mathrm{B}, \mathrm{C}$ and $\mathrm{D}$ shall be $E_{3}=E_{3}^{A C}+E_{3}^{B D}=\left(E_{A 3}^{+}-E_{C 3}^{+}\right)-\left(E_{A 3}^{-}-E_{C}^{-}\right)+\left(E_{B 3}^{+}-E_{D 3}^{+}\right)-\left(E_{B 3}^{-}-E_{D 3}^{-}\right)$because the directions of motions of the electrons on the surfaces $A$ and the surface $C$ are vertical to those of the surface $B$ and the surface $D$, and $E_{3}^{A C}$ will not be offset by $E_{3}^{B D}$.

The total field intensities of the four surfaces A, B, C and D shall be $E_{3}$ when $h=5 \times 10^{-3} \mathrm{~m}$ and $E_{4}$ when $h_{2}=10^{-2} \mathrm{~m}$.

The positions of the surface $\mathrm{A}$ and the surface $\mathrm{C}$ of the two are the same after comparing the Figure 17 and the Figure 18; therefore, $E_{A 3}^{+}=E_{A 1}^{+}, E_{C 3}^{+}=E_{c 1}^{+}$and $E_{A 4}^{+}=E_{A 2}^{+}$shall be available; the calculation formula of $E_{C 4}^{+}=E_{C 2}^{+}$for $E_{B 3}^{+}, E_{B 4}^{+}, E_{D 3}^{+}$and $E_{D 4}^{+}$, we will not introduce the deducing process (see the deducing method of the surface $\mathrm{A}$ and the surface $\mathrm{C}$ for reference) specifically, but only explain the deducing result:

$$
E_{B 3}^{+}=2 K \sigma_{+}\left[\sin ^{-1}\left(\sqrt{\frac{a^{2}}{a^{2}+h^{2}}} \sin \operatorname{tg}^{-1} \frac{z}{h}\right)\right]_{D}^{D+2 a}
$$

$E_{B 4}^{+}$can be acquired after changing $h=5 \times 10^{-3} \mathrm{~m}$ of the Formula (19) as $h_{2}=10^{-2} \mathrm{~m} ; E_{D 3}^{+}\left(h=5 \times 10^{-3} \mathrm{~m}\right)$ and $E_{D 4}^{+}\left(h=10^{-2} m\right)$ can be acquired by changing the $h$ of the Formula (19) as $2 a+h$.

The deducing result of $E_{A 3}^{-}$shall be:

$$
E_{A 3}^{-}=2 K \sigma_{-} n\left[\ln \frac{\sqrt{D^{2} n^{2}+y^{2}}}{a n+\sqrt{y^{2}+n^{2}\left(a^{2}+D^{2}\right)}}\right]_{h}^{h+2 a}
$$

The deducing result of $E_{B 3}^{-}$shall be:

$$
E_{B 3}^{-}=2 K \sigma_{-}\left[\sin ^{-1}\left(\sqrt{\frac{a^{2}}{a^{2}+h^{2}}} \sin \operatorname{tg}^{-1} \frac{z}{h n}\right)\right]_{D}^{D+2 a}
$$

$E_{A 4}^{-}$and $E_{D 4}^{-}$can be acquired by changing $h=5 \times 10^{-3} \mathrm{~m}$ in Formula (20) and the Formula (21) as $h=10^{-2} \mathrm{~m}$.

\subsubsection{Total Field Strength in Central Direction of Side of Coil}

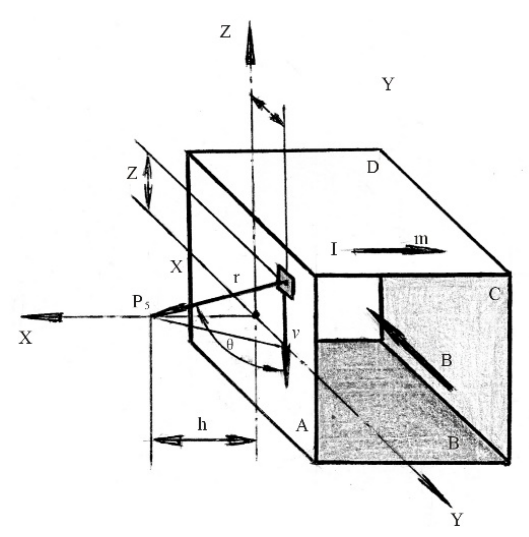

Figure 19. Analysis of field strength at center of side of the coil 
The NS direction of the coil is parallel with OY, and the direction of motion of the electron of the surface A is parallel with OZ, as shown in Figure 19. And now, the total field strength of point $P_{5}$ in the direction $\mathrm{X}$ shall be acquired.

The direction of the central magnetic field of the coil is parallel with Y, and $h$ indicates the distance between the point $P_{5}$ and the side A of the coil, as shown in Figure 19; and the origin $\mathrm{O}$ of coordinates is located at the center of the surface A. and now, the total field strength of point $P_{5}$ in the direction $\mathrm{X}$ shall be acquired.

From the figure, the surface $\mathrm{B}$ and the surface $\mathrm{D}$ are symmetrical around $P_{5}$, while the directions of the currents thereof are opposite; therefore, the moving electric fields of the moving electrons of the surface $\mathrm{B}$ and the surface D generated in the direction $\mathrm{X}$ of the point $P_{5}$ are equal, the directions thereof are identical, but the directions of motion thereof are opposite; the moving electric fields are offset completely and equivalent to the static negative electric field; therefore, the total field intensities of the electrons and the protons on the surface $\mathrm{B}$ and the surface $\mathrm{D}$ in the direction $\mathrm{X}$ of the point $P_{5}$ shall not be taken into account. Moreover, the total field intensities on the surface $\mathrm{A}$ and the surface $\mathrm{C}$ at the point $P_{5}$ shall be analyzed.

The deducing result of $E_{A 5}^{+}$shall be:

$$
E_{A S}^{+}=2 K \sigma_{+}\left[\sin ^{-1}\left(\sqrt{\frac{a^{2}}{a^{2}+h^{2}}} \sin \operatorname{tg}^{-1} \frac{z}{h}\right)\right]_{-a}^{+a}
$$

The deducing result of $E_{A 5}^{-}$shall be:

$$
E_{A 5}^{-}=2 K \sigma_{+}\left[\sin ^{-1}\left(\sqrt{\frac{a^{2}}{a^{2}+h^{2}}} \sin \operatorname{tg}^{-1} \frac{z}{h}\right)\right]_{-a}^{+a}
$$

$E_{A 6}^{+}$and $E_{A 6}^{-}$can be acquired when $h=5 \times 10^{-3} \mathrm{~m}$ of $E_{A 5}^{+}$and $E_{A 5}^{-}$is changed as $h=10^{-2} \mathrm{~m}$.

4.3.2.3 Experimental Method and Experimental Result

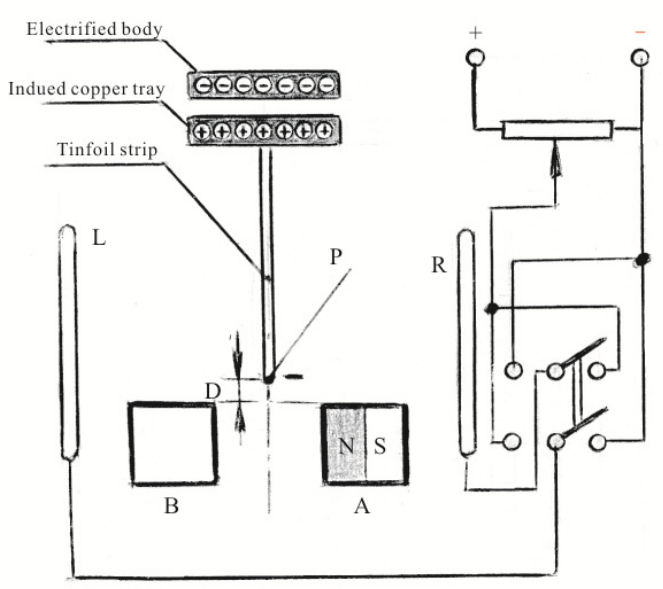

(a)

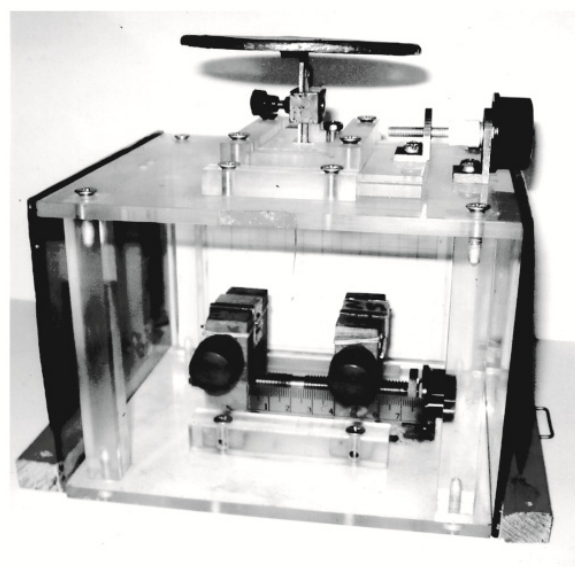

(b)

Figure 20. Schematic diagram of experimental method and physical photo

The schematic diagram of the test method is shown the Figure 20 (a), wherein A indicates the cube NdFeB permanent magnet with side of $20 \mathrm{~mm}$ long, and the magnetic induction strength on the surface thereof is about $0.8 \mathrm{~T}$; $\mathrm{B}$ is the same as the $\mathrm{A}$ in terms of dimensions and made from NdFeB; but B is not magnetized and not the 
permanent magnet. D refers to the induction copper disc for making the bottom end $\mathrm{P}$ of the tin foil strip underneath feel the charge which is the same as the charged body by receiving the electrostatic induction of the charged body; and $\mathrm{D}$ shall be moveable from the left side to the right side, forwards and backward by means of adjustment; the tin foil strip can move up and down, therefore $\mathrm{P}$ can be positioned at any position among $\mathrm{A}$ and $\mathrm{B}$. $\mathrm{L}$ and $\mathrm{R}$ are the planar metal plates having great areas and can generate uniform electric field in the middle area there between after being connected with the DC power supply. The direction of the electric field among $\mathrm{L}$ and $\mathrm{R}$ shall be changed when the direction of the change-over switch is changed. Moreover, the photo of the physical experimental device is shown in the Figure (20).

The charged tin foil strip P shall be attracted by the permanent magnet inevitably because of the effect of electrostatic induction; therefore, the measuring test of the electric field cannot be implemented. In order to eliminate the electrostatic induction, the experimental device shall be installed at the left end of the tin foil strip $\mathrm{P}$ and $\mathrm{B}$ which is not magnetized and the same as the $\mathrm{A}$ in dimensions shall be placed at the position which is symmetrical with the permanent magnet $\mathrm{A}$. The electrostatic induction forces of A and B to P shall be identical in order to eliminate the electrostatic induction. It is hard to adjust $\mathrm{P}$ to the middle position of $\mathrm{A}$ and $\mathrm{B}$ during the experiment, for example, the field strength to be measured shall be smaller than the actual value inevitably when the repulsion to $\mathrm{P}$ is reduced because the electrostatic attraction of $\mathrm{A}$ to $\mathrm{P}$ is greater than that of $\mathrm{B}$ if $\mathrm{P}$ is close to $\mathrm{A}$, the negative electric field exists around $\mathrm{A}$ and $\mathrm{P}$ has the negative charge; at this moment, the attraction to $\mathrm{P}$ shall be increased inevitably if $\mathrm{P}$ is charged positively, and the additional electric field for returning $\mathrm{P}$ to the position not stressed shall be increased inevitably; therefore, the field strength of $\mathrm{A}$ to be measured shall be greater than the actual value inevitably. During the experiment, the average value of the values of the great field strength and the small field strength measured shall be taken as the real field strength. The electric field generated by R and L shown in the figure shall be applied to offsetting the electric field generated by $\mathrm{A}$ in order to position $\mathrm{P}$ at the balanced position not stressed; and the field intensities of $\mathrm{R}$ and $\mathrm{L}$ at the place $\mathrm{P}$ shall be field intensities to be measured. Moreover, the results measured are $E_{1} \approx-242 \mathrm{~V} / \mathrm{m}, E_{2} \approx-133 \mathrm{~V} / \mathrm{m}$, $E_{3} \approx+196 \mathrm{~V} / \mathrm{m}, E_{4} \approx+97 \mathrm{~V} / \mathrm{m}, E_{5}=0$ and $E_{6}=0$ after many times of experiments.

$\varepsilon_{1}, \varepsilon_{2}, \varepsilon_{3}, \varepsilon_{4}, \varepsilon_{5}$ and $\varepsilon_{6}$ shall indicate the above-mentioned values calculated theoretically, respectively; the average velocity $v$ of the electron of magnetic domain of the permanent magnet must be confirmed before the theoretical calculation, and $\sigma=I / 2 a v$ and $n=\sqrt{1-V^{2} / C^{2}}$ can be confirmed when $v$ is confirmed. $\varepsilon_{1}=-224 \mathrm{~V} / \mathrm{m}$ can be acquired according to the formula $E_{1}=\left(E_{A 1}^{-}-E_{C 1}^{-}\right)-\left(E_{A}^{+}-E_{C 1}^{+}\right)$when $v=3.2 \times 10^{3} \mathrm{~m} / \mathrm{s}$ is confirmed after repeated calculation; $\varepsilon_{2}=-143 \mathrm{~V} / \mathrm{m}$ can be acquired in accordance with the formula $E_{2}$; and $\varepsilon_{3}=+514 \mathrm{~V} / \mathrm{m}, \varepsilon_{4}=+230 \mathrm{~V} / \mathrm{m}, \varepsilon_{5}=-268 \mathrm{~V} / \mathrm{m}$ and $\varepsilon_{6}=-242 \mathrm{~V} / \mathrm{m}$ can be acquired in accordance with the formula $E_{3}$.

Besides, $7.5 \%$ of errors exist between $\varepsilon_{1}$ and $\varepsilon_{2}$ and between $E_{1}$ and $E_{2}$, and more than $130 \%$ of errors exist between $\varepsilon_{3}$ and $\varepsilon_{4}$ and between $E_{3}$ and $E_{4}$, seen from the comparison between the theoretical result and the result measured; therefore, the theory is not in line with the actual condition, that is, the theory is incorrect due to $\varepsilon_{5}=-268 \mathrm{~V} / \mathrm{m}, E_{5}=0, \varepsilon_{6}=-242 \mathrm{~V} / \mathrm{m}$ and $E_{6}=0$.

Seen from the above-mentioned comparison, the previous theoretical analysis is not in line with the experimental result basically. However, the conclusion that both negative electric field and the positive electric field exist around the permanent magnet according to the theoretical analysis is in line with the actual condition. Well, why does such difference exist between the theoretical analysis and the actual result? To take one turn of rectangle current-carrying coil as the analysis model of the permanent magnet may be improper according to the analysis; therefore, the electron of the current-carrying coil moves straightly, while the molecular current of the magnetic domain of the permanent magnet revolves. Apparently, the moving electric fields formed as a result of two different motions shall be different from each other obviously.

We regard the permanent magnet as the one composed of the magnetic domains completely, and all molecular magnetic moments in all magnetic domains shall be the same. 


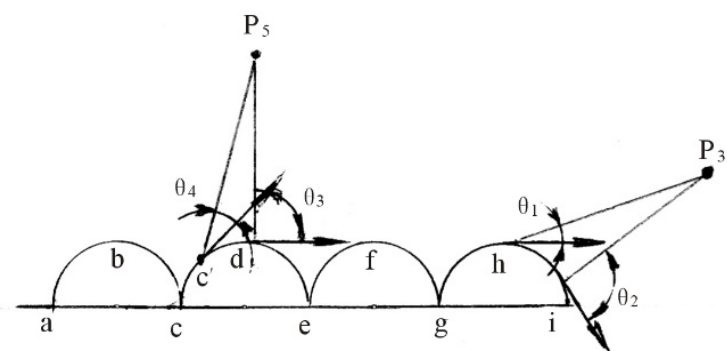

(a)

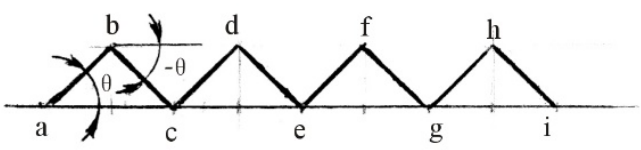

(b)

Figure 21. Actual current replaced by sawtooth current

In the micro view, the surface molecules on side of the permanent magnet like the cobblestones on the bench, and all electrons move along the surface of the cobblestone. In order to facilitate explanation, suppose the molecular currents of four magnetic moments in identical direction are sequenced, as shown in Figure 21 (a), apparently the directions of motion of the electrons at four points $\mathrm{b}, \mathrm{d}, \mathrm{f}$ and $\mathrm{h}$ are level only, that is, the directions of motion of the electrons of this four points are similar to the straight current of the rectangle coil only; while the directions of motion of the electrons at other points are not level. Taking the point $P_{5}$ in the figure (corresponding to the point $P_{5}$ of $E_{A 5}^{-}$in the experiment 5) as an example, the included angle $\theta_{3}$ shall be formed by the direction of motion of the electron and the direction of field strength of the electron when the electron moves horizontally, that is, the electron moves at the point $\mathrm{d}$ as shown in the figure, wherein $\theta_{3}$ is close to $90^{\circ} ; E_{5}$ to be acquired shall be the great negative field strength because it is mentioned before that the greater $\theta_{3}$ is, the greater the moving field strength would be. However, the direction of motion of the electron is changing time after time; the moving field strength shall be small if the included angle $\theta_{3}$ formed by the direction of motion of the electron and the direction of the field strength at point $\mathrm{C}$ is small and would be smaller than the positive electric field of corresponding proton; at this moment, $E_{5}$ shall be the positive electric field rather than the negative electric field. The moving trace of the whole electron may lead to $E_{5}=0$ naturally; as for the point $P_{3}$ shown in the figure corresponding to $E_{A 3}^{-}, E_{A 3}^{-}$measured actually shall be greater than the one calculated theoretically because $\theta_{1}$ analyzed theoretically is very small, while the actual $\theta$, such as $\theta_{2}$, may be very great; that is why the great difference exists between $E_{3}$ measured and the theoretical $\varepsilon_{3}$.

The deducing result which is in line with the actual condition can be acquired by analyzing the total field strength of the permanent magnet in presence of actual molecular current according to the above-mentioned analysis if the analysis model of the rectangle current coil is not used. However, we cannot understand the distribution condition of hundreds of millions molecular currents exactly, and analysis and calculation cannot be implemented easily although we understand the distribution condition. How to analyze the moving characteristics of the molecular currents and find the analysis model which is relatively in line with the actual condition after screening? We are not required to take the average automatic revolving velocity of the electron because the feeling strength of the permanent magnet shall not affect the distribution rule of the magnetic field and the synthetic electric field, and we should consider the changing rule of the above-mentioned angle $\theta$ mainly. We cannot acquire the whole changing rule of the angle $\theta$ starting from analysis of one molecular current because not every molecular magnetic moment is not in the same direction. Further, the synthetic electric field cannot be determined by the molecular current on the surface completely; however, the average ascending angle using the angle $\beta$ to indicate the motion of electron can be found under the condition that suppose that the total field strength is determined by the moving electrons on the topmost surface in terms of general changing effect in the direction of motion of the electron regardless of the distribution rule of the molecular magnetic moment, wherein $-\beta$ indicates the average descending angle, therefore such an angle $\beta$ shall be consistent with the general changing effect of the direction of motion of the electron. The motion of electron of this angle $\beta$ is shown in the Figure 21 (b), and the specific value of $\beta$ shall be determined by the experimental result naturally. Just like determination of current of the lead, the speeds of all free electrons forming the currents in the lead are not identical with each other completely, as well as the directions of motion of the free electrons. The current 
determined by the experiment shall be average value of the currents of all free electrons. Moreover, $\beta$ indicates the average value of the direction of motion of the electrons of all molecular currents.

Therefore, we set up the new analysis model of synthetic electric field of the permanent magnet. Different from the analysis model in 4.3.2, the electrons in this model shall move along with the sawtooth polygonal line shown in Figure 21(b) rather than move straightly along with the direction of current. In order to facilitate explanation, we call this new analysis model as the sawtooth current model; and as for the proton, the proton shall also be taken as static naturally in this current model. Therefore, all positive field intensities deduced before shall be kept still.

\subsubsection{Correction of Sawtooth Current Model to Total Field Strength}

Suppose the length of $a c$ is $d x, a b+b c$ is $d x^{\prime}$ when analyzing one polygonal line unit abc shown in the Figure 21 (b), $d x^{\prime}=d x / \cos \beta$ can be acquired. The distance of macro motion of the electron within the unit time shall not be changed if the current I is fixed; time required for the motion $a \rightarrow b \rightarrow c$ along with the polygonal line shall also be $\mathrm{dt}$ if the time required by the electron to move to $\mathrm{c}$ from a straightly is $\mathrm{dt}$; and the speed along with the polygonal line shall be $v^{\prime}=d x^{\prime} / d t=v / \cos \theta$ if the speed of the linear motion is $v=d x / d t$. The density of the surface charge of the electrons during motion along the polygonal line shall be $\sigma_{-}^{\prime}=-I / 2 a v^{\prime}=\sigma_{-} \cos \theta$; in order to simplify writing, order $f(\theta)=\left(1-v^{\prime 2} / C^{2}\right) /\left(1-\sin 2 \theta v^{\prime 2} / C^{2}\right)^{3 / 2} \cdot f\left(\theta_{1}\right)$ indicates $f(\theta)$ of the section ab with length of $d x^{\prime} / 2$, and $f\left(\theta_{2}\right)$ indicates $f(\theta)$ of the section bc with length of $d x^{\prime} / 2$ [Notes: $d x^{\prime}$ here is the scalar quantity and indicates the length only; the reason for this provisions is to simplify deduction] in order to distinguish because the angle of inclination of the polygonal line ab is $\beta$, while the angle of inclination of the section bc is $-\beta$. Moreover, $d E_{A i}^{-}$shown in Figure 17 of 4.3.2.1 shall be composed of the section ab and the section bc in presence of these provisions, that is,

$$
\begin{aligned}
& d E_{A \mathrm{i}}^{-}=\frac{k \sigma_{-}^{\prime} y d y}{r^{3}} \frac{1}{2} d x^{\prime} f\left(\theta_{1}\right)+\frac{k \sigma_{-}^{\prime} y d y}{r^{3}} \frac{\mathbf{1}}{\mathbf{2}} d x^{\prime} f\left(\theta_{2}\right) \\
& d E_{A \mathrm{i}}^{-}=\frac{k \sigma_{-}^{\prime} y d y d x}{r^{3}} \frac{\mathbf{1}}{\mathbf{2}}\left[f\left(\theta_{1}\right)+f\left(\theta_{2}\right)\right]
\end{aligned}
$$

shall be available after substituting $\sigma_{-}^{\prime}=\sigma_{-} \cos \theta$ and $d x^{\prime}=d x / \cos \theta$ to the formula above.

It is inevitable that $k \sigma_{-}^{\prime} y d y d x / r^{3}$ in the first half of the formula shall be identical with the first half during linear motion because the electric quantity of electron of straight line is the same as that of the surface current elements $d x$ and $d y$ or $d x^{\prime}$ and $d y$, and the static field intensities generated are partially the same naturally.

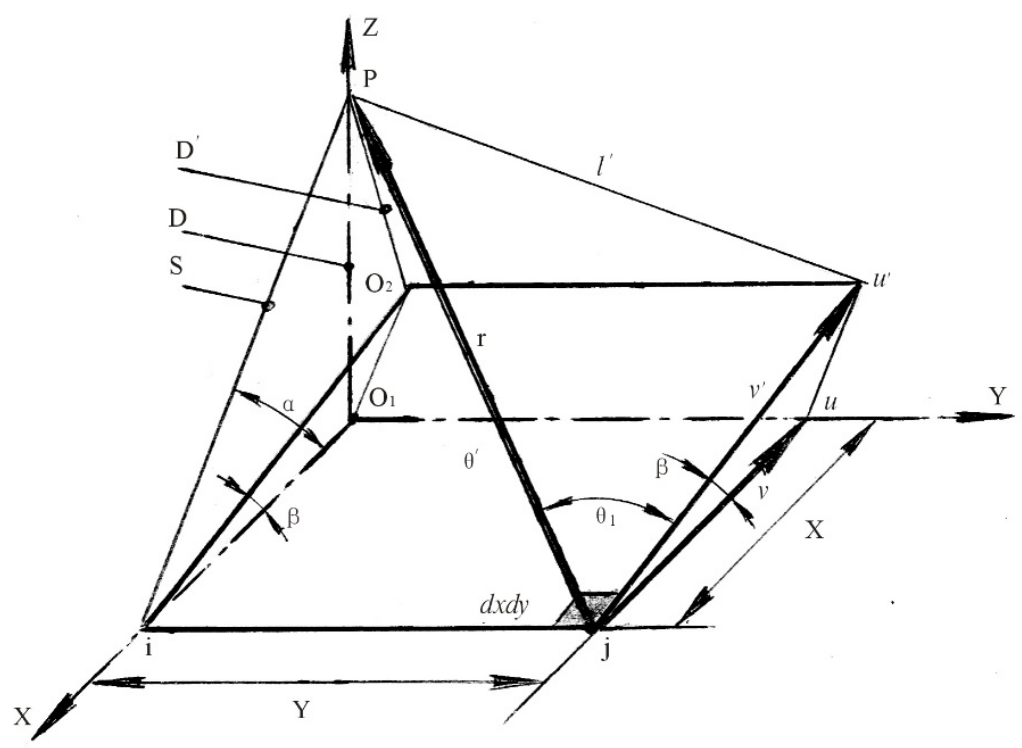

Figure 22. Relation analysis of $\sin \theta$ and $\beta$ 
$f\left(\theta_{1}\right)$ shall be analyzed first, mainly including the relation between $\theta_{1}$ and $\beta$. The ijuo plane system shown in Figure 22 has the common plane A with the Figure 17 in 4.3.21, wherein the point $\mathrm{j}$ indicates the position for analyzing the surface current element of $d E_{A 1}^{-}$in 4.3.2.1; the included angle $\beta$ is formed by the extended surface $i j u^{\prime} o_{2}$ and the extended surface $i j u o_{1}$ of the surface current element $d y d x^{\prime} / 2$ of the section $\alpha \mathrm{b}$ in the Formula (23), wherein in the figure, $\mathrm{D}^{\prime}$ indicates $\mathrm{Po}_{2}$ and is vertical to the plane ${ }^{2} \mathrm{ju}^{\prime} \mathrm{O}_{2} ; \Delta \mathrm{Po}_{2} u^{\prime}$ is also vertical to the plane $i j u^{\prime} O_{2}$ naturally. Therefore, the $\angle p u^{\prime} j$ is the right angle inevitably; the angle $\theta_{1}$ shown in the figure is the angle we want because $j u^{\prime}$ is the direction of the moving velocity $v^{\prime}$ of the electron of the polygonal line, $\sin \theta_{1}=l^{\prime} / r, \quad\left(l^{\prime}=p u^{\prime}\right)$. The four points $\mathrm{i}, o_{1}, o_{2}$ and $\mathrm{p}$ are positioned in one plane, order ip=S, $S=\sqrt{x^{2}+D^{2}}$ and

$D^{\prime}=S \sin (\alpha-\beta)=S(\sin \alpha \cos \beta-\cos \alpha \sin \beta)$ can be acquired. Moreover, the formula $D^{\prime}=D \cos \beta-x \sin \beta$ can be acquired after substituting $\sin \alpha=D / S$ and $\cos \alpha=x / S$ into the formula $D^{\prime}$. From the right angle $\Delta p o_{2} u^{\prime}, \quad l^{\prime}=\sqrt{y^{2}+D^{\prime 2}}=\sqrt{y^{2}+(D \cos \beta-x \sin \beta)^{2}}$ can be acquired; further, the Formula

$$
\sin \theta_{1}=\frac{1}{r} \sqrt{y^{2}+(D \cos \beta-x \sin \beta)^{2}}
$$

can be available when substituting $l^{\prime}=\sqrt{y^{2}+D^{\prime 2}}=\sqrt{y^{2}+(D \cos \beta-x \sin \beta)^{2}}$ to the formula $\sin \theta_{1}$.

The $f\left(\theta_{2}\right)$ shall be analyzed by using the identical analysis method, and the formula

$$
\sin \theta_{2}=\frac{1}{r} \sqrt{y^{2}+(D \cos \beta+x \sin \beta)^{2}}
$$

can be acquired easily after changing the angle $\beta$ as $-\beta$.

$$
E_{A 1}^{-}=\frac{k \sigma_{-}^{\prime}}{A}\left\{\ln \frac{y^{2}+J^{2} / B^{2}}{\left(M_{1}+\sqrt{y^{2}+\frac{J^{2}}{B^{2}}+M_{1}^{2}}\right)\left(M_{2}+\sqrt{\left.y^{2}+\frac{J^{2}}{B^{2}}+M_{1}^{2}\right)}\right.}\right\}_{y 1}^{y 2}
$$

can be acquired by using the integration solving method after substituting $\sin \theta_{1}$ and $\sin \theta_{2}$ into the Formula

In the Formula (26)

$$
\begin{array}{lll}
A=1-\sin ^{2} \beta v^{\prime 2} / c^{2} & B=\frac{1-v^{\prime 2} / c^{2}}{A^{2}} & G=\frac{\sin 2 \beta v^{\prime 2} / c^{2}}{2 A^{2}} \\
M_{1}=\frac{a+D G}{B} & M_{2}=\frac{a-D G}{B} & J^{2}=\frac{D^{2}\left(1-\cos ^{2} \beta v^{\prime 2} / c^{2}\right)}{A^{2}}-D^{2} G^{2}
\end{array}
$$

$E_{C 1}^{-} \quad$ can be acquired after changing D in the Formula (26) as $D+2 a$; and the formula

$$
E_{A_{3}^{\prime}}^{-}=\frac{k \sigma_{-}^{\prime} n^{\prime}}{A^{2}}\left\{\ln \frac{\sqrt{y^{\prime 2}+J^{2}}}{\frac{a n^{\prime}}{A}+\sqrt{y^{\prime 2}+J^{2}+\frac{a^{2} n^{\prime 2}}{A^{2}}}}\right\}_{y_{1}^{\prime}}^{y_{2}^{\prime}}+\frac{k \sigma_{-}^{\prime} n^{\prime}}{A^{2}}\left\{\ln \frac{\sqrt{y^{\prime \prime 2}+J^{2}}}{\frac{a n^{\prime}}{A}+\sqrt{y^{\prime \prime 2}+J^{2}+\frac{a^{2} n^{\prime 2}}{A^{2}}}}\right\}_{y_{1}^{\prime \prime}}^{y_{2}^{\prime \prime}}
$$

Can be acquired by using the deducing method which is similar to that of $E_{A^{2}}^{-}$, specifically changing $h=5 \times 10^{-3} \mathrm{~m}$ as $h=10^{-2} \mathrm{~m}$, wherein in the formula, $n^{\prime}=\sqrt{1-v^{\prime 2} / C^{2}}, y^{\prime}=y+D G, y^{\prime \prime}=y-D G$, and A, $\mathrm{J}$ and $\mathrm{G}$ are the same as those in the Formula (26).

$E_{C 3}^{-}$can be acquired after changing $\mathrm{D}$ in the Formula (27) as $D+2 a$; and the formula

$$
E_{B 3^{\prime}}^{-}=k \sigma_{-}^{\prime}\left[\sin ^{-1} \sqrt{\frac{(a+H)^{2}}{(a+H)^{2}+S^{2}}} \sin \operatorname{tg}^{-1} \frac{n^{\prime} z}{S A}+\sin ^{-1} \sqrt{\frac{(a-H)^{2}}{(a-H)^{2}+S^{2}}} \sin \operatorname{tg}^{-1} \frac{n^{\prime} z}{S A}\right]_{D}^{D+2 a}
$$


can be acquired by using the deducing method which is similar to that of $E_{A^{4}}^{-}$, specifically changing $h=5 \times 10^{-3} \mathrm{~m}$ as $h=10^{-2} \mathrm{~m}$, wherein in the formula

$$
H=\frac{h \sin 2 \beta v^{\prime 2} / C^{2}}{2 A^{2}} \quad h^{\prime}=\sqrt{1-v^{\prime 2} / C^{2}} \quad S=\frac{h^{2}}{A^{2}}\left(1-\cos 2 \beta v^{\prime 2} / C^{2}\right)-H^{2}
$$

$E_{B^{\prime} 4}^{-}$can be acquired after changing $h=5 \times 10^{-3} \mathrm{~m}$ in the Formula (28) as $h=10^{-2} \mathrm{~m} ; E_{D^{\prime} 3}^{-}$can be acquired after changing $h$ in the Formula (28) as $h+2 a$; and $E_{D^{\prime} 4}^{-}$can be acquired after changing $h=5 \times 10^{-3} \mathrm{~m}$ of $E_{D^{\prime} 3}^{-}$ as $h=10^{-2} \mathrm{~m}$. Further, the formula

$$
E_{A S^{\prime}}^{-}=k \sigma_{-}^{\prime}\left[\sin ^{-1} \sqrt{\frac{(a+H)^{2}}{(a+H)^{2}+S^{2}}} \sin \operatorname{tg}^{-1} \frac{n^{\prime} z}{S A}+\sin ^{-1} \sqrt{\frac{(a-H)^{2}}{(a-H)^{2}+S^{2}}} \sin \operatorname{tg}^{-1} \frac{n^{\prime} z}{S A}\right]_{-a}^{a}
$$

can be acquired by using the similar deduction, wherein in the Formula (29), $E_{A 6^{\prime}}^{-}$can be acquired by changing $h=5 \times 10^{-3} \mathrm{~m}$ as $h=10^{-2} \mathrm{~m}$.

The value $\beta$ at the angles $45^{\circ}, 20^{\circ}, 39^{\circ}, 30^{\circ}, 36^{\circ}, 35^{\circ}$ and $35.2^{\circ}$ are deduced according to the formula above. $\varepsilon_{1}^{\prime}=-238.73 \mathrm{~V} / \mathrm{m}, \varepsilon_{2}^{\prime}=-134.76 \mathrm{~V} / \mathrm{m}, \varepsilon_{3}^{\prime}=+186.9 \mathrm{~V} / \mathrm{m}, \varepsilon_{4}^{\prime}=+106 \mathrm{~V} / \mathrm{m}, \varepsilon_{5}^{\prime}=-0.926 \mathrm{~V} / \mathrm{m}$ and $\varepsilon_{6}^{\prime}=-0.59 \mathrm{~V} / \mathrm{m}$ can be acquired when $\beta=35.2^{\circ}$. Except $9.4 \%$ of error of $\varepsilon_{4}^{\prime}$ and $E_{4}$, the other errors are less than $5 \%$ after comparing the result measured $E_{1}=-242 \mathrm{~V} / \mathrm{m}$ with $E_{2}=-133 \mathrm{~V} / \mathrm{m}, E_{3}=+196 \mathrm{~V} / \mathrm{m}, E_{4}=+97 \mathrm{~V} / \mathrm{m}$ and $E_{1}=E_{6}=0$; therefore, it can be considered the analysis method taking the sawtooth current as the analysis model is in line with the actual condition basically.

\subsection{Constant Light Speed is the Source of Magnet}

The Lorentz force phenomenon and the phenomenon that the static field existed around the permanent magnet are explained respectively, and the problem of interaction of the parallel wires with currents is clarified more scientifically and reasonably according to the basic concept of length contraction of the electric field in the direction of motion. Then we shall analyze the reason why the permanent magnet has the phenomenon that like poles repel but opposite poles attract.

In order to explain simply, two permanent magnets A and B which are the same shall be taken to explain; the revolving directions of the molecular currents of the magnetic domain $\mathrm{A}$ and the magnetic domain $\mathrm{B}$ are the same when the pole $\mathrm{N}$ of A faces the pole $\mathrm{S}$ of B. $Q_{A}^{-}$indicates the electric quantity of the negative electron moving in the magnetic domain $\mathrm{A}$, and $Q_{A}^{+}$indicates the electric quantity of the proton having the electric quantity identical with that of the $Q_{A}^{-}$in the magnetic domain A; similarly, $Q_{B}^{-}$and $Q_{B}^{+}$indicates the electric quantities of the moving electron and the static proton in the magnetic domain $\mathrm{B} ; u$ indicates the revolving speeds of the moving electrons in the magnetic domains $\mathrm{A}$ and $\mathrm{B}$. In order to facilitate explanation, order $E_{x}^{+} Q_{x}^{+}=E_{x}^{-} Q_{x}^{-}=E Q$ indicates repulsion, and $E_{x}^{-} Q_{x}^{+}=E_{x}^{+} Q_{x}^{-}=-E Q$ indicates attraction.

As for $Q_{A}^{+}$, the repulsion of the field strength $E_{B}^{+}$of $Q_{B}^{+}$to $Q_{A}^{+}$shall be $F_{A^{+}}^{B^{+}}=E_{B}^{+} Q_{A}^{+}=E Q$; while the field strength $E_{B}^{-}$of $Q_{B}^{-}$to $Q_{A}^{+}$shall be taken as attraction, and $E_{B}^{-}=E_{B}^{-} / \sqrt{1-u^{2} / C^{2}}=E_{B}^{-}\left(1+u^{2} / 2 C^{2}\right)$ shall be available (see 4.2 for proof) because $E_{B}^{-}$moves opposite to $Q_{A}^{+}$at the speed $u$ and the electric field in motion shall be contracted and increased; moreover, if the attraction of $Q_{B}^{-}$to $Q_{A}^{+}$shall be $F_{A^{+}}^{B^{-}}=E_{B}^{-} Q_{A}^{+}=-E Q\left(1+u^{2} / 2 C^{2}\right)$, the total force to $Q_{A}^{+}$shall be

$$
F_{A^{+}}=F_{A+}^{B+}+F_{A+}^{B-}=E Q\left[1-\left(1+u^{2} / 2 C^{2}\right)\right]=-E Q u^{2} / 2 C^{2} .
$$

As for $Q_{A}^{-}$, the field strength $E_{B}^{+}$of $Q_{B}^{+}$shall move opposite to $Q_{A}^{-}$at the speed of $-u$, and $E_{B}^{+}$shall be increased as $E_{B}^{+}=E_{B}^{+}\left(1+u^{2} / 2 C^{2}\right)$; the attraction of $E_{B}^{t^{+}}$to $Q_{A}^{-}$shall be $F_{A^{-}}^{B^{+}}=E_{B}^{+} Q_{A}^{-}=-E Q\left(1+u^{2} / 2 C^{2}\right)$, and the field strength $E_{B}^{-}$of $Q_{B}^{-}$shall be static with respect to $Q_{B}^{-}$, and the repulsion of the two shall be $F_{A^{-}}^{B^{-}}=E_{B}^{-} Q_{A}^{-}=E Q$; the total force to $Q_{A}^{-}$shall be $F_{A-}=F_{A-}^{B+}+F_{A-}^{B-}=-E Q\left[\left(1+u^{2} / 2 C^{2}\right)-1\right]=-E Q u^{2} / 2 C^{2}$. Moreover, the attracting power of the two permanent magnets $\mathrm{A}$ and $\mathrm{B}$ shall be $F_{1}=F_{A^{+}}+F_{A^{-}}=-E Q u^{2} / \mathrm{C}^{2}$. 
Then turn to the condition of the pole $\mathrm{N}$ of A facing the pole $\mathrm{N}$ of $\mathrm{B}$; at this moment, the revolving directions of the molecular currents of the magnetic domains $\mathrm{A}$ and $\mathrm{B}$ are opposite, in which the field strength of $E_{B}^{-}$to $Q_{A}^{-}$ shall be increased to $E_{B}^{-}=E_{B}^{-}\left[1+(2 u)^{2} / 2 C^{2}\right]=E_{B}^{-}\left(1+2 u^{2} / C^{2}\right)$ if the moving speed of the field strength $E_{B}^{-}$of $Q_{B}^{-}$shall be $2 u$ with respect to $Q_{A}^{-}$, and the acting force of $E_{B}^{-}$to $Q_{A}^{-}$shall be $F_{A-}^{B-}=E_{B}^{-} Q_{A}^{-}=E Q\left(1+2 u^{2} / C^{2}\right)$; and the field strength of $E_{B}^{+}$to $Q_{A}^{-}$shall be increased to $E_{B}^{+^{\prime}}=E_{B}^{+}\left(1+u^{2} / 2 C^{2}\right)$ if the field strength $E_{B}^{+}$of $Q_{B}^{+}$shall move with respect to $Q_{A}^{-}$at the speed $u$, and the acting force of $E_{B}^{+^{\prime}}$ to $Q_{A}^{-}$shall be $F_{A-}^{B+}=E_{B}^{+} Q_{A}^{-}=-E Q\left(1+u^{2} / 2 C^{2}\right) ; Q_{B}^{+}$shall be static with respect to $Q_{A}^{+}$, and the field strength $E_{B}^{+}$of $Q_{B}^{+}$to $Q_{A}^{+}$shall be $F_{A+}^{B+}=E_{B}^{+} Q_{A}^{+}=E Q$; the acting force of $E_{B}^{-}$to $Q_{A}^{+}$shall be $F_{A+}^{B-}=E_{B}^{-} Q_{A}^{+}\left(1+u^{2} / 2 C^{2}\right)$ if the field strength $E_{B}^{-}$of $Q_{B}^{-}$moves with respect to $Q_{A}^{+}$at the speed $u$. Moreover, the total repulsion of $\mathrm{A}$ and $\mathrm{B}$ shall be

$$
F_{2}=F_{A-}^{B-}+F_{A-}^{B+}+F_{A+}^{B+}+F_{A+}^{B-}=E Q\left[\left(1+2 u^{2} / C^{2}\right)+1-2\left(1+u^{2} / 2 C^{2}\right)\right]=E Q u^{2} / C^{2} .
$$

The interaction force $F_{1}=-E Q u^{2} / C^{2}$ of the two shall be considered as the attraction which indicates the opposite poles attract each other according to the above-mentioned analysis when the pole $\mathrm{N}$ of A faces the pole $\mathrm{S}$ of $\mathrm{B}$; and the interaction force $F_{2}=E Q u^{2} / C^{2}$ of the two shall be considered as the repulsion which indicates the like poles repel when the pole $\mathrm{N}$ of $\mathrm{A}$ faces the pole $\mathrm{S}$ of $\mathrm{B}$. moreover, the attraction shall be equal to the repulsion.

Why does the permanent magnet attract the iron-containing substances? This is because the iron-containing substances contain a plenty of magnetic domains, and the heteropole of the magnetic domain in the iron-containing substance will be attracted by the pole $\mathrm{N}$ or $\mathrm{S}$ of the permanent magnet when the magnetic domain is close to the permanent magnet, while the homopole shall be repelled by the pole $\mathrm{N}$ or $\mathrm{S}$ of the permanent magnet. This attraction and repulsion shall generate the couples to the magnetic domain; therefore the magnetic domain shall revolve, and further the heterpole shall be attracted after approaching the permanent magnet.

All magnetic phenomena (electro-magnetic induction, wherein the electromagnetic wave shall be clarified below otherwise) we see in daily lives are from the length contractions of the electric fields in the direction of motion according to the above-mentioned analysis, that is, the contractions of the electric fields in the direction of motion is taken as the source of magnet. Why do the electric fields contract in the direction of motion? As everyone knows, the contraction of length in the direction of motion is taken as the scientific verification of special relativity and Lorentz transformation, and also the objective rule which has been proved in practices. While the Lorentz transformation is on the basis of the rule of constant light speed (Law of Constant Light Speed) existing in the nature. Therefore, the real source of all magnetic phenomena is the natural rule of constant light speed

We can image that no contraction of the electric field shall occur in the direction of motion if the rule of constant light speed does not exist in the nature, no magnet shall exist if no natural contraction of the electric field exists, and further, all modern and scientific achievements, such as the motor, the computer, the remote control, etc. shall not exist if there is no magnet. Therefore, to sum up, the law of constant light speed is the key that is granted to human body by God to open the door of the modernization.

\section{Variable Motion of Electric Field}

\subsection{Generation of Electric Wave}

A section of "infinite" plane conductor is shown in Figure 22 (a), static positive electric field generated by the positive electric field of equivalent electric quantity with the negative electron in conductor; the dotted line indicates its power line; $\boldsymbol{E}_{\boldsymbol{o}}$ indicates its electric field intensity. All the negative electrons in the conductor are in the same variable motion. It is indicated in Formula 3.7 that, when the electron is in variable motion, it will be propagated at the velocity of light in its generated electric field; propagation velocity: $C=d x / d t$. Given that the electron is moving along direction of $\mathrm{Y}$ coordinate according to the law of motion $y=f(t)$, the electron will be propagated at the velocity of light $\mathrm{C}$ in its generated electric field, resulting in the distortion of electric field. Line E indicates the electric field of such electrons (as shown in Figure 22 (a)). As a result of electron motion, the electron will be propagated, resulting in the distortion of power line. Given that the law of motion at Point $\mathrm{P}$ on 
the power line $E$ is set as: $y=f(t)$, then the law of motion at Point $P^{\prime}$ at Section $l$ behind Point P must be set as: $y=f(t-l / c)$. The movement velocity of electric field at Point $\mathrm{P}$ along the direction in $\mathrm{Y}$ coordinate shall be:

$$
u=\frac{d y}{d t}=\frac{d f(t)}{d t}
$$

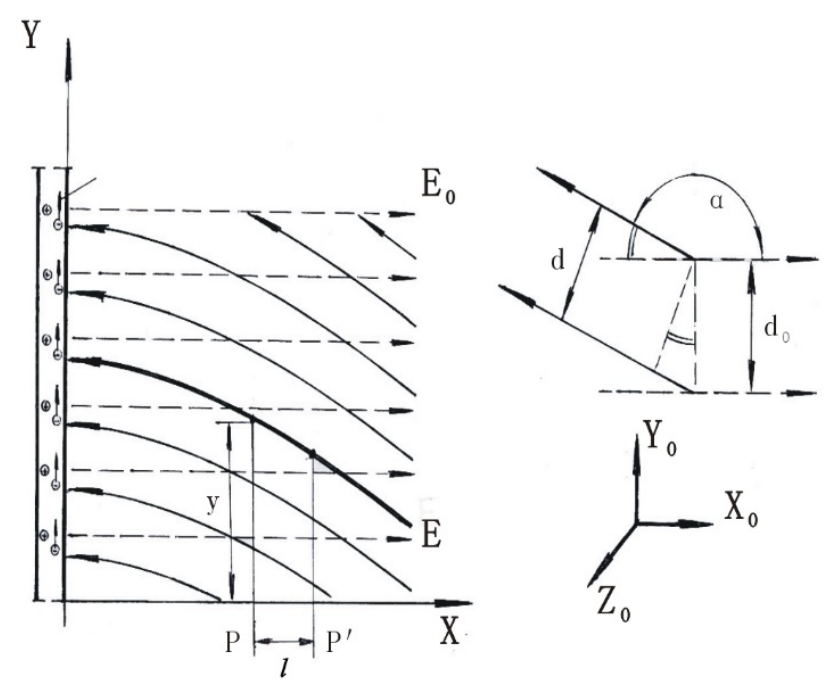

(a)

(b)

Figure 22. Distortion of power line caused by the variable motion of negative electrons

It can be seen from Figure (a) that, because of tilt, the distance between the lines $\boldsymbol{E}$ will become smaller. The relationship between the distance $d$ between the tilted lines $\boldsymbol{E}$ and the distance $d_{0}$ between the electric field lines $\boldsymbol{E}_{0}$ of positive electron shall be shown in Figure 22(b). As shown in Figure 22(b), if the included angle between $\boldsymbol{E}$ and $\boldsymbol{E}_{0}$ is set as $\alpha$, then $d=d_{0}|\cos \alpha|$. Since $\cos \alpha$ represents a negative value, so $d=d_{0}(-\cos \alpha)$. Since the electric field intensity is inversely proportional to the distance between the power lines, so the ratio of scalars between $\boldsymbol{E}$ and $\boldsymbol{E}_{0}$ shall be as follows:

$$
\frac{E}{E_{0}}=\frac{d_{0}}{d}=\frac{1}{-\cos \alpha}
$$

Namely, $\mathrm{E}=-\mathrm{E}_{0} / \cos \alpha . \boldsymbol{E}$ can be decomposed into two components: One component is parallel to $x$, which is expressed by $\boldsymbol{E}_{x}$; one component is perpendicular to $x$, expressed by $\boldsymbol{E}_{y}$. Obviously:

$$
\boldsymbol{E}_{x}=\boldsymbol{E} \cos \alpha=\frac{-E_{0}}{\cos \alpha} \cdot \cos \alpha \boldsymbol{X}_{0}=-E_{0} \boldsymbol{X}_{0}
$$

$$
\text { ( } \boldsymbol{X}_{0} \text {-represents the unit vector along the direction of } x \text { coordinate) }
$$

Since $\boldsymbol{E}_{x}$ belongs to a moving electric field, its velocity of movement along the direction of y coordinate shall be expressed as $\boldsymbol{u}$; the electric field is a kind of matter; according to the Special Theory of Relativity, the matter (velocity of movement: $\boldsymbol{u}$ ) will be contracted in the direction of motion according to the proportion $\sqrt{1-u^{2} / c^{2}}$; therefore, $\boldsymbol{E}_{x}$ will be contracted in the direction of y coordinate according to the proportion $\sqrt{1-u^{2} / c^{2}}$, so that the distance between the power lines will naturally become smaller; the electric field intensity shall be inversely proportional to the distance between the power lines; as a result of contraction of electric field, $\boldsymbol{E}_{x}$ can be converted into: 


$$
\boldsymbol{E}_{x}^{\prime}=\frac{\boldsymbol{E}_{x}}{\sqrt{1-u^{2} / c^{2}}}=-\frac{E_{o}}{\sqrt{1-u^{2} / c^{2}}} \boldsymbol{X}_{o}
$$

Since there is no motion of $\boldsymbol{E}_{y}$ in the direction of coordinate (namely, the negative electron has not moved in the conductor along the direction of $x$ coordinate), so the electric field has not been contracted in the direction of $x$ coordinate. There is no any relationship between $\boldsymbol{E}_{y}$ and $\boldsymbol{u}$, thus:

$$
\boldsymbol{E}_{y}^{\prime}=\boldsymbol{E}_{y}=\boldsymbol{E} \sin \alpha=\frac{-E_{o}}{\cos \alpha} \cdot \sin \alpha \boldsymbol{Y}_{o}=-E_{o} \operatorname{tg} \alpha \boldsymbol{Y}_{o}
$$

In the above formula, since $\operatorname{tg} \alpha$ represents a negative value, so $\boldsymbol{E}_{y}^{\prime}$ has the same direction as $\mathbf{Y}_{\mathrm{o}}$.

$$
\boldsymbol{E}^{\prime}=\boldsymbol{E}_{x}^{\prime}+\boldsymbol{E}_{y}^{\prime}=\frac{-E_{o}}{\sqrt{1-u^{2} / c^{2}}} \cdot \boldsymbol{X}_{o}-E_{o} \operatorname{tg} \alpha \boldsymbol{Y}_{o}
$$

As can be seen from Figure 22 (a), $\operatorname{tg} \alpha=d y / d x$. Since $u=d y / d t, c=d x / d t$, therefore:

$$
\operatorname{tg} \alpha=\frac{d y}{d t} \cdot \frac{d t}{d x}=\frac{u}{C}
$$

Since the movement velocity of electric field of electron along the direction of $\mathrm{Y}$ coordinate is expressed as $u=d y / d t$, according to Section 3.1, the moving electric field shall be considered as the magnetic field. According to the above Formula (1), the following formula can be derived:

$$
\boldsymbol{B}=\frac{1}{C^{2}} \cdot \boldsymbol{u} \times \boldsymbol{E}=\mu_{0} \varepsilon_{0} \boldsymbol{u} \times \boldsymbol{E}
$$

Since $u$ is much less than $C$ according to the Formula (35), $\operatorname{tg} \alpha$ shall be minimal, namely, $\alpha$ shall be minimal, so it can be considered that $\boldsymbol{u} \perp \boldsymbol{E}$. The Formula (35) can be written as the scalar formula below:

$$
B=\mu_{0} \varepsilon_{0} u \times E
$$

From the above analysis, when the in-plane electron is in variable motion, it will be propagated at the velocity of light in its generated electric field, resulting in the distortion of electric field generated by the electron. As a result of distortion of electric field, there exists the electric field intensity $E_{y}=-E_{0} u / C$ in the direction of electron motion. As a result of movement velocity $u$ of electric field of electron, the electric field moving at velocity $u$ shall be considered as the magnetic field (magnetic induction intensity: $B=\mu_{0} \varepsilon_{0} u E$ ). Obviously, the electromagnetic wave refers to the process in which $E_{y}$ and $B$ are propagated in the distorted electric field.

\subsection{Energy Output of Electromagnetic Wave}

As previously mentioned, there still exist the respective electric fields of positive \& negative electrons in the conductor. The electric field has its electric field energy. Why does the electric field energy exist around the conductor when there is no current flowing in the conductor? Obviously, the reason is that the electric field energy of positive electron and the electric field energy of negative electron may be offset each other. It is indicated in Formula 5.1 that, when the electromagnetic wave is generated, $\boldsymbol{E}=\boldsymbol{E}_{0} / \cos \alpha,|E|>\left|E_{0}\right|$, the electric field energy of $\boldsymbol{E}$ is greater than the electric field energy of $\boldsymbol{E}_{0}$. After both are offset, there inevitably exists the residual electric field energy which is the energy output of electromagnetic wave. Considering that the actual existing electric field energy is basically the electric field energy of negative electron, therefore, the electric field energy of negative electron can be defined as the positive electric field energy; the electric field energy of positive electron can be defined as the negative electric field energy. Thus, the electric field energy per unit volume in electromagnetic wave shall be the difference between the moving electric field intensity of $E^{\prime}$ and the static electric field energy of $E_{0}$.

$$
W=\frac{\varepsilon_{o}}{2} \boldsymbol{E}^{\prime 2}-\frac{\varepsilon_{o}}{2} E_{o}^{2}=\frac{\varepsilon_{o}}{2}\left(\boldsymbol{E}_{x}^{\prime 2}+\boldsymbol{E}_{y}^{\prime 2}\right)-\frac{\varepsilon_{o}}{2} \boldsymbol{E}_{o}^{2}
$$

Since there is no any relationship between the size of electric field energy and the direction of E, the above formula can be written as the scalar formula. After the Formula (34) is substituted into the scalar formula, the following formula can be obtained:

$$
W=\frac{\varepsilon_{0} E_{0}^{2}}{2\left(1-u^{2} / c^{2}\right)}+\frac{\varepsilon_{o}}{2} E_{y}^{2}-\frac{\varepsilon_{0}}{2} E_{o}
$$


$1 /\left(1-u^{2} c^{2}\right)$ can be expanded as $1+u^{2} / c^{2}+u^{4} / c^{4}+$ according to the binomial progression series. Since $u$ is very small relative to $\mathrm{c}$, so $u^{4} / c^{4}$ and the subsequent items can be ignored. After $1 /\left(1-u^{2} c^{2}\right)=1+u^{2} / c^{2}$ is substituted into the above formula, the following formula can be obtained:

$$
W=\frac{\varepsilon_{o}}{2} E_{o}^{2}+\frac{\varepsilon_{o} u^{2} E_{0}^{2}}{2 c^{2}}+\frac{\varepsilon_{o}}{2} E_{y}^{2}-\frac{\varepsilon_{o}}{2} E_{o}^{2}=\frac{\varepsilon_{0}}{2} E_{y}^{2}+\frac{\varepsilon_{o} u^{2} E_{o}^{2}}{2 c^{2}}=W_{1}+W_{2}
$$

Where, $\mathrm{W}_{1}=\varepsilon_{o} E_{y}^{2} / 2$ represents the electric field energy per unit volume generated by the electromagnetic wave in the direction of y; $W_{2}=\varepsilon_{o} u^{2} E_{o}^{2} / 2$ represents the electric field energy per unit volume generated by the electromagnetic wave in the direction of $x$. As a result of motion contraction, the electric field energy per unit volume will be increased. What is the relationship between $W_{1}$ and $W_{2}$ ? Because of $E_{y}=E_{0} \operatorname{tg} \alpha, \operatorname{tg} \alpha=u / c$, $W_{2}$ can be transformed into:

$$
W_{2}=\frac{\varepsilon_{o}}{2} E_{o}^{2} \frac{u^{2}}{c^{2}}=\frac{\varepsilon_{o}}{2} E_{o}^{2} \operatorname{tg}^{2} \alpha=\frac{\varepsilon_{o}}{2} E_{y}^{2}=W_{1}
$$

It is indicated in the Formula (39) that, there is the same size of energy $W_{1}$ and $W_{2}$ propagated via the electromagnetic wave.

In the Formula (36), $\boldsymbol{B}=\mu_{0} \varepsilon_{0} \boldsymbol{u} \times \boldsymbol{E}$,i.e. $u E=B / \mu_{0} \varepsilon_{0}$. Because of $\mu_{0} \varepsilon_{0}=1 / c^{2}, B=\mu_{0} H$; therefore:

$$
W_{2}=\frac{\varepsilon_{o}}{2 C^{2}} E_{o}^{2} u^{2}=\frac{\varepsilon_{o}}{2}\left(\mu_{0} \varepsilon_{o}\right) \cdot \frac{B^{2}}{\mu_{0}^{2} \varepsilon_{0}^{2}}=\frac{B^{2}}{2 \mu_{0}}=\frac{\mu_{0}}{2} H^{2}
$$

It is indicated In the Formula (40) that, the increased energy caused by the contraction motion of electric field is the magnetic field energy. Therefore, it is indicated In the Formula (39) that, the energy propagated via the electromagnetic wave shall be of the same size as the magnetic field energy.

After the Formula (40) is substituted into the Formula (38), the following formula can be obtained:

$$
W=W_{1}+W_{2}=\frac{\varepsilon_{o}}{2} E_{Y}^{2}+\frac{\mu_{o}}{2} H^{2}
$$

Since $W_{1}=W_{2}$, the result can be derived from the Formula (41): $E_{y}=H \sqrt{\mu_{0} / \varepsilon_{0}}$, so that the Formula (41) can be transformed into:

$$
W=2 W_{1}=\varepsilon_{o} E_{y} \cdot E_{y}=\varepsilon_{o} E_{y} H \sqrt{\mu_{o} / \varepsilon_{o}}=E_{y} H \sqrt{\mu_{0} \varepsilon_{0}}
$$

Since $\sqrt{\mu_{o} \varepsilon_{o}}=1 / \mathrm{c}$, the above formula can be transformed into:

$$
W=\frac{E_{y} H}{c}
$$

W represents the energy per unit area propagated via the electromagnetic wave within one second.

$$
\text { Supposing that } \boldsymbol{S}=\boldsymbol{E}_{v} \times \boldsymbol{H}
$$
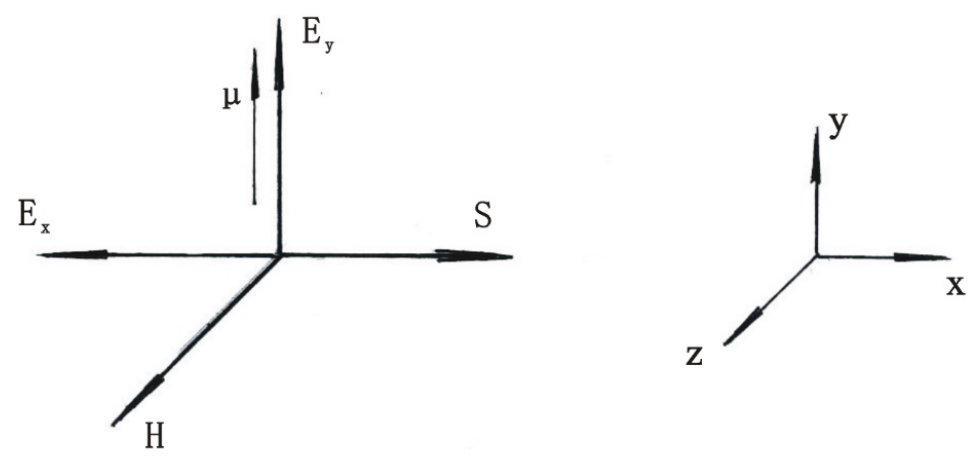

Figure 23. S. H.E $y$ vector diagram 
Since $\boldsymbol{H}=\boldsymbol{B} / \mu_{o}=\boldsymbol{u} / \mu_{o} c^{2} \times \boldsymbol{E}_{x}$, perpendicular to $\boldsymbol{E}_{x}$ and $\boldsymbol{u}$ in the direction of $\boldsymbol{Z}$ coordinate, as shown in Figure 23, $\boldsymbol{S}=\boldsymbol{E}_{y} \times \boldsymbol{H}$ in the direction of $x$ coordinate, i.e. in the direction of propagation of electromagnetic wave, so $S$ expresses the intensity of energy transferred toward its direction of propagation via the electromagnetic wave, namely, the Poynting vector defined in Electromagnetics.

\subsection{Analysis of Electromagnetic Wave}

From the above analysis of energy output and generation of electromagnetic wave, what is the difference between the electromagnetic wave and the electromagnetic wave introduced in Electromagnetics?

It is indicated in Electromagnetics that, the spatially-varying electric field will generate a varying magnetic field, and vice versa, the varying magnetic field will generate a varying electric field, which is called the electromagnetic wave to follow in a circle incessantly. The varying electric and magnetic fields are considered as the substances. I believe that, any substances are impossible to be produced, including the electric and gravitational fields (as described in Section 2.14, the magnetic field can not be a substance but an electric field caused by contraction motion). The varying electric field is also impossible to be generated naturally without foundation. As previously mentioned, the actually observed varying electric field refers to an effect caused by distortion of inherent electric field of electron in variable motion along with the electron from the near to the distant. Further descriptions are as follows:

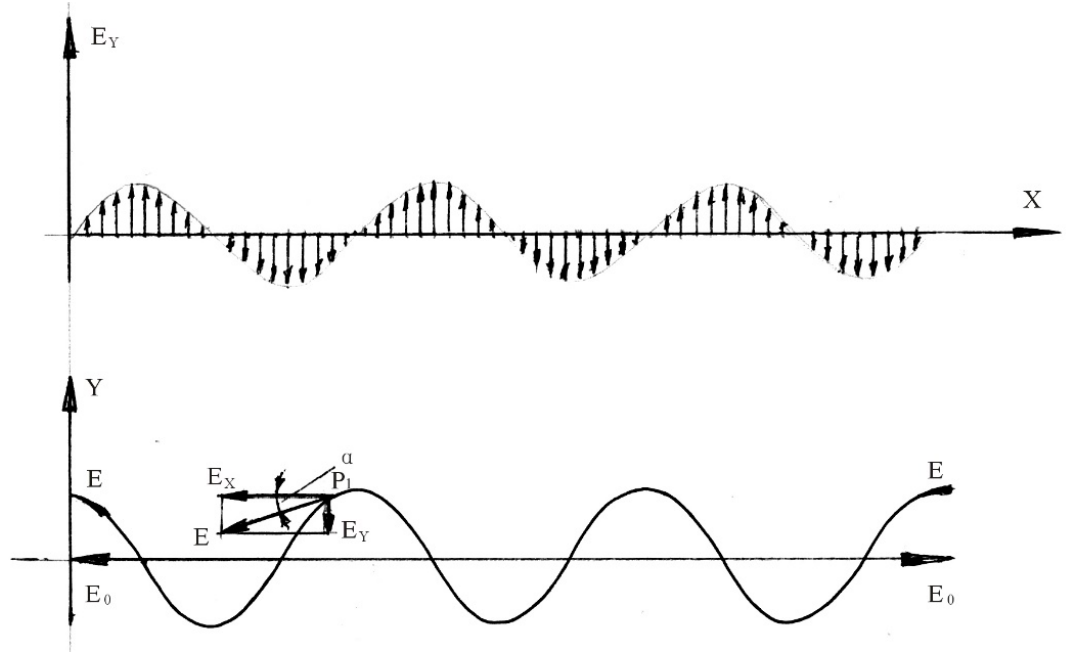

Figure 24. Description of the actual situation of electromagnetic wave

As shown in Figure 24, during simple harmonic vibration of plane electron, a cosinoidal power line has been formed as a result of distortion of electric field generated by the electron. The transmission of electric field is similar to the transmission of ripple on the surface of water. The electric field substances at all the points on the power line will be in the same motion at velocity $u$ along the direction of $\boldsymbol{Y}$ coordinate along with the electron early or late, which will not move along the direction of $\boldsymbol{X}$ coordinate. E represents the electric field intensity at any point $\mathrm{P}$ on the power line (along the tangential direction of Point $\mathrm{P}$ ); the component of $\mathrm{E}$ in the direction of $\boldsymbol{Y}$ coordinate: $\boldsymbol{E}_{y}=E \sin \alpha=-E_{0} \operatorname{tg} \alpha \quad$ [see (Formula 33)]; the component of $\mathrm{E}$ in the direction of $\boldsymbol{X}$ coordinate: $\boldsymbol{E}_{x}=-E \cos \alpha=-E_{0}$ [see (Formula 31)]. Obviously, $\boldsymbol{E}_{y}$ indicates the intensity of varying electric field transmitted via the electromagnetic wave. It is observed that, the real power line of electromagnetic wave is a complete Line $\boldsymbol{E}$ derived from the moving electron, while the varying electric field can be expressed by all the lines $\mathrm{E}_{\mathrm{y}}$ with a certain length, i.e. the calculated value of component of Line $\boldsymbol{E}$ along the direction of $Y$ coordinate, rather than the independent varying electric field.

Since the electric field intensity means that the electric field of $\boldsymbol{E}$ will move at velocity of $u$ along the direction of $\boldsymbol{Y}$ coordinate, so $u$ shall be far less than the velocity of light C, i.e. $\operatorname{tg} \alpha \rightarrow 0$, which can be considered as $u \perp E$, that is, E represents a moving electric field whose direction of motion is perpendicular to the 
direction of propagation ( $\mathrm{x}$ ), namely, a magnetic field; its magnetic induction intensity $\mathrm{B}$ can be calculated as per the Formula (37): $\boldsymbol{B}=\mu_{0} \varepsilon_{0} u E_{x}=-\mu_{0} \varepsilon_{0} u E_{0}$. Since $B$ is proportional to $u$, while $\boldsymbol{E}_{y}=-E_{0} \operatorname{tg} \alpha=-E_{0} u / C$ which is also proportional to $u$, so $\boldsymbol{B}$ shall be of the same phase position as $E_{y}$ at any time. Since the source of $\mathrm{B}$ is irrelevant to $E_{\mathrm{y}}$, and the source of $E_{\mathrm{y}}$ is unrelated to $\boldsymbol{B}$, the electromagnetic wave never means that the varying magnetic field $(\boldsymbol{B})$ is generated by the varying electric field $\left(E_{y}\right)$, or that varying electric field is generated by the varying magnetic field.

Since $W=B^{2} / 2 \mu_{0}, B=-\mu_{0} \varepsilon_{0} u E_{0}$, the energy of magnetic field of electromagnetic wave can be calculated according to the following formula:

$$
W=\frac{1}{2 \mu_{0}}\left(-\mu_{0} \varepsilon_{0} u E_{0}\right)^{2}=\frac{1}{2 \mu_{0}}\left(\frac{\mu_{0} \varepsilon_{0} u^{2} E_{0}^{2}}{C^{2}}\right)=\frac{\varepsilon_{0}}{2} E_{0}^{2} \frac{u^{2}}{C^{2}}=\frac{\varepsilon_{0}}{2}\left(E_{0} \operatorname{tg} \alpha\right)^{2}=\frac{\varepsilon_{0}}{2} E_{y}^{2}
$$

The above formula shows that, the energy of magnetic field of electromagnetic wave shall be identically equal to the energy of transmitted varying electric field $\left(E_{\mathrm{y}}\right)$.

\subsection{Electromagnetic Wave and Electromagnetic Induction Law}

As shown in Figure 25 (i.e. the simplified diagram of Figure 22), only the two lines E have been drawn; moreover, the distance $\boldsymbol{l}$ shall be changed as the micro distance $\Delta x$. The Formula 5.1 shows that, the law of motion of all electric field points at $\mathrm{P}$ along the direction of $\mathrm{y}$ coordinate shall be: $y_{1}=f(t)$; the law of motion of all electric field points at $\boldsymbol{l}$ behind Point $\mathrm{P}$ along the direction of y coordinate shall be: $y=f(t-l / c)$; therefore, the law of motion of all electric field points at the micro distance $\Delta x P^{\prime}$ behind Point $\mathrm{P}$ along the direction of y coordinate shall be: $y_{2}=f(t-\Delta x / c)$. The Formula (33) shows that, the component of $\mathrm{E}$ in the direction of y coordinate shall be: $\boldsymbol{E}_{v}=-E_{o} \operatorname{tg} \alpha \boldsymbol{Y}_{0}$.

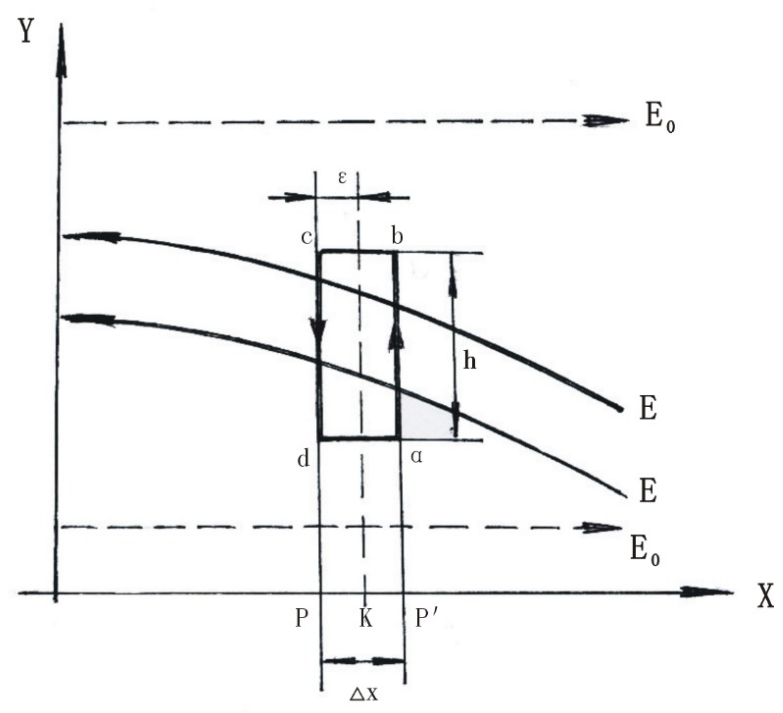

Figure 25. Potential on the closed loop generated by distortion of power line

It is indicated in the Formula 5.1 that, since $\cos \alpha$ is a negative value $(\alpha>\pi / 2)$, so $\operatorname{tg} \alpha$ shall also be a negative value. If $d x$ is taken as the normal increments, then:

$$
\operatorname{tg} \alpha=-\frac{d y}{d x}=-\frac{d f(t)}{d x} .
$$

After this formula is substituted into the Formula (33), the following formula can be obtained:

$$
\begin{gathered}
\boldsymbol{E}_{y_{1}}=E_{o} \frac{d f(t)}{d x} \boldsymbol{Y}_{0} \\
\text { and } \boldsymbol{E}_{y_{2}}=E_{o} \frac{d f(t-\Delta x / c)}{d x} \boldsymbol{Y}_{0}
\end{gathered}
$$


Obviously, $\boldsymbol{E}_{y_{1}}$ and $\boldsymbol{E}_{y_{2}}$ are the positive values. The Formula (43) \& (44) can be written as the scalar formula below:

$$
E_{y_{1}}=E_{o} \frac{d f(t)}{d x} \text { and } E_{y_{2}}=E_{o} \frac{d f(t-\Delta x / c)}{d x}
$$

The potential on the loop abcd along the right-handed helical direction shall be shown in Figure 3 (analysis diagram):

$$
\oint E d l=\int_{a}^{b} E_{y 2} d y+\int_{b}^{c}\left(E_{x}-E_{0}\right) d x+\int_{c}^{d}-E_{y 1} d y+\int_{a}^{d}\left(-E_{x}+E_{0}\right) d x
$$

Obviously, the above-mentioned item 4 and item 2 shall be equal in size of the integral, in which one positive and one negative can be offset each other, so that:

$$
\oint E d l=\int_{a}^{b} E_{y 2} d y-\int_{c}^{d} E_{y 1} d y
$$

Because of the same $E_{y 2}$ on the line ab and the same $E_{y 1}$ on the line cd, $h$ represents the length of ab and cd, so that the above formula can be converted into:

$$
\oint E d l=E_{y 2} h-E_{y 1} h
$$

After the $E_{y 1}$ and $E_{y 2}$ in Formula (43) and (44) are substituted into the above formula, the following formula can be obtained:

$$
\begin{aligned}
\oint E d l & =E_{o} h \frac{d}{d x} f(t-\Delta x / c)-E_{o} h \frac{d}{d x} f(t) \\
& =-E_{o} h \frac{d}{d x}[f(t)-f(t-\Delta x / c)]
\end{aligned}
$$

According to the Lagrangean mean value theorem, the following formula can be obtained:

$$
f(t)-f(t-\Delta x / c)=\left[t-\left(t-\frac{\Delta x}{c}\right)\right] \frac{d}{d t} f\left(t-\frac{\varepsilon}{c}\right)=\frac{\Delta x}{c} \frac{d}{d t} f\left(t-\frac{\varepsilon}{c}\right)
$$

In the above formula, $\mathcal{E}$ represents the micro distance behind $\mathrm{P}(\mathcal{\varepsilon}<\Delta x$, as shown in Figure 25, at Point $\mathrm{K})$. Since $d f\left(t-\frac{\varepsilon}{c}\right) / d t$ indicates the velocity of E along the direction of y coordinate at $\mathrm{K}$ [Formula (30)], supposing that $u$ represents this velocity, then the above formula can be converted into:

$$
f(t)-f(t-\Delta x / c)=\frac{\Delta x}{c} u
$$

After the above formula is substituted into the Formula (45), the following formula can be obtained:

$$
\begin{gathered}
\oint E d l=-E_{o} h \frac{d}{d x}\left(\frac{\Delta x}{c} u\right)=-E_{o} \frac{h \Delta x}{c} \frac{d u}{d x} \\
\text { Since } \frac{d u}{d x}=\frac{d u}{d t} \cdot \frac{d t}{d x}
\end{gathered}
$$

Also, since $d x / d t=C$ and $h \Delta x$ represents the area surrounded by the loop abcd (expressed in S), then:

$$
\oint E d l=-\frac{E_{o} S}{c^{2}} \frac{d u}{d t}=-\frac{d}{d t} S \cdot \frac{E_{o} u}{c^{2}}
$$

According to the Formula (37), when $u$ is perpendicular to $E_{o}$, since $B=E_{o} u / c^{2}$, the above formula can be converted into:

$$
\oint E d l=-\frac{d}{d t} B \cdot S
$$

Since B.S is the magnetic flux $\phi$ surrounded by the loop abcd (Loop C), so the above formula can be written as: 


$$
\oint E d l=-\frac{d}{d t} \phi
$$

Obviously, the Formula (46) is an expression for the law of electromagnetic induction.

\subsection{Analysis of the Law of Electromagnetic Induction}

A current I long rectangular coil is shown in Figure $26(a)$. The left or right side of coil may be regarded as a current board. If it is equipped with $\mathrm{n}$ pieces of guide lines per unit length, this current board equivalently has the surface current (surface current density: nI). For simple analysis of problems, we may take the two surface plates A \& B (height h) between two pieces of current boards for analysis. As shown in Figure 26 (b), A and B can represent the two pieces of current boards. The direction of motion of negative electrons of current Boards A \& B shall be opposite to I. As can be seen, the current Boards A \& B shall be similar to the "infinite" surface plates (as shown in Figure 22). When there are any changes in current size of coil, the negative electrons on current Boards A \& B will be in variable motion. Thus, based on the above analysis, the current Boards A \& B will launch the electromagnetic waves towards the left and right, respectively. According to the analytical derivation (Section 4), the electromagnetic waves launched by the current Boards A \& B towards the left and right shall be consistent with the results of Formula (46).

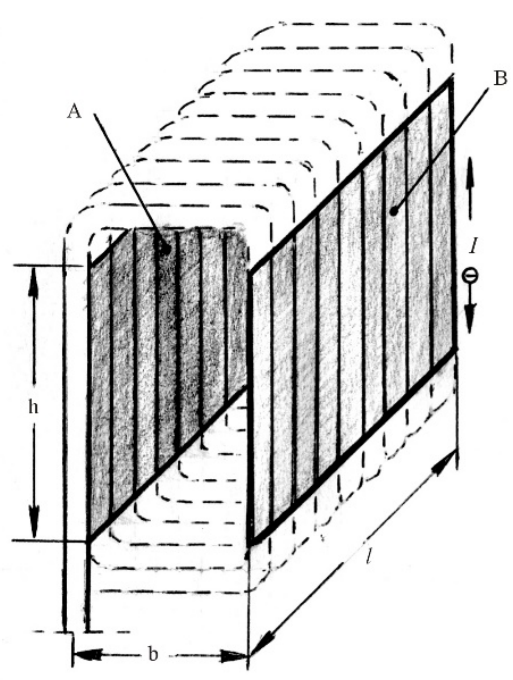

(a)

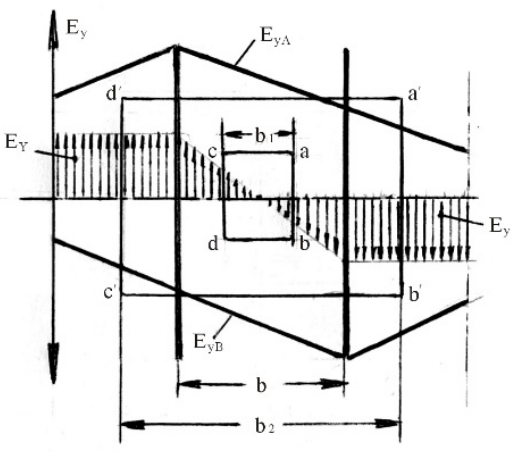

(b)

Figure 26. Distribution diagram of electric fields inside and outside the coil in case of current changes

We can analyze any changes in size of $E_{y}$ propagated by A \& B towards the left and right. According to the Formula (33) and (35), the scalar formula can be derived below:

$$
\begin{gathered}
E_{y}=E_{o} \frac{d y}{d x}=E_{o} \frac{u}{c} \\
\text { Obviously, } \frac{d}{d t} E_{y}=\frac{E_{o}}{c} \cdot \frac{d u}{d t}=\frac{E_{o}}{c} a
\end{gathered}
$$

In the Formula (48), $a$ represents the accelerated velocity of negative electron in variable motion. That is to say, any changes in size of $E_{y}$ shall depend on the accelerated velocity of negative electron in variable motion.

We can analyze the electric field near the coil, e.g. the electric field within $3 \mathrm{~m}$ from the coil. For the electromagnetic wave propagated at velocity of light, the time required for propagating the electromagnetic wave to $3 \mathrm{~m}$ from the coil shall be less than $10^{-8}$ seconds or so. Within such a short period of time, it can be considered that there is the same magnitude of motion acceleration $a$ of electric field near the current Boards A \& B ( $a$ is unchanged within the time $d t$ ), namely, $a$ is unchanged. If $a$ is unchanged, $E_{y}$ will be certainly increased or 
decreased in the proportion of $a$ along the direction of $x$ coordinate. If $a$ is set as a positive value, $E_{y}$ will be increased in the fixed proportion of $a$ after moving to the current Board A, so that we can draw the size height line of $E_{y}$ on the left and right of current Board A (see the two straight lines on the top of Figure $26(b)$ ). Since the direction of motion of negative electron on the current Board B is opposite to the direction of motion of negative electron on the current Board $\mathrm{A}$, so the size height line of $E_{y}$ on the current Board B is opposite to the size height line of $E_{y}$ on the current Board A (as shown in the Figure). The combination of $E_{y}$ inside and outside the current Boards A \& B shall refer to the combination of height lines of $E_{y}$ on the current Boards A \& B. The distribution diagram of combined $E_{y}$ shall be shown in Figure (b). It's easy to see from the distribution diagram that, inside the current Boards A \& B (coil), the potential on loop abcd shall be proportional to the width $b_{1}$ of loop, which is proportional to the area of loop or the magnetic flux surrounded by the loop. However, outside the current Boards A \& B (coil), the potential on loop $\mathrm{a}^{\prime} \mathrm{b}^{\prime} \mathrm{c}^{\prime} \mathrm{d}^{\prime}$ shall be unrelated to the width $b_{2}$ of loop, which is irrelevant to the area of loop; the potential on loop $\mathrm{a}^{\prime} \mathrm{b}^{\prime} \mathrm{c}^{\prime} \mathrm{d}^{\prime}$ shall be equivalent to an electric potential produced when the width of loop is the same as the width $b$ of coil; that is to say, the potential on loop a' $\mathrm{b}^{\prime} \mathrm{c}^{\prime} \mathrm{d}^{\prime}$ shall be only proportional to the magnetic flux in the coil. This explains why there is no any induced electric field existing in the magnetic field space outside the transformer.

\subsection{Analysis of Maxwell's Equations}

The Maxwell's equations consist of 4 equations below:

$$
\begin{aligned}
& \int_{S} \boldsymbol{D} \cdot d \boldsymbol{S}=Q \\
& \oint_{1} \boldsymbol{E} \cdot d \boldsymbol{l}=-d \phi / d t \\
& \oint_{\boldsymbol{H}} \cdot d \boldsymbol{l}=I+d \phi_{D} / d t \\
& \int_{S} \boldsymbol{B} \cdot d \boldsymbol{S}=O
\end{aligned}
$$

In the Formula ( $a), \boldsymbol{D}=\varepsilon \boldsymbol{E}$ represents the electric displacement vector, i.e. Gauss theorem. As described on the physical books, the Gauss theorem is derived from the Coulomb's law, (and not explained here). It should be noted that, the Formula $(a)$ is not generally true, because the experiments show that, there exists an electrostatic field around the permanent magnet (such a non-charged body), and the negative electrostatic field is greater than the positive electrostatic field, so that the electric displacement flux of closed surface surrounding the permanent magnet is not zero, so the Gauss theorem is not true. Also, the current-carrying coil can be proved by means of the inference method, namely, there exists an electrostatic field around the permanent magnet, so that there exists an electrostatic field around the current-carrying conductor. So, the Gauss theorem is not true as well. However, for analysis of electromagnetic wave, the electrostatic field around the permanent magnet or current-carrying coil can not be considered.

( $b$ ) (i.e. the law of electromagnetic induction) shall be described in Section 5.4\&5.5.

In the Formula $(c), \oint \boldsymbol{H} \cdot d \boldsymbol{l}=I+d \phi_{D} / d t$,

Called the law of total current, $\phi_{D}$ represents the electric displacement flux. The source of Formula $(c)$ shall be described as follows:

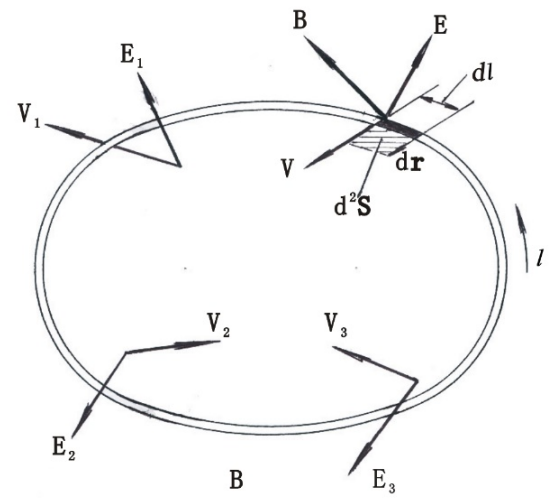

Figure 27: $d \phi_{D} / d t$ generated by the moving electric field when passing through Loop $l$ 
As shown in Figure 27, $l$ represents any closed loop; with the winding direction: of right-handed helical direction (counterclockwise direction). It can be assumed that there are a lot of electric fields with different sizes and directions as formed in electron motion around the loop, $\boldsymbol{E}_{1} \boldsymbol{V}_{1}, \boldsymbol{E}_{2} \boldsymbol{V}_{2}, \boldsymbol{E}_{3} \boldsymbol{V}_{3} \ldots \ldots$. We can analyze the motion of electric field at $d \boldsymbol{l}$ segment (with the same direction as $\boldsymbol{l}$ ) in terms of graphical representation.

The upward direction of electric field is defined as the positive $\boldsymbol{E}$. The positive $\boldsymbol{E}$ passing through $d \boldsymbol{l}$ from the right end to the left end is called the positive pass-through, because this pass-through will increase the quantity of positive electric field of loop $\boldsymbol{l}$. Similarly, the $-\boldsymbol{E}$ passing through $d \boldsymbol{l}$ from the left end to the right end is also called the positive pass-through, because this pass-through will reduce the quantity of positive electric field of loop $\boldsymbol{l}$. In contrast with the above two cases, it shall be called the negative pass-through.

As shown in Figure 27, the moving $\boldsymbol{E}$ passes through $d \boldsymbol{l}$ at velocity $\boldsymbol{V}$ within the time $d t$, and has moved forward to the distance $d \boldsymbol{r}$. $d \boldsymbol{l}$ and $d \boldsymbol{r}$ ( $\boldsymbol{r}$ and $\boldsymbol{V}$ are in the same direction) will form an elementary area $d \boldsymbol{l} \times d \boldsymbol{r}$ for the length of side, respectively. Since $d \boldsymbol{l}$ and $d \boldsymbol{r}$ are both infinitesimal, so the product shall be the second-order infinitesimal, in which the elementary area is expressed by $d^{2} \boldsymbol{S}=d \boldsymbol{l} \times d \boldsymbol{r}$. According to the Formula (1), E shall be in motion at $\boldsymbol{V}$, to generate $\boldsymbol{B}=\mu_{o} \varepsilon_{o} \boldsymbol{V} \times \boldsymbol{E}$, which can also be written as $\boldsymbol{H}=\boldsymbol{B} / \mu_{0}=\varepsilon_{o} \boldsymbol{V} \times \boldsymbol{E}=\boldsymbol{V} \times \boldsymbol{D}$. The magnetic potential generated by $\mathrm{H}$ on $d \boldsymbol{l}$ shall be $\boldsymbol{H} \cdot d \boldsymbol{l}=(\boldsymbol{V} \times \boldsymbol{D}) \cdot d \boldsymbol{l}$. According to the vector formula, $(\boldsymbol{V} \times \boldsymbol{D}) \cdot d \boldsymbol{l}=(d \boldsymbol{l} \times \boldsymbol{V}) \cdot \boldsymbol{D}$. Since $\boldsymbol{V}=d \boldsymbol{r} / d t$, this formula can be written as:

$$
\boldsymbol{H} \cdot d \boldsymbol{l}=\left(d \boldsymbol{l} \times \frac{d \boldsymbol{r}}{d t}\right) \cdot \boldsymbol{D}=\frac{1}{d t}(d \boldsymbol{l} \times d \boldsymbol{r}) \cdot \boldsymbol{D}=\frac{1}{d t} d^{2} \boldsymbol{S} \cdot \boldsymbol{D}
$$

In the above formula, $d^{2} \boldsymbol{S} \cdot \boldsymbol{D}$ represents the electric displacement flux of $\mathbf{D}$ in an elementary area $d^{2} \boldsymbol{S}$, expressed in $d^{2} \phi_{D}$. So, the above formula can be transformed into $\boldsymbol{H} \cdot d \boldsymbol{l}=d^{2} \phi_{D} / d t$. As a result, the following formula can be obtained:

$$
\oint \boldsymbol{H} \cdot a \boldsymbol{l}=\int_{s} d^{2} \phi_{D} / d t=d \phi_{D} / d t
$$

Obviously, in the Formula (49), $d^{2} \phi_{D} / d t$ represents the displacement current.

As mentioned above, the magnetic potential $\boldsymbol{H} \cdot d \boldsymbol{l}$ will be generated at any point on the loop $l$ as a result of moving electric field. After such an analysis, we have not only clarified the underlying causes for generation of magnetic potential, but also have pointed out the physical significance of displacement current. Of course, the polarization current also belongs to the displacement current. Since the polarization current is also part of electrons in the electric field, so the Formula (49) still holds.

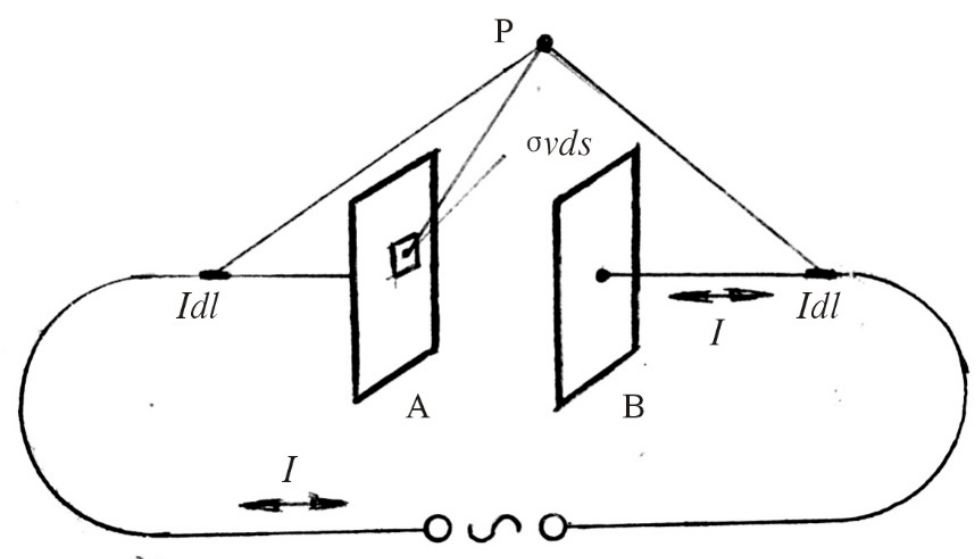

Figure 28. Biot-Savart Law and Displacement Current

Why did Maxwell put forward the displacement current? Introduction to the information: When analyzing the AC circuit (alternating current circuit) (Figure 28), Maxwell observed that there was a magnetic field at Point $\mathrm{P}$ between the two capacitor plates A and B; however, A and B are disconnected; there was no current between A and $\mathrm{B}$, but only the varying electric field. In order to make the current continuous, he assumed the varying electric field as the electric current - displacement current. Through the moving electric field, i.e. magnetic 
field $\left(B=\mu_{0} \varepsilon_{0} V \times E\right)$, the essence of displacement current has been described. In fact, if the displacement current is not applied, only on the basis of Biot-Savart law, we can also accurately obtain the magnetic field intensity at Point P. As shown in the figure, we can not only calculate the composed magnetic field intensity of each piece of $I d l$ on the return conductor at Point P, but also can calculate the composed magnetic field intensity of all surface current elements $\sigma v d s$ on the panels $\mathrm{AB}$ at Point P. The total magnetic field intensity is the actual magnetic field intensity at Point P. We will further analyze the relationship between the circuit magnetic potential and the current-carrying conductor as follows.

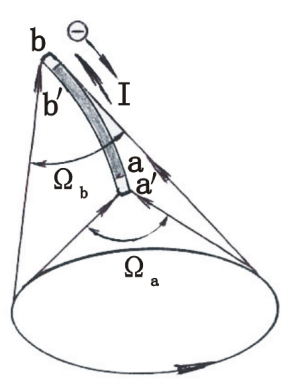

(a)

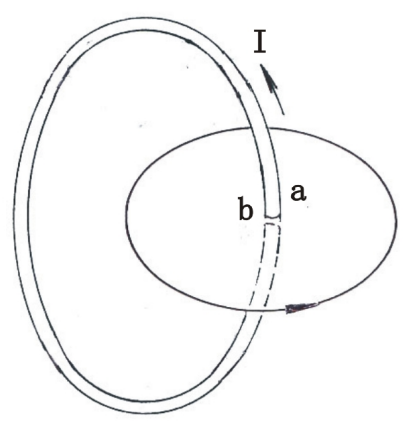

(b)

Figure 29: Proof of the Ampere Circuit Law

$a b$ represents a section of current wire (as shown in Figure $29(a)$ ); the direction of current I: From bottom to top; the actual direction of motion of free electron: From top to bottom; the winding direction of loop $l$ : Right-handed helical direction of I. Within the time $d t$, the negative electron beam will move from $b a$ to $b^{\prime} a^{\prime} . b a^{\prime}$ represents the overlap between the two negative electron beams $b a$ and $b^{\prime} a^{\prime}$ (black thick line in Figure 29). Clearly, within the time $d t$, there is the same electric displacement flux of loop $l$ generated by $b^{\prime} a$ the overlapped negative electron beam which is unrelated to any changes in the electric displacement flux in the loop $l$. Therefore, $b^{\prime} a^{\prime}$ is bb' less than $b a$, but aa' more than $b a$, resulting in any changes in the electric displacement flux of loop $l$; according to the law of conservation of matter, bb' and aa' shall be of the same electric quantity, set as $-d Q$. Clearly, within the time $d t$, the negative electron beam $b a$ is transformed into the negative electron beam $b^{\prime} a^{\prime}$. For any changes in the loop $l,-d Q$ of bb' is transformed into $-d Q$ of aa'. When deriving the relationship between the Gauss theorem and the Coulomb's law in physics, it is pointed out that, the electric displacement flux $d \phi_{D}$ and $d Q$ generated by $d Q$ in a certain area shall be proportional to the solid angle $\Omega$ subtended by such an area; relationship: $d \phi_{D}=\Omega d Q / 4 \pi$ (the solid angle of entire closed surface shall be: $4 \pi$ ). The solid angle of $-d Q$ of aa' subtended by $l: \Omega_{a}$; the solid angle of $-d Q$ of bb' subtended by $l: \Omega_{b}$; the upward direction of electric displacement generated by $-d Q$ of aa' and bb': Positive value. Thus, within the time $d t$, any changes in the total electric displacement flux of loop $l$ shall be as follows:

$$
\begin{aligned}
& \qquad d \phi_{D}=d \phi_{a a^{\prime}}-d \phi_{b b^{\prime}}=\frac{d Q}{4 \pi}\left(\Omega_{a}-\Omega_{b}\right) \\
& \text { The above formula can be transformed into: } \quad \frac{d \phi_{D}}{d t}=\frac{d Q}{d t} \frac{\left(\Omega_{a}-\Omega_{b}\right)}{4 \pi}=I\left(\frac{\Omega_{a}-\Omega_{(50)}}{4 \pi}(50)\right.
\end{aligned}
$$

The magnetic potential of loop $l$ can be calculated according to the Formula (50):

$$
\oint \boldsymbol{H} \cdot a \boldsymbol{l}=\frac{d \phi_{D}}{d t}=\frac{\left(\Omega_{a}-\Omega_{b}\right)}{4 \pi} I
$$

As shown in Figure 29 (b), the closed lead of Current I can pass through $l$. We can treat this closed lead as Point a on the surface consisting of loop $l$ to Point $\mathrm{b}$ under the surface. Then, in accordance with the analysis method in the Formula (51), it can be considered that, $\Omega_{a}=2 \pi, \Omega_{b}=-2 \pi$. Therefore, the magnetic potential on loop $l$ generated by the closed lead (as shown in Figure 29 (b)) can be calculated according to the Formula (51):

$$
\oint \boldsymbol{H} \cdot a \boldsymbol{l}=\frac{\left(\Omega_{a}-\Omega_{b}\right)}{4 \pi} I=\frac{2 \pi-(-2 \pi)}{4 \pi} I=I
$$


Obviously, the Formula (21) shall be considered as Ampere's circuital law. According to the Formula (51) \& (52), it can be deduced that, taking into account all the currents, the following formula can be obtained:

$$
\oint \boldsymbol{H} \cdot a \boldsymbol{l}=I+d \phi_{o} / d t
$$

This is the law of total current, i.e. the Formula (c) of Maxwell's equations. It can be seen that, the total current shall be attributed to the motion of charged particles in an electric field.

It should be noted that, the law of total current does not apply to some special cases. For example, a charge $d Q$ can pass through the center of circular loop surface of radius $r$ at velocity $\boldsymbol{V}$. When the electric charge is passing through the center in an instant, according to the Biot-Savart Law or $d \boldsymbol{B}=\mu_{o} \varepsilon_{o} \boldsymbol{V} \times d \boldsymbol{E}$, we can obtain $d \boldsymbol{H}=d Q \boldsymbol{V} / 4 \pi r^{2}$ at any point on the circular ring and the magnetic potential on the circular $\operatorname{ring} \oint \boldsymbol{H} \cdot d \boldsymbol{l}=d Q V / 2 r$. There is no doubt that this result is correct. However, according to the law of full current (Formula (c)), no matter how to calculate this formula, the correct result can not be obtained.

In the Formula $(\mathrm{d}), \int_{S} B \cdot d s=0$

It is known that, the magnetic field generated by any negative electron or positive electron (hereinafter generally referred to as the electron) in motion shall be determined in accordance with the Biot-Savart Law. It is known from the Biot-Savart Law that, the line B generated by the electron moving at velocity $\boldsymbol{V}$ shall be considered as a concentric circle by taking as the axis moving at velocity $\boldsymbol{V}$. Therefore, for any closed surface, if the line B in a certain concentric circle passes through this closed surface, then this line B will also pass back to this closed surface, namely, the pass-through shall be equivalent to the penetration; if the pass-through line $\mathrm{B}$ is zero, the total line B piercing the closed surface will also inevitably be zero. It can be deduced from this that, in the resultant magnetic field generated by $2,3 \ldots . . \mathrm{n}$ electrons moving, since the magnetic flux of each electron piercing the closed surface is zero, so the Formula (D) holds.

\subsection{The Actual Velocity of Light is the Ideal Velocity of Light, isn't it?}

As previously described, the electromagnetic wave is propagated at the velocity of light. If the electromagnetic wave is propagated at the faster or slower speed, what different effects will be produced?

As a result of analysis, the faster the propagation velocity of electromagnetic wave, i.e. the faster the velocity of light, the faster the propagation \& reception velocity of electromagnetic wave signal, the greater, more convenient and advantageous the astronomical observation. However, it's not good if the velocity of light is too fast, because of the signal intensity of electromagnetic wave propagation: $E_{v}=E_{0} u / c$ (as shown in the Formula (47)). At a certain motion velocity $u$ of wave-source negative electron, the larger c, the smaller $E_{y}$. The Formula $\mu_{0}=1 / \varepsilon_{0} c^{2}$ shows that, the strength of magnetic phenomenon shall be inversely proportional to $\mathrm{C}^{2}$. If $\mathrm{C}$ is infinitely great, naturally, there are no magnetism and electromagnetic wave. For example: If the velocity of light is increased from $3 \times 10^{8} \mathrm{~km}$ per second to $3 \times 10^{9} \mathrm{~km}$ per second, $\mathrm{C}^{2}$ will be increased by 100 times, while $\mu_{0}$ will be decreased by 100 times. If $\mu_{0}$ is too slow, the electromagnetic wave signal will become very weak. Of course, it's absolutely impossible to invent the radio communication model. Therefore, we don't want the velocity of light to become too fast. However, it is not good if the velocity of light is too slow. If the velocity of light is too slow, the signal propagation \& reception will become slow; $E_{y}$ and $\mu_{0}$ will be increased; the radiated electromagnetic interference will be greatly enhanced. This is very detrimental to humans, even unfit for human being or other creatures to survive (in severe cases). Therefore, we don't want the velocity of light to become too slow. Obviously, the actual velocity of light that we feel is the most favorable velocity of light. So, it can be importantly deducted that the most ideal velocity of light shall be up to $300,000 \mathrm{~km}$ per second.

\section{Conclusion}

Since the electromagnetics can not explain why there exists an electric field around the permanent magnet, it can be concluded that there are some defects in electromagnetics. This paper further analyzes the defects of electromagnetic principle and fallacies of theoretical derivation from fourteen aspects. It is noted that, the basic defect of electromagnetics is that there is no explanation of any inherent causes for electromagnetic induction, displacement current, Lorentz force and other surface phenomena. Moreover, there are any errors in the theoretical derivation of electromagnetics, e.g. the same direction of action and reaction, infinitely high kinematic velocity of magnetic field, etc. The electromagnetics can neither make a scientific explanation for deflection results of high-velocity moving charged particles in a magnetic field, nor can determine whether there is problem for the 
moving magnetic field, etc. In order to eliminate the defects of electromagnetics, it is necessary to find the fundamental factor caused by all electromagnetic phenomena. For this purpose, it is indicated from analysis that, all the electromagnetic phenomena will be inevitably accompanied by an electron motion; and the fundamental factor for all electromagnetic phenomena is resulted from the motion of electron in an electric field. Combined with the basic principles of physics, this paper proposes the seven rules, e.g. principle of equivalent electric field, length contraction of electric field in the direction of motion, and the like; analyzes the uniform motion and variable motion in an electric field on the basis of such rules; clarifies that the magnetic field is an electric field of motion contraction; explains that the interaction force of current-carrying conductor is derived from the relative motion of positive and negative electrons, resulting in the contraction increase to varying degrees; indicates that the Lorentz force is a general effect caused by two electric fields of different kinematic velocity and contraction condition; qualitatively and quantitatively deduces there exist the negative and positive electric fields at some locations around the cubic permanent magnet according to the distribution formula for contraction motion of moving charged particles in an electric field and the sawtooth current model of permanent magnet. Through the analysis of an electric field in variable motion, this paper explains that, when the electron is in variable motion, the electromagnetic wave will be propagated at the velocity of light from near to far in surrounding electric field, resulting in distortion of electric field; derives the energy output of electromagnetic wave based on the distortion and contraction of electric field in the direction of motion; indicates that the electric field energy output by electromagnetic wave is identically equal to the magnetic field energy. Also, through the analysis of distorted electric field, this paper derives the law of electromagnetic induction; clarifies the physical process of electromagnetic induction; derives the displacement current according to a relation between the electromagnetic field and the moving electric field; proves the law of total current; resolves the substance of Maxwell's equations; clarifies that the electromagnetic phenomena is rooted in the contraction of electric field in the direction of motion, which is derived from the natural law of constant velocity of light. Since the magnetic phenomenon is a basic element of modern equipment, the natural law of constant velocity of light shall be considered a key for which the humanity has started the modernization process. From the analysis of electromagnetic wave propagation velocity of light, it is indicated that, if the velocity of light is increased, the propagation effect of electromagnetic wave will be necessarily decreased rapidly; if the velocity of light is decreased, the electromagnetic interference and radiation will be inevitably rapidly increased. Therefore, it is considered that, the most ideal velocity of light shall be up to about $300,000 \mathrm{~km}$ per second.

\section{References}

Tsinghua University electrodynamics teaching and research group. (1978). Electrodynamics compiled by Tsinghua University electrodynamics teaching and research group. China Higher Education Press.

Wu, S. B. (1987). Basics of Theory of Relativity. Shaanxi Science \& Technology Press.

Yao, K. (2012). Question on some principles of electromagnetism. Applied Physics Research, 4(3), 115.

Yao, K. (2013). Set up invariable axiom of force equilibrium and solve problems about transformation of force and gravitational mass. Applied Physics Research, 5(1), 72.

Yao, K. (2014). Actual Time Dilation is Dependent on Absolute Velocity It is Impossible for Human Being to Have Time Travel. Applied Physics Research, 7(1), 25.

Yao, K. (2014). Explanation of Electromagnetic Wave and Induction by Variation of Electric Fluxline and the Deduction of Magnetism Rooted in Constancy of Light Velocity. Applied Physics Research, 6(3), 112.

Yao, K. (2015). A New Explanation of Deflection Results of Charged Particles in High-velocity Motion in Magnetic Field C Correction of Lorentz Force. Applied Physics Research, 7(5), 13.

Yao, K. (2016). Complement of Special Relativity and Limitation of General Relativity. Applied Physics Research, $8(1), 81$.

Yao, K., \& Wang, Z. (2012). Inferring the fact that static magnetic field exists along with electrostatic field and conducting experimental verification in accordance with the theory of relativity. Applied Physics Research, 4(1), 222.

\section{Copyrights}

Copyright for this article is retained by the author(s), with first publication rights granted to the journal.

This is an open-access article distributed under the terms and conditions of the Creative Commons Attribution license (http://creativecommons.org/licenses/by/4.0/). 